Claudia Hensberg

Eigennützige

Regierungen im

fiskalischen Wettbewerb

um Kapital 


\section{Claudia Hensberg}

\section{Eigennützige Regierungen im fiskalischen Wettbewerb um Kapital}

Die Bedeutung von Ausgaben für öffentliche Zwischenprodukte wird in Modellen zum zwischenstaatlichen Wettbewerb um Kapital kaum berücksichtigt. In diesem Buch wird daher der Schwerpunkt der Betrachtung auf den Ausgabenwettbewerb gelegt. Insbesondere wird gezeigt, dass die fiskalpolitischen Instrumente - Steuern und Ausgaben für öffentliche Zwischenprodukte - als strategische Variablen zur Attrahierung von Kapital substituierbar sind. Aus dieser Analyse wird ein Wert ermittelt, der das Niveau beider fiskalpolitischer Instrumente zusammengefasst beschreibt. Des Weiteren wird untersucht, inwiefern Ausgabenwettbewerb durch Steuerharmonisierungsvorschriften beeinflusst wird. Darüber hinaus werden grundlegende, modelltheoretische Fragen zum zwischenstaatlichen Wettbewerb um Kapital thematisiert.

Claudia Hensberg wurde 1975 in Wuppertal geboren. Von 1994 bis 1999 studierte sie Wirtschaftswissenschaft an der Bergischen Universität-Gesamthochschule Wuppertal. Von 2000 bis 2003 promovierte sie in Wirtschaftswissenschaft am Lehrstuhl für Volkswirtschaftslehre, insbesondere Finanzwissenschaft, der Bergischen Universität Wuppertal. 
Eigennützige Regierungen im fiskalischen Wettbewerb um Kapital 


\section{FINANZWISSENSCHAFTLICHE SCHRIFTEN}

Herausgegeben von den Professoren

Konrad, Krause-Junk, Littmann, Oberhauser, Pohmer, Schmidt

Band 109

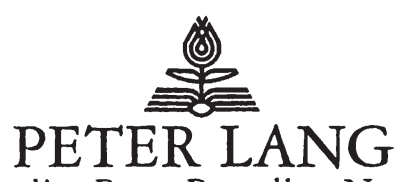

Frankfurt am Main - Berlin · Bern - Bruxelles - New York • Oxford · Wien 


\section{Claudia Hensberg}

\section{Eigennützige Regierungen im fiskalischen Wettbewerb um Kapital}

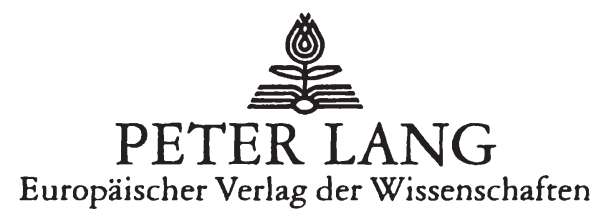

Claudia Hensberg - 978-3-631-75213-5 
Bibliografische Information Der Deutschen Bibliothek

Die Deutsche Bibliothek verzeichnet diese Publikation in der Deutschen Nationalbibliografie; detaillierte bibliografische Daten sind im Internet über <http://dnb.ddb.de > abrufbar.

Open Access: The online version of this publication is published on www.peterlang.com and www.econstor.eu under the international Creative Commons License CC-BY 4.0. Learn more on how you can use and share this work: http://creativecommons. org/licenses/by/4.0.

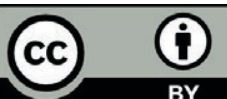

This book is available Open Access thanks to the kind support of ZBW - Leibniz-Informationszentrum Wirtschaft.

Zugl.: Wuppertal, Univ., Diss., 2003

Gedruckt auf alterungsbeständigem, säurefreiem Papier.

\author{
D468 \\ ISSN 0170-8252 \\ ISBN3-631-51244-9 \\ ISBN 978-3-631-75213-5 (eBook) \\ (C) Peter Lang $\mathrm{GmbH}$ \\ Europäischer Verlag der Wissenschaften \\ Frankfurt am Main 2003 \\ Alle Rechte vorbehalten.
}

Das Werk einschließlich aller seiner Teile ist urheberrechtlich geschützt. Jede Verwertung außerhalb der engen Grenzen des

Urheberrechtsgesetzes ist ohne Zustimmung des Verlages unzulässig und strafbar. Das gilt insbesondere für

Vervielfältigungen, Übersetzungen, Mikroverfilmungen und die Einspeicherung und Verarbeitung in elektronischen Systemen.

Printed in Germany 123457

www.peterlang.de 


\section{Vorwort}

Bei dem vorliegenden Beitrag handelt es sich um eine von der Bergischen Universität Wuppertal angenommene Dissertation. Sie befasst sich mit zentralen Aspekten des zwischenstaatlichen Wettbewerbs um Kapital zwischen eigennützigen Regierungen. In Anlehnung an die Literatur zum fiskalischen Standortwettbewerb wird in der Arbeit der Kapitalbestand in der betrachteten Föderation und der Schuldenstand als exogen vorgegeben und fix angenommen.

Betrachtet man die Realität, so ist selbstverständlich zwischen dem Realkapitalbestand und dem Kapitalzuwachs zu unterscheiden. Das Ausmaß des Kapitalzuwachses in einer Region wird durch die Attraktivität des Standortes und somit u.a. durch die Steuerpolitik und die Ausgabenpolitik bezüglich öffentlicher Zwischenprodukte beeinflusst. Kapitalwanderungen, derart dass Kapitalbestände in einer Region abgebaut und in einer anderen Region wieder aufgebaut werden, sind nur in Ausnahmefällen anzutreffen. Insofern wirkt die Annahme eines exogen vorgegebenen Kapitalbestandes, der bedingt durch fiskalpolitische Veränderungen zwischen den Regionen wandert, zunächst problematisch. Man kann sie allerdings dahingehend interpretieren, dass sie in stark vereinfachter Weise den Zusammenhang darstellt, dass Investoren bei sinkender Standortattraktivität Ersatzinvestitionen in dieser Region unterlassen und stattdessen Investitionen in Konkurrenzregionen vornehmen. Letztlich führt dieses dann bedingt durch die Abschreibungen zu einer geringeren Menge an Kapital in der Region selbst und zu einer höheren Kapitalmenge in den Konkurrenzregionen. Vereinfachend wird in diesem Sinne in der Arbeit von einer Kapitalwanderung gesprochen wohlwissend, dass die dahinterstehenden Zusammenhänge weitaus komplexer sind.

Darüber hinaus sollte auch bedacht werden, dass durch das verwendete Kapitalmarktmodell kein Kapitalzuwachs in der gesamten Föderation modelliert wird. In der Realität können wir jedoch einen mehr oder weniger stark wachsenden Kapitalbestand beobachten. Dieses Phänomen fließt in die Untersuchungen der Arbeit nicht ein, da das Ausmaß des Wachstums des Kapitalstocks in der Föderation als Ganzes für Fragestellungen des fiskalischen Standortwettbewerbs eher von untergeordneter Bedeutung ist. Um die Untersuchungen möglichst einfach zu halten, wird daher dieser Themenkomplex ausgeklammert, indem ein exogen vorgegebener Kapitalbestand angenommen wird. 
Wie erwähnt wird in dieser Arbeit die Problematik der Kreditfinanzierung öffentlicher Güter nicht explizit betrachtet. Daher wird in den Modellierungen von Kapitel 2 und 3 auch auf die Berücksichtigung einer Budgetgleichung verzichtet. In diesen Kapiteln steht die Wirkung von Steuern und öffentlichen Zwischenprodukten im zwischenstaatlichen Wettbewerb um Kapital im Mittelpunkt der Betrachtung. Eine Integration staatlicher Kreditfinanzierung in die Analyse würde die erzielten Ergebnisse in keiner Weise verändern, würde jedoch eine interessante Modellerweiterung darstellen.

Um Ausgabenwettbewerb zwischen eigennützigen Regierungen zu modellieren, wird in Kapitel 4 eine Leviathanregierung als erste Annäherung an ein adäquates entscheidungstheoretisches Modell einer eigennützigen Regierung gewählt. Diese Modellierung wird vorgenommen, um unabhängig von Steuerwirkungen die direkte Anreizwirkung des Ausgabenwettbewerbs auf eigennützige Regierungen zu erfassen. Als Einnahmemöglichkeit des Staates zur Erzielung seines Finanzierungsüberschusses wird lediglich eine Kapitalsteuer zugelassen. In der Realität steht dem Staat neben der Möglichkeit, Steuern zu erheben, jedoch auch die Möglichkeit der Verschuldung offen. Eine Integration von Staatsverschuldung in das Modell würde die Substanz der erhaltenen Ergebnisse nicht wesentlich verändern, würde allerdings die Darstellung stark verkomplizieren. Daher wird von einer Betrachtung der Staatsverschuldung Abstand genommen.

An dieser Stelle möchte ich mich bei all denjenigen bedanken, die mich bei der Anfertigung der vorliegenden Dissertation unterstützt haben. Mein besonderer Dank gilt meinem Betreuer Prof. Dr. Otto Roloff, dessen stete Diskussionsbereitschaft und interessanten Anregungen zum Gelingen dieser Arbeit beitrugen. Nicht zuletzt ermöglichte erst seine uneingeschränkte Unterstützung und die auBerordentlich gute, produktive Arbeitsatmosphäre eine Fertigstellung der Arbeit in der kurzen Zeit von 3 Jahren. Des Weiteren möchte ich mich bei Dr. Michael Pickhardt bedanken, der mich bei zahlreichen Kaffees in die Geheimnisse der wissenschaftlichen Forschungskultur einweihte. Die Möglichkeit, meine Forschungsergebnisse auf zahlreichen internationalen Tagungen zu diskutieren, ist nicht zuletzt auf den Erfahrungsaustausch mit ihm und Herrn Roloff zurückzuführen. Darüber hinaus gilt mein Dank aber auch allen übrigen Mitgliedern des Forschungsteams, Dr. Claudia Wesselbaum-Neugebauer und Dipl.-Vw. Cornelia Tausch, für die zahlreichen fruchtbaren Diskussionen. Zur Entwicklung meiner 
Arbeit hat die stets offene und konstruktive Kritik des Forschungsteams maßgeblich beigetragen. Im Weiteren möchte ich mich bei Prof. Dr. Bernd Rahmann für die Übernahme des Zweitgutachtens sowie bei den Prüfungskommissionsmitgliedern Prof. Dr. H.-J. Niessen, AOR Dr. W. von Wedel-Parlow und PD Dr. H. Frambach herzlich bedanken. Ich möchte schließlich auch Markus Kralik von Meyerswalden für die Hilfe bei der Formatierung der Arbeit zu einem publikationsreifen Manuskript danken.

Von besonderer Bedeutung war für mich die Unterstützung durch meinen Ehemann, Ender Aysal. Er stand mir stets bei allen Hochs und Tiefs hilfreich zur Seite. Mein Dank gilt auch meinen Eltern, die mir emotionale Unterstützung gaben. Meiner Schwester und meinen Freunden möchte ich dafür danken, dass sie - wenn nötig - ein offenes Ohr für mich fanden. 
Claudia Hensberg - 978-3-631-75213-5

Downloaded from PubFactory at 01/11/2019 07:02:00AM

via free access 


\section{Inhaltsverzeichnis}

Vorwort

Verzeichnis der Abbildungen

Verzeichnis der Symbole

1. Einleitung

1.1 Die Kontroverse zum Standortwettbewerb in der Literatur

1.1.1 Effizienzgewinn durch Standortwettbewerb

1.1.2 Effizienzverlust durch Standortwettbewerb

$1.2 \quad$ Problemstellung der Arbeit

1.2.1 Exogener Kapitalbestand oder vollkommen preiselastisches Kapitalangebot

1.2.2 Die Rolle der öffentlichen Güter

1.2.3 Formen eigennütziger Regierungen

1.3 Gliederung der Arbeit

2. Modellierung des Kapitalmarktes im Steuerwettbewerb

2.1 Vollkommen preiselastisches Kapitalangebot

2.1.1 Kapitalmarktgleichgewicht

2.1.2 Wirkung einer Steuersatzänderung

2.2 Exogen vorgegebener Kapitalbestand

2.2.1 Gleichgewicht auf dem Kapitalmarkt

2.2.2 Auswirkung einer Änderung des Steuersatzes 
3. Wirkung der fiskalpolitischen Instrumente auf dem

Kapitalmarkt im Wettbewerb

3.1 Grundmodell des fiskalischen Standortwettbewerbs

3.1.1 Fiskalpolitische Instrumente

3.1.2 Der Kapitalmarkt

3.2 Wirkung der einzelnen fiskalpolitischen Instrumente

$\begin{array}{ll}3.2 .1 & \\ 3.2 .2 & \end{array}$

3.2.2 Wirkung des öffentlichen Zwischenprodukts

3.3 Gesamtwirkung der fiskalpolitischen Instrumente einer Regierung

3.3.1 Substituierbarkeit fiskalpolitischer Instrumente

3.3.2 Fiskalpolitischer Instrumenten-Index 47

3.3.2.1 Wirkung eines fiskalpolitischen Instruments auf den 48

3.3.2.2 Gesamtwirkung der fiskalpolitischen Instrumente auf den Instrumenten-Index

3.4 Wettbewerb um Kapital 56

3.4.1 Wirkung des fiskalpolitischen Instrumenten-Indexes 56

3.4.2 Wettbewerb der Regionen $\quad 59$

$\begin{array}{ll}3.4 .3 & \text { Ruinöse Wettbewerbsspirale }\end{array}$

3.5 Zusammenfassung

4. Wirkung der Steuerharmonisierung auf

Leviathanregierungen im Standortwettbewerb

$4.1 \quad$ Das Modell

4.1.1 Die Regierung als Leviathan

4.1.2 Die Regierungen im nicht-kooperativen Nash-Spiel 
4.2 Das Surplusmaximum der Regierungen

4.3 Das Nash-Gleichgewicht

4.4 Die Bedeutung des harmonisierten Steuersatzes

4.5 Zusammenfassung

5. Fazit und Ausblick

Anhang 1 (Abschnitt a und Abschnitt b) zu Kapitel 2.2.2

Anhang 2 (Abschnitt a und Abschnitt b) zu Kapitel 2.2.2

Anhang 3 (Abschnitt a und Abschnitt b) zu Kapitel 2.2.2

Anhang 4 (Abschnitt a bis Abschnitt c) zu Kapitel 3.2.1

Anhang 5 (Abschnitt a bis Abschnitt c) zu Kapitel 3.2.2

Anhang 6 zu Kapitel 3.3.1

Anhang 7 (Abschnitt a bis Abschnitt c) zu Kapitel 3.4.1

Anhang 8 zu Kapitel 4.2

Anhang 9 zu Kapitel 4.2

Anhang 10 zu Kapitel 4.3

Anhang $11 \mathrm{zu}$ Kapitel 4.4

Literatur 


\section{Verzeichnis der Abbildungen}

Abbildung 1: Anpassung des Kapitalmarkts eines kleinen Landes 23

Abbildung 2: $\quad$ Anpassungen des Kapitalmarkts an eine Steuersatzände- 29 rung

Abbildung 3: Kapitalnachfragefunktion der i-ten Region 37

Abbildung 4: Gleichgewicht auf dem Kapitalmarkt 38

Abbildung 5: Wirkung einer Steuererhöhung in Region a 41

Abbildung 6: Wirkung einer Erhöhung der Bereitstellungsmenge des 44

Abbildung 7: Substitutionsfunktion $\quad 46$

Abbildung 8: Kurvenschar der Substitutionsfunktionen 47

Abbildung 9: Wirkung einer Änderung des Steuersatzes auf den In- 49 strumenten-Index

Abbildung 10: Wirkung der Bereitstellungsmenge an öffentlichem Zwi- 49 schenprodukt auf den Instrumenten-Index

Abbildung 11: Wirkung einer „Senkungspolitik“ in einer Hochsteuerre- 51 gion

Abbildung 12: „Senkungspolitik“ bei defizitärem Budget in einer Nied- 52 rigsteuerregion

Abbildung 13: „Senkungspolitik“ bei ausgeglichenem Budget in einer Niedrigsteuerregion

Abbildung 14: Erhöhungspolitik" bei defizitärem Budget in einer Hoch- 54 steuerregion

Abbildung 15: „Erhöhungspolitik“ bei ausgeglichenem Budget in einer Hochsteuerregion

Abbildung 16: Wirkung einer „Erhöhungspolitik“ in einer Niedrigsteuerregion

Abbildung 17: Wirkung einer Erhöhung des fiskalpolitischen Instrumenten-Indexes von Region a

Abbildung 18: Wettbewerb beider Regionen um Kapital

Abbildung 19: Wettbewerbsspirale mit permanent steigendem Nettozinssatz

Abbildung 20: Die Surplusfunktion einer Leviathanregierung

Abbildung 21: Lokales Maximum einer Surplusfunktion 
Abbildung 22: Globales Maximum einer Surplusfunktion bei $\mathrm{G}_{\mathrm{i}}=0 \quad 75$

Abbildung 23: Die Wirkung des harmonisierten Steuersatzes

80 


\section{Verzeichnis der Symbole}

a eine Region

b eine weitere Region

C Bereitstellungskosten des öffentlichen Zwischenprodukts

E Effizienzparameter

f Produktionsfunktion

G öffentliches Zwischenprodukt

i eine Region

I fiskalpolitischer Instrumenten-Index

j eine Konkurrenzregion

K Kapital

$\bar{K} \quad$ exogen vorgegebener Kapitalbestand

$\mathrm{K}_{\mathrm{d}} \quad$ Kapitalnachfrage

$\mathrm{K}_{\mathrm{s}} \quad$ Kapitalangebot

L Arbeit

n Zahl der Regionen

$\Pi \quad$ Gewinn

r Nettozinssatz

$\bar{r} \quad$ exogen vorgegebener Weltmarktzinssatz

$\mathrm{S} \quad$ Finanzierungsüberschuss

$\mathrm{t} \quad$ Steuersatz

$\tilde{t} \quad$ harmonisierter Steuersatz

T Steuereinnahmen

$\Phi \quad$ Technologiefunktion

w Bruttolohnsatz

Y Einheitsgut

z Bruttozinssatz 


\section{Einleitung}

Wir befinden uns in einer Zeit der Globalisierung, in der die Mobilität von Kapital und Menschen zunimmt. ${ }^{1}$ Aus der Sicht der einzelnen Regierungen bedeutet dies jedoch schlechthin, dass die Besteuerungsbasis insbesondere für Unternehmenserträge mobiler wird und sich somit ihrer Besteuerungshoheit entziehen kann. ${ }^{2}$ Der Wettbewerb der Regierungen um diese mobilen Faktoren wird daher kontinuierlich verschärft und findet beispielsweise in den stetig sinkenden nominellen Körperschaftsteuersätzen in Europa und der OECD seinen Ausdruck. ${ }^{3}$ Im Hinblick auf diesen feststellbaren Steuerwettbewerb wird auf die Erosion der nationalstaatlichen Steuersouveränität und auf mögliche Effizienzverluste hingewiesen. Um diesen nachteiligen Konsequenzen zu begegnen, wird die Harmonisierung der Steuern gefordert. ${ }^{4}$ Dem Wunsch nach Harmonisierung der Steuern wird jedoch entgegengehalten, dass gerade durch Standortwettbewerb die Effizienz verbessert werden kann. ${ }^{5}$ So wird die Diskussion über die Änderung des deutschen Finanzausgleichsgesetzes von der Vorstellung beherrscht, dass eine geringere Angleichung der Ländereinnahmen und somit ein stärkerer Wettbewerb zwischen den Gebietskörperschaften die Wohlfahrt steigert. ${ }^{6}$ Diesbezüglich stellt sich also immer wieder die Frage, wie viel Konkurrenz zwischen den einzelnen Regionen die allgemeine Wohlfahrt fördert und ab wann es sinnvoll wird, durch Harmonisierung von Steuern, durch einen Finanzausgleich oder durch die Koordination von Fiskalpolitik eventuell auftretenden, schädlichen Tendenzen entgegenzuwirken. ${ }^{7}$

\footnotetext{
${ }^{1}$ Vgl. Sinn (1990, S. 489), Edwards / Keen (1996, S. 113), Fuest (1997, S. 174; 2000, S. 357), Kollintzas / Philippopoulos / Vassilatos (2000, S. 1).

${ }^{2}$ Vgl. Edwards / Keen (1996, S. 113), Hange / Wellisch (1998, S. 315).

${ }^{3}$ Vgl. Edwards / Keen (1996, S. 113), Fuest (1997, S. 174), Sinn (1997, S. 13), Hange / Wellisch (1998, S. 315), Hensberg (2000, S. 13 ff.).

${ }^{4} \mathrm{Vgl}$. Edwards / Keen (1996, S. 114).

${ }^{5}$ Vgl. Frey / Eichenberger (1996, S. 337).

${ }^{6}$ Vgl. Peffekoven (1994, S. 297), Ebert / Meyer (1999, S. 106).

${ }^{7}$ Vgl. Buchanan / Lee (1994, S. 219), Edwards / Keen (1996, S. 114), Roloff (1999, S. 53 f.).
} 


\subsection{Die Kontroverse zum Standortwettbewerb in der Literatur ${ }^{1}$}

In der Literatur zum Steuer- und Standortwettbewerb existiert bisher keine einhellige Meinung, ob Standortwettbewerb unter dem Gesichtspunkt der Effizienz vorzuziehen oder abzulehnen ist. Befürworter des Standortwettbewerbs führen die durch ihn bedingten Effizienzgewinne an. Sie argumentieren, dass sich durch Standortwettbewerb die Präferenzen der mobilen Bürger offenbaren lassen und somit eine effiziente Versorgung mit öffentlichen Gütern sichergestellt werden kann. Als weiterer Vorteil wird die Möglichkeit der Berücksichtigung regional unterschiedlicher Präferenzen durch fiskalischen Föderalismus genannt. Darüber hinaus wird dem Standortwettbewerb die Fähigkeit zugeschrieben, die Besteuerungsmacht der Regierungen zu beschränken.

Demgegenüber heben Kritiker des Standortwettbewerbs insbesondere die durch ihn verursachten Effizienzverluste hervor. So kann zum einen aus einem zu starken Druck des Steuerwettbewerbs und zum anderen aus Nutzenspillovers eine Unterversorgung mit öffentlichen Zwischenprodukten resultieren. Bei ungleichen Regionen ist damit zu rechnen, dass eine Region auf Kosten einer anderen Region vom Wettbewerb profitiert.

\subsubsection{Effizienzgewinn durch Standortwettbewerb}

Die Diskussion in der Fiskalpolitik und der Literatur bezüglich Steuerwettbewerb und fiskalischem Wettbewerb wird von der Vorstellung geleitet, dass fiskalischer Wettbewerb zwischen Regierungen die Wohlfahrt der Bürger erhöht. ${ }^{2}$ Diese Vorstellung geht auf den Aufsatz „A Pure Theory of Local Expenditures“ von Charles M. Tiebout zurück, der bereits 1956 veröffentlicht wurde und besonders viel Beachtung seit Beginn der achtziger Jahre fand. ${ }^{3}$ In diesem Aufsatz

\footnotetext{
1 Dieser Überblick erhebt keinen Anspruch auf Vollständigkeit, sondern soll exemplarisch zentrale Aspekte in der Literatur zum Steuer- und Standortwettbewerb beleuchten. Er bezieht sich lediglich auf den Teil der Literatur, der sich mit der Wirkung von Standortwettbewerb auf die Allokation von Gütern und Produktionsfaktoren befasst. Für einen Überblick über die Wirkung von Standortwettbewerb bezüglich der Distribution und Stabilisierung siehe Wellisch (2000, S. 19 ff.).

${ }^{2}$ Vgl. Sinn (1997, S. 9 f.), Apolte (2001, S. 359).

${ }^{3} \mathrm{Vgl}$. Apolte (2001, S. 359 f.).
} 
legte Tiebout mit Hilfe eines durch starke Annahmen ${ }^{1}$ geprägten Modells dar, dass eine von dezentralen Regierungen vorgenommene Bereitstellung von öffentlichen Gütern sowie das entsprechende Steuersystem effizient sind. ${ }^{2}$ Mobile Haushalte wählen demnach die Regionen, durch die ihre Präferenzen für öffentliche Güter und die entsprechende Steuerbelastung am Besten erfüllt werden und offenbaren auf diese Art und Weise ihre Präferenzen bezüglich der Fiskalpolitik. ${ }^{3}$ Dieses Prinzip ist als „voting with one's feet“ bekannt geworden. ${ }^{4}$ Im Ergebnis führt es letztlich dazu, dass sich die Bereitstellungsmengen an öffentlichen Gütern sowie die dafür erhobenen Steuern von Jurisdiktion zu Jurisdiktion unterscheiden. ${ }^{5}$ Da die Bürger in die Jurisdiktionen ziehen, die ihren Präferenzen am besten gerecht werden, entstehen letztlich Jurisdiktionen, in denen Bürger mit relativ homogenen Präferenzen leben. ${ }^{6}$ Es liegt dann fiskalische Äquivalenz in dem Sinne vor, dass diejenigen, die Präferenzen für ein öffentliches Gut haben, dieses öffentliche Gut lokal zur Verfügung gestellt bekommen und die Finanzierungslast im gleichen Ausmaß tragen. ${ }^{7}$

Razin und Sadka (1991) gelangen mit Hilfe eines Modells mit kleinen Regionen und endogenem Arbeits- und Kapitalangebot zu ähnlichen Ergebnissen wie Tiebout. Sie schlussfolgern, dass das symmetrische Nash-Gleichgewicht effizient ist, wenn den Regierungen Kapital- und Lohnsteuern entsprechend dem Wohnsitzlandprinzip zugänglich sind. ${ }^{8}$ Eggert und Haufler (1999) zeigen, dass die Produktionseffizienz gewahrt bleibt, wenn identische Regierungen Zugriff auf eine Lohnsteuer und eine Kapitalsteuer nach dem Wohnsitzlandprinzip haben und die Angebotselastizität des Kapitals endlich ist. Myers (1990) belegt Tiebouts These mit Hilfe eines Modells mit großen, sich strategisch verhaltenden

I Vgl. Tiebout (1956, S. 417 ff.). Siehe für die Kritik dieser Annahmen Bewley (1981), Blankart (2001, S. 563 ff.).

${ }^{2}$ Vgl. Tiebout (1956), Bewley (1981, S. 713 f.), Oates / Schwab (1988, S. 333), Mieszkowski / Zodrow (1989, S. 1098), Sinn (1997, S. 9), Wrede (1998, S. 316), Apolte (2001, S. 359).

${ }^{3}$ Vgl. Tiebout (1956, S. 420), Boskin (1973, S. 204), Bewley (1981, S. 713), Richter (1994, S. 323), Hange / Wellisch (1998, S. 325), Wrede (1998, S. 316), Nowotny (1999, S. 404), Wellisch (2000, S. 15).

${ }^{4}$ Vgl. Oates / Schwab (1988, S. 333), Wildavsky (1990, S. 49), Sinn (1992, S. 179), Wrede (1998, S. 316), Wellisch (2000, S. 15).

3 Vgl. Tiebout (1956, S. 420), Hange / Wellisch (1998, S. 325), Brueckner (1999, S. 205), Nowotny (1999, S. 404).

${ }^{6}$ Vgl. Tiebout (1956, S. 420), Hange / Wellisch (1998, S. 325), Brueckner (1999, S. 205), Nowotny (1999, S. 404).

${ }^{7}$ Vgl. Graf (1999, S. 285).

${ }^{8}$ Vgl. Razin / Sadka (1991, S. 69 und S. 75), Eggert (1999, S. 1). 
Regionen, notwendigen fiskalpolitischen Instrumenten (insbesondere interregionalen Transferzahlungen) und mobiler Bevölkerung. Er legt mit Hilfe seines Modells dar, dass das Nash-Gleichgewicht von konkurrierenden Regionen Pareto-optimal ist. ${ }^{\prime}$

Tiebouts Tradition folgend sehen Oates (1972, 1977 und 1990), Boadway und Wildasin (1984) und Wellisch (2000) den Vorteil des fiskalischen Föderalismus in der Berücksichtigung von regional unterschiedlichen Präferenzen. Demnach ist die Verbindung einer regionalen Regierung zu ihren Bürgern enger als dies bei überregionalen Regierungen der Fall ist. ${ }^{2}$ Zentralregierungen tendieren dagegen dazu, die selbe Menge an öffentlichen Gütern in allen Regionen bereitzustellen, wodurch Effizienzverluste auftreten. ${ }^{3}$ Regionale Regierungen hingegen können diese von Region zu Region verschiedenen Präferenzen berücksichtigen. ${ }^{4}$ Insofern ist es effizienter oder wenigstens gleich effizient, wenn lokale Regierungen eine an die jeweilige Nachfrage angepasste Menge an öffentlichen Gütern anbieten, als wenn die Zentralregierung eine einheitliche Menge an öffentlichen Gütern für alle Regionen bereitstellt. ${ }^{5}$ Diese Aussage ist in der Finanzwissenschaft als Dezentralisierungstheorem bekannt geworden. ${ }^{6}$

Ein weiterer Vorteil von zwischenstaatlichem Wettbewerb wird in der Möglichkeit gesehen, die Bürger vor den Auswirkungen durch das Steuermonopol des Staates zu schützen. Im Gegensatz zu den zuvor genannten Argumenten, denen stets die Annahme einer wohlwollenden Regierung zugrunde liegt, wird dieses Argument von Autoren vertreten, die eigennützige Regierungen unterstellen. ${ }^{7}$ Dieser Ansicht nach profitieren die Bürger von dem Wettbewerb zwischen den Staaten in der selben Art und Weise wie dies die Konsumenten auf den Märkten für private Güter tun. ${ }^{8}$ Der Wettbewerbsdruck erhöhe demzufolge die Effizienz bezüglich politischer Entscheidungen und schütze den Bürger vor übermäßiger

\footnotetext{
' Vgl. Myers (1990, S. 116).

${ }^{2}$ Vgl. Oates (1990, S. 45 f.), Brümmerhoff (1996, S. 490), Wellisch (2000, S. 14).

${ }^{3}$ Vgl. Oates (1972, S. 11; 1977, S. 5 f.; 1990, S. 45), Boadway / Wildasin (1984, S. 500), Wellisch (2000, S. 15).

${ }^{4}$ Vgl. Oates (1972, S. 11 f.; 1977, S. 6; 1990, S. 45 f.), Boadway / Wildasin (1984, S. 499 f.), Wellisch (2000, S. 14).

${ }^{5} \mathrm{Vgl}$. Oates (1972, S. 11 ff.), Blankart (2001, S. 549 f.).

${ }^{6}$ Vgl. Blankart (2001, S. 549).

${ }^{7}$ Vgl. Brennan / Buchanan (1980, S. 171 f.), Buchanan / Lee (1994, S. 221), Wrede (1998, S. 316).

${ }^{8}$ Vgl. McLure (1986, S. 344 f.), Oates / Schwab (1988, S. 333). 
Besteuerung. ' Der zwischenstaatliche Wettbewerb wird daher als ein Instrument aufgefasst, um den Einfluss des öffentlichen Sektors in einer Volkswirtschaft zu beschränken. $^{2}$

\subsubsection{Effizienzverlust durch Standortwettbewerb}

Diese positive Beurteilung des zwischenstaatlichen Wettbewerbs wurde jedoch immer wieder in Frage gestellt. Break (1967), Boadway und Wildasin (1984), Wilson (1986), Zodrow und Mieszkowski (1986) und Keen und Marchand (1997) gelangen zu dem Ergebnis, dass Steuerwettbewerb einen zu starken Druck auf Steuersätze ausübt. Dadurch fallen die Steuersätze und die Steuereinnahmen im nicht-kooperativen Nash-Gleichgewicht zu niedrig aus. ${ }^{3}$ Die erzielten Steuereinnahmen reichen dann nicht mehr aus, um öffentliche Güter in optimaler Menge bereitzustellen, so dass es aufgrund des Steuerwettbewerbs zu einer Unterversorgung mit öffentlichen Gütern kommen wird. ${ }^{4}$ Oates und Schwab (1988, S. 334) halten diesem Argument jedoch entgegen, dass Bürger auch ein bestimmtes Niveau an öffentlichen Gütern wünschen, so dass ein wie oben skizzierter Steuerwettbewerb auch Kosten für die Bürger verursacht. Berücksichtigt man diese Kosten, so sei es nicht mehr sicher, ob das Ergebnis von Steuerwettbewerb notwendigerweise in einer Unterversorgung mit öffentlichen Gütern besteht. ${ }^{5}$ Sinn (1997, S. 27) und Wellisch (2000, S. 59) verweisen darauf, dass durch öffentliche Zwischenprodukte, wie Infrastruktur, Kapital angezogen wird, so dass bei diesen öffentlichen Gütern eher nicht mit einer Unterversorgung zu rechnen ist.

Eine weitere Quelle von Ineffizienzen wird in Nutzenspillovers, die von der Bereitstellung öffentlicher Güter ausgehen, gesehen. Musgrave (1971), Boadway

\footnotetext{
' Vgl. Brennan / Buchanan (1980, S. 171 f.), Oates / Schwab (1988, S. 333), Buchanan / Lee (1994, S. 221), Wrede (1998, S. 316; 1999, S. 177), Wellisch (2000, S. 17).

${ }^{2}$ Vgl. Nowotny (1999, S. 404), Flochel / Madies (2002, S. 122).

${ }^{3}$ Vgl. Wilson (1986, S. 296), Wildasin (1988, S. 229), Bucovetsky / Wilson (1991, S. 333), Keen / Marchand (1997, S. 33), Tausch (1998, S. 328).

${ }^{4}$ Vgl. Wilson (1986, S. 296 und S. 309), Zodrow / Mieszkowski (1986, S. 357 und S. 368 f.), Wildasin (1988, S. 229), Bucovetsky / Wilson (1991, S. 333), DePater / Myers (1994, S. 66), Fuest (1995, S. 478; 1997, S. 174), Sinn (1997, S. 15), Bucovetsky et al. (1998, S. 368), Hange / Wellisch (1998, S. 315), Brueckner (1999, S. 206).

${ }^{5}$ Vgl. Oates / Schwab (1988, S. 334).
} 
und Wildasin (1984), McLure (1986), Wellisch (1993 und 2000) und Bjorvatn und Schjelderup (2002) untersuchen das Phänomen, dass einige öffentliche Güter, die von einer Region bereitgestellt werden, auch einen Nutzen in anderen Regionen stiften. Da die Kosten und der Nutzen aus der Bereitstellung öffentlicher Güter dann auseinander fallen, ist das Prinzip der fiskalischen Äquivalenz durchbrochen.' Weil die Regionen den Nutzen des öffentlichen Gutes in den anderen Regionen nicht berücksichtigen, kommt es letztlich zu einer Unterversorgung mit öffentlichen Gütern. ${ }^{2}$ Daher werden eine überregionale Politikkoordinierung und/oder Ausgleichszahlungen gefordert, um diese externen Effekte zu internalisieren. $^{3}$

Weitere Ineffizienzen sind bei Vorliegen von ungleichen Regionen zu erwarten. Fragestellungen, die die Problematik der ungleichen Regionen aufgreifen, wurden beispielsweise von Flatters et al. (1974), Bucovetsky (1991), Wilson (1991), Kanbur und Keen (1993), DePater und Myers (1994), Arnold und Fuest (1999), Eggert und Haufler (1999) und Tausch (1998) untersucht. DePater und Myers (1994) zeigen, dass bei asymmetrischen, nicht mengenanpassenden Jurisdiktionen Ineffizienzen auftreten, da eine Jurisdiktion die Wohlfahrt einer anderen Jurisdiktion durch den Zinssatz beeinflusst, ohne diesen externen Effekt in ihren Entscheidungsprozess einzubeziehen. Bucovetsky (1991), Wilson (1991), Kanbur und Keen (1993) und Wellisch (2000) legen dar, dass die Position von kleinen Regionen in einem nicht-kooperativen Nash-Gleichgewicht besser ist als die von großen Regionen. Die kleine Region unterbietet den Steuersatz der großen Region und erzielt so eine höhere pro Kopf Wohlfahrt als bei kooperativem Verhalten. ${ }^{4}$ Es kommt zu einer Unterversorgung mit dem öffentlichen Konsumgut in der großen Region. ${ }^{5}$ Tausch (1998) zeigt allerdings, dass kleine Regionen nur bezüglich öffentlicher Konsumgüter von nicht- kooperativem Steuerwettbewerb profitieren. In einem Model mit öffentlichen Zwischenprodukten stellt sich

\footnotetext{
'Vgl. Musgrave (1971, S. 5), Nowotny (1999, S. 127).

${ }^{2}$ Vgl. Boadway / Wildasin (1984, S. 502), Wellisch (2000, S. 19), Bjorvatn / Schjelderup (2002, S. 111).

${ }^{3} \mathrm{Vgl}$. Boadway / Wildasin (1984, S. 519), McLure (1986, S. 343), Wellisch (1993, S. 677 f.), Richter / Seitz / Wiegard (1996, S. 15).

${ }^{4}$ Vgl. Bucovetsky (1991, S. 179 ff.), Wilson (1991, S. 445 f.), Kanbur / Keen (1993, S. 890), Tausch (1998, S. 329), Wellisch (2000, S. 73).

${ }^{5}$ Vgl. Bucovetsky (1991, S. 179).
} 
heraus, dass die kleinen Regionen aufgrund der Nichtrivalität des öffentlichen Zwischenprodukts immer benachteiligt sind. ${ }^{1}$

\subsection{Problemstellung der Arbeit}

In der vorliegenden Arbeit soll nun - in Abgrenzung zu der Vielzahl zuvor genannter Untersuchungen - der fiskalische Wettbewerb um Kapital zwischen eigennützigen Regierungen analysiert werden. In Abschnitt 1.2.1 wird daher erläutert, welche Kapitalmarktmodelle in der Theorie zum Steuer- und Standortwettbewerb Verwendung finden, und die Frage formuliert, welches dieser Modelle unter dem Kriterium der Darstellung von Wettbewerb um Kapital vorzuziehen ist.

Im Gegensatz zu anderen Arbeiten befasst sich diese Arbeit ausdrücklich mit fiskalischem Wettbewerb und nicht nur mit Steuerwettbewerb. Die Untersuchung bezieht sich insofern insbesondere auch auf den Ausgabenwettbewerb, den Regierungen untereinander eingehen. Deshalb wird in Abschnitt 1.2.2 die Rolle der öffentlichen Güter in der Literatur zum Steuer- und Standortwettbewerb thematisiert und die damit verbundene Problematik diskutiert.

In Abgrenzung zur Standardliteratur werden in dieser Arbeit eigennützige Regierungen modelliert. Um die Bedeutung und Begründung dieser Modellierung aufzuzeigen, werden in Abschnitt 1.2.3 die entscheidungstheoretischen Modellierungen von Regierungen in der Literatur dargestellt und diskutiert.

\subsubsection{Exogener Kapitalbestand oder vollkommen preiselastisches Kapitalange- bot}

In der Literatur zum Steuer- und fiskalischen Standortwettbewerb wird der Kapitalmarkt auf unterschiedliche Weise abgebildet. Grob lassen sich zwei Richtungen unterscheiden: Die erste Richtung nimmt einen exogen vorgegebenen Nettozinssatz an und unterstellt somit ein vollkommen preiselastisches Kapitalangebot. Die zweite Richtung geht davon aus, dass der Nettozinssatz endogen im Wettbewerb zwischen den Staaten bestimmt wird. Modelle dieser Richtung

' Vgl. Tausch (1998, S. 339 f.). 
zeichnen sich dadurch aus, dass sie stets einen exogen vorgegebenen Kapitalbestand annehmen. Daher werden sie im Weiteren als Modelle, die einen exogenen Kapitalbestand annehmen, bezeichnet, wohlwissend dass einige Modelle ${ }^{1}$ der ersten Richtung neben der Annahme eines exogen vorgegebenen Nettozinssatzes auch einen exogen vorgegebenen Kapitalbestand unterstellen.

Die meisten Aufsätze - wie beispielsweise die von Wilson (1986), Zodrow und Mieszkowski (1986), Braid (1996), Edwards und Keen (1996), Richter, Seitz und Wiegard (1996), Fuest (1997), Keen und Marchand (1997), Sinn (1997), Bucovetsky et al. (1998), Rauscher (1998) und Apolte (2001) - verwenden ein Kapitalmarktmodell, das von einem exogen vorgegebenen Nettozinssatz ausgeht. Begründet wird diese Annahme mit der Betrachtung eines oder vieler, kleiner Länder. ${ }^{2}$ Die Wirkung von Politikmaßnahmen eines kleinen Landes auf die übrigen Regionen ist so gering, dass sie von der Regierung dieses Landes bei ihren Entscheidungen vernachlässigt wird. ${ }^{3}$ Sinn (1997) betrachtet nur exemplarisch ein kleines Land und blendet die übrigen Regionen aus seiner Betrachtung aus. Wilson (1986), Zodrow und Mieszkowski (1986), Braid (1996), Edwards und Keen (1996), Richter, Seitz und Wiegard (1996), Fuest (1997), Keen und Marchand (1997), Rauscher (1998) und Apolte (2001) hingegen untersuchen eine Vielzahl von Regionen, die sich alle dadurch auszeichnen, dass jede Region zu klein ist, um auf die übrigen Konkurrenzregionen einen signifikanten Einfluss auszuüben.

Modelle, die von einem exogen vorgegebenen Kapitalbestand ausgehen und dabei die Wirkungen der Politikmaßnahmen einer Region auf die andere Region modellieren, werden demgegenüber beispielsweise von Wildasin (1989 und 1991), Bucovetsky (1991), DePater und Myers (1994), Hange und Wellisch (1998), Arnold und Fuest (1999), Roloff (1999) und Apolte (2001) verwendet. Wildasin (1989 und 1991) bezieht seine Betrachtungen auf die gesamte Ökonomie, so dass er deren Kapitalbestand als exogen gegeben ansieht. Andere Autoren hingegen beschränken ihre Betrachtungen auf eine Nation bzw. auf einen Staat, weshalb sie unterstellen, dass die Nation als Ganzes einen gegebenen Ka-

\footnotetext{
${ }^{1} \mathrm{Vgl}$. beispielsweise Zodrow / Mieszkowski (1986, S. 358).

${ }^{2}$ Vgl. Wilson (1986, S. 298), Edwards / Keen (1996, S. 119), Richter / Seitz / Wiegard (1996, S. 14 f.).

${ }^{3}$ Vgl. Oates / Schwab (1988, S. 337), Bucovetsky (1991, S. 167). 
pitalbestand hat. ${ }^{1}$ Wie auch immer die Bezugseinheit gewählt wird, ob als Nation, Menge von Jurisdiktionen oder gar als gesamte Ökonomie, allen Ansätzen ist gemeinsam, dass sie einen Wirtschaftsraum betrachten, dessen Kapitalbestand exogen vorgegeben ist.

Die beiden Kapitalmarktmodelle - zum einen mit der Annahme eines exogenen Kapitalbestandes und zum anderen mit der Annahme eines exogenen Nettozinssatzes - existieren in der Literatur zum Steuer- und Standortwettbewerb nebeneinander. Ein Grund für die Wahl des Kapitalmarktmodells ist in den Aufsätzen zum Steuer- und Standortwettbewerb eigentlich nicht zu finden. Daher wird im zweiten Kapitel dieser Arbeit untersucht, ob eines der Modelle vorzuziehen ist. $\mathrm{Da}$ es sich hier um Modelle zum Steuer- und Standortwettbewerb handelt, wird als Auswahlkriterium herangezogen, inwieweit ein Kapitalmarktmodell in der Lage ist, Steuer- bzw. Standortwettbewerb abzubilden. In Kapitel 2 wird also die Frage geklärt, durch welches der beiden Kapitalmarktmodelle Steuer- bzw. Standortwettbewerb konsistent dargestellt werden kann.

\subsubsection{Die Rolle der öffentlichen Güter}

In der Literatur zum Steuer- und Standortwettbewerb werden öffentliche Güter auf unterschiedliche Weise berücksichtigt. Die meisten Aufsätze - wie beispielsweise die von Flatters et al. (1974), Wiegard (1980), Bewley (1981), Brueckner (1983), Mintz und Tulkens (1986), Bucovetsky und Wilson (1991), Wildasin (1991), Krelove (1993), DePater und Myers (1994), Braid (1996), Yang (1996), Nechyba (1997), Hange und Wellisch (1998), Laussel und Le Breton (1998), Wrede (1998), Eggert (1999), Homburg (1999) und Fuest (2000) modellieren ein öffentliches Konsumgut, das von den einzelnen Regierungen ohne direktes Nutzenentgelt der Bevölkerung bereitgestellt wird. Dieses öffentliche Gut geht gemeinsam mit einem privaten Gut in die Nutzenfunktion der Haushalte ein. ${ }^{2}$ Der Staat erhöht also durch die zusätzliche Bereitstellung des

\footnotetext{
${ }^{1}$ Vgl. Bucovetsky (1991, S. 169), DePater / Myers (1994, S. 69), Hange / Wellisch (1998, S. 317).

${ }^{2}$ Vgl. Mintz / Tulkens (1986, S. 135), Wilson (1986, S. 299), Myers (1990, S. 109), Bucovetsky (1991, S. 169), Wildasin (1991, S. 397), Braid (1996, S. 1283), Yang (1996, S. 324), Nechyba (1997, S. 362), Hange / Wellisch (1998, S. 318), Wrede (1998, S. 317). 
öffentlichen Konsumgutes ausschließlich den Nutzen der Haushalte. ${ }^{1}$ Die meisten dieser Aufsätze unterstellen darüber hinaus, dass die Haushalte immobil sind. ${ }^{2}$ Insofern wirkt sich die Bereitstellung des öffentlichen Gutes nicht auf die Höhe der Einnahmen des Staates aus. Dadurch wird modelltheoretisch die Möglichkeit ausgeschlossen, dass Regierungen öffentliche Güter im Wettbewerb als strategische Variable einsetzen, um die Besteuerungsbasis zu verbreitern. Im Budget der jeweiligen Regierungen entstehen folglich nur zusätzliche Ausgaben durch die Bereitstellung einer zusätzlichen Einheit des öffentlichen Gutes.

Autoren - wie Richter (1994), Fuest (1995), Michael (1995), Richter, Seitz und Wiegard (1996), Richter und Wellisch (1996), Keen und Marchand (1997), Sinn (1997), Arnold und Fuest (1999), Tausch (1999), Roloff (1999) und Apolte (2001) - modellieren demgegenüber ein öffentliches Gut, welches kein Konsumgut, sondern ein Zwischenprodukt ist. Das öffentliche Zwischenprodukt geht dann in die Produktion ein und erhöht die Produktivität der übrigen privaten Primärfaktoren. ${ }^{3}$ Die Menge an öffentlichem Input, die von der jeweiligen Regierung ohne direktes Nutzenentgelt den Unternehmen zur Verfügung gestellt wird, beeinflusst somit die Menge an Kapital, die in einer Region eingesetzt wird. ${ }^{4}$ Die Regierungen können demnach durch die Bereitstellung von öffentlichen Inputs die Höhe der Besteuerungsbasis beeinflussen. Darüber hinaus können sie das öffentliche Zwischenprodukt dazu verwenden, um die Attraktivität ihres Standortes zu verbessern. ${ }^{5}$ Die Attraktivität hängt also nicht nur von der Höhe der Steuerbelastung einer Region, sondern auch von deren Ausstattung mit öffentlichen Zwischenprodukten ab. Dieser Aspekt des fiskalischen Standortwettbewerbs kann jedoch nur analysiert werden, wenn ein öffentliches Zwischenprodukt und dessen Wirkung insbesondere auf Kapital modelliert wird. Daher wird in Kapitel 3 das Modell von Kapitel 2 durch die Berücksichtigung eines öffentlichen Zwischenprodukts erweitert. Im Gegensatz zu anderen Modellen, wie beispielsweise das von Fuest (1995), Hange und Wellisch (1998), Arnold und Fuest (1999) und Homburg (1999), wird hier zunächst noch auf eine

\footnotetext{
${ }^{1}$ Vgl. beispielsweise Mintz / Tulkens (1986, S. 135), Braid (1996, S. 1283).

${ }^{2}$ Vgl. beispielsweise Bucovetsky (1991, S. 168), Bucovetsky / Wilson (1991, S. 335), DePater / Myers (1994, S. 69), Braid (1996, S. 1281), Hange / Wellisch (1998, S. 316), Eggert (1999, S. 3), Homburg (1999, S. 3), Fuest (2000, S. 359).

${ }^{3}$ Vgl. Fuest (1995, S. 480 f.), Michael (1995, S. 74), Roloff (1997; 1999, S. 50), Tausch (1998, S. 329 und S. 331).

${ }^{4}$ Vgl. Roloff (1997; 1999, S. 50), Tausch (1998, S. 337 f.), Arnold / Fuest (1999, S. 170).

${ }^{5}$ Vgl. Richter / Seitz / Wiegard (1996, S. 13). 
Modellierung der Regierung verzichtet. Es soll an dieser Stelle allein die Wirkung der fiskalpolitischen Instrumente - Kapitalsteuer und öffentliches Zwischenprodukt - auf dem Kapitalmarkt betrachtet werden. Durch die Fokussierung auf den Untersuchungsgegenstand Kapitalmarkt kann nämlich die Substituierbarkeit der beiden fiskalpolitischen Instrumente und die sich daraus ergebenden Konsequenzen bezüglich Kapitallokation und Nettozinssatz herausgearbeitet werden.

Wird in der Literatur zum Steuer- und Standortwettbewerb ein öffentliches Zwischenprodukt modelliert, so wird in der Regel angenommen, dass dieses Gut kein rein öffentliches Gut im Sinne von Samuelson ist. ' Staatliche Infrastruktur wird als unreines öffentliches Gut mit einer mehr oder weniger stark ausgeprägten Nutzungsrivalität aufgefasst. ${ }^{2}$ Bei der Nutzung dieses Gutes entstehen dann Stauungskosten in Form einer Behinderung der übrigen Nutzer. ${ }^{3}$ Colombier (2001) hat jedoch nachgewiesen, dass allein faktorvermehrende öffentliche Zwischenprodukte mit den Axiomen der Produktionstheorie kompatibel sind. Er zeigt, dass die Gutseigenschaften nicht mit der angenommenen Aggregationsebene variieren dürfen und es sich insofern nur beim „unpaid factor" und beim „factor-augmenting public input" um konsistente produktionstheoretische Modellierungen handelt. ${ }^{4} \mathrm{Da}$ der „unpaid factor“ letztlich ein privates, d.h. rivalisierendes Gut ist, welches staatlich bereitgestellt wird, kann zur Modellierung eines öffentlichen Zwischenproduktes letztlich nur der ,factor-augmenting public input" verwendet werden. ${ }^{5}$ Denn im Gegensatz zu anderen Modellierungen von öffentlichen Inputs zeichnet sich der „factor-augmenting public input" dadurch aus, dass er sowohl auf der Branchen-, als auch auf der Unternehmens- als auch auf der Faktorebene nichtrivalisierend ist. ${ }^{6}$ Die Annahme von Stauungskosten ist damit also nicht vereinbar. ${ }^{7}$ Aus der Nicht-Rivalitätseigenschaft des „factor-

\footnotetext{
' Vgl. beispielsweise Burbidge / Myers (1994, S. 444), Fuest (1995, S. 481), Keen / Marchand (1997, S. 36), Sinn (1997, S. 16 f.).

${ }^{2}$ Vgl. z.B. Sinn (1997, S. 16 f.).

${ }^{3}$ Vgl. Richter (1994, S. 326), Richter / Seitz / Wiegard (1996, S. 16), Richter / Wellisch (1996, S. 76), Keen / Marchand (1997, S. 36), Sinn (1997, S. 16 f.), Tausch (1998, S. 329 und S. 331), Apolte (2001, S. 361).

${ }^{4}$ Vgl. Colombier / Pickhardt (1998, S. 22), Colombier (2001, S. 78).

${ }^{5}$ Vgl. Colombier / Pickhardt (1998, S. 22), Colombier (2001, S. 78).

${ }^{6} \mathrm{Vgl}$. Colombier (2001, S. 44).

${ }^{7} \mathrm{Vgl}$. Colombier (2001, S. 79). 
augmenting public input" resultieren steigende Skalenerträge. ${ }^{1}$ Aufgrund des Nachweises von Colombier (2001) wird in dieser Arbeit im Gegensatz zu bisherigen Aufsätzen zu dem Thema Steuer- und Standortwettbewerb ein „factoraugmenting public input" modelliert. Die vorliegende Arbeit unterscheidet sich somit von anderen Aufsätzen dadurch, dass explizit steigende Skalenerträge verwendet werden und von der Annahme von Stauungskosten Abstand genommen wird.

\subsubsection{Formen eigennütziger Regierungen}

Abgesehen von Aufsätzen mit einem wohlwollenden und allwissenden Planer ${ }^{2}$ bezieht sich der größte Teil der Literatur zum fiskalischen Wettbewerb auf wohlwollende Regierungen. ${ }^{3}$ Dabei wird angenommen, dass die einzelnen, sich im Wettbewerb befindenden Regierungen über die Interessen ihrer Bürger informiert sind und diese in ihrer Politik umsetzen. ${ }^{4}$ Weshalb Regierungen das Ziel verfolgen sollten, den Präferenzen ihrer Bürger bestmöglich gerecht zu werden, wird in der Regel mit der Bedeutung der Wiederwahl für die Regierungen begründet. Diesbezüglich ist das Medianwählermodell heutzutage die Standardannahme. ${ }^{5}$ Oates und Schwab (1988) verwenden beispielsweise das Medianwählermodell $^{6}$ als Mechanismus, um die Fiskalpolitik von Regierungen zu bestimmen. Da sie davon ausgehen, dass die Bürger einer Jurisdiktion identisch sind, maximieren die Regierungen letztlich den Nutzen eines repräsentativen

\footnotetext{
${ }^{1}$ Vgl. Colombier / Pickhardt (1998, S. 16), Colombier (2001, S. 78), Roloff (2001, S. 116 f.), Feehan / Matsumoto (2002, S. 61).

${ }^{2}$ Vgl. beispielsweise Richter / Seitz / Wiegard (1996, S. 21).

${ }^{3}$ Vgl. Sinn (1997, S. 11), Rauscher (1998, S. 59), Eggert (1999, S. 1). Die Annahme einer wohlwollenden Regierung wird beispielsweise getätigt von Oates (1972), Flatters et al. (1974, S. 101), Wiegard (1980, S. 188), Gordon (1983, S. 567 ff.), Wilson (1986, S. 300), Zodrow / Mieszkowski (1986, S. 359), Oates / Schwab (1988), Myers (1990, S. 111), Bucovetsky (1991, S. 170), Bucovetsky / Wilson (1991, S. 338), Hercowitz / Pines (1991, S. 170), Wildasin (1991), DePater / Myers (1994), Fuest (1995), Braid (1996, S. 1283), Richter / Wellisch (1996, S. 74), Yang (1996, S. 324), Keen / Marchand (1997), Tausch (1998, S. 329), Arnold / Fuest (1999), Eggert / Haufler (1999, S. 87), Homburg (1999).

${ }^{4}$ Vgl. Wilson (1986, S. 300; 1991, S. 428), Zodrow / Mieszkowski (1986, S. 359), Bucovetsky / Wilson (1991, S. 338), Eggert (1999, S. 1).

${ }^{5} \mathrm{Vgl}$. Niskanen (1997, S. 466).

${ }^{6}$ Siehe für die Erläuterung dieses Modells beispielsweise Atkinson / Stiglitz (1980, S. 299 ff.).
} 
Bürgers. ' Solchen Modellierungen liegt eigentlich die Vorstellung von einer eigennützigen Regierung zugrunde. Da diese Regierungen jedoch durch Wahlen vollkommen restringiert sind, erreichen sie ihr Nutzenmaximum, indem sie exakt den Wünschen der Wählermehrheit entsprechen, d.h. indem sie sich wie wohlwollende Regierungen verhalten. ${ }^{2}$ Die Regierungen erscheinen letztlich als wohlwollend, obwohl sie eigentlich eigennützige Regierungen unter vollkommener Wiederwahlrestriktion sind. ${ }^{3}$

Seit der Veröffentlichung des Buches "The Power to Tax“ von Brennan und Buchanan (1980) haben vereinzelt Autoren ${ }^{4}$ die Idee, Regierungen als Leviathanregierungen zu modellieren, aufgegriffen und diese in ihre Modelle zum fiskalischen Standortwettbewerb integriert. Das Ziel der Regierungen besteht dann nicht darin, die gesellschaftliche Wohlfahrt zu maximieren, sondern die Rente aus der Regierungstätigkeit. ${ }^{5}$ Das Leviathan-Modell basiert insofern auf der Annahme, dass die Wiederwahlchancen keine Restriktion für die Regierungen darstellen. ${ }^{6}$ Dieses Regierungsmodell ist somit der exakte Gegensatz zu der zuvor diskutierten Annahme einer wohlwollenden und allwissenden Regierung entsprechend des Medianwähleransatzes. In gleicher Weise allerdings wie der Medianwähleransatz die Ohnmacht der Politiker gegenüber ihren Bürgern stark überzeichnet, so überschätzt das Modell des Leviathans die Macht der Regierenden.

Im Gegensatz zur Modellierung einer wohlwollenden Regierung eröffnet die Modellierung der Regierung als Leviathan jedoch die Möglichkeit, die unmittelbare Anreizwirkung des Wettbewerbs auf eigennützige Regierungen zu betrachten. Bei der Modellierung der Regierung als Leviathan liegt die Beschränkung alleinig im Standortwettbewerb. Dagegen werden wohlwollende, Wählerstimmen maximierende Regierungen auch durch die Wählerwünsche in ihren Handlungen beschränkt, deren Erfüllung dann vom Wettbewerb der Regionen beeinflusst wird. Um die unmittelbare Anreizwirkung von zwischenstaatlichem Wettbewerb auf eigennützige Regierungen zu analysieren, werden in dieser Untersuchung Regierungen als Leviathanregierungen stilisiert, wohlwissend dass es sich

\footnotetext{
${ }^{1}$ Vgl. Oates / Schwab (1988, S. 339).

${ }^{2}$ Vgl. Brennan (1997, S. 95), Blankart (2001, S. 31).

${ }^{3} \mathrm{Vgl}$. Roloff (2001, S. 57).

${ }^{4}$ Vgl. beispielsweise Kamada (1989), Rauscher (1998), Eggert (1999, S. 3), Wrede (1999), Apolte (2001, S. 359), Flochel / Madies (2002).

${ }^{5}$ Vgl. Beckmann / Lackner (1998, S. 2).

${ }^{6}$ Vgl. Beckmann / Lackner (1998, S. 2).
} 
dabei lediglich um eine erste Annäherung an ein adäquates entscheidungstheoretisches Modell handeln kann.

Weit verbreitet ist bei Autoren, die Leviathanregierungen im Wettbewerb modellieren, die Ansicht, dass der Wettbewerb zwischen den Institutionen geeignet ist, um die Steuermonopolstellung der Leviathanregierungen zu reduzieren oder gar zu durchbrechen.' So argumentieren Brennan und Buchanan (1980), dass der Wettbewerb zwischen den Regierungen im Kontext von zwischenstaatlicher Mobilität der Produktionsfaktoren als Substitut zu einer Finanzverfassung zur Beschränkung des Steuermonopols fungieren kann. ${ }^{2}$ Apolte (2001) ging der Frage nach, ob diese von Brennan und Buchanan aufgestellte These auch bei Vorliegen von Immobilität eines Produktionsfaktors haltbar ist. Er kam zu dem Ergebnis, dass eine marktmäßige Zähmung des Leviathans durch den Wettbewerb der Institutionen dann nicht zu erwarten ist. ${ }^{3}$ Rauscher (1998) stellt fest, dass bei Vorliegen von ungleichen Regionen ebenfalls nicht mit der von Brennan und Buchanan propagierten Zähmung des Leviathans durch den Wettbewerb der Institutionen zu rechnen ist. Kamada (1989) untersuchte Anreize für eine Steuerharmonisierung zwischen Ländern, Gebietskörperschaften und lokalen Leviathanregierungen. Wrede (1999) wählt ein dynamisches Spiel zwischen Leviathanregierungen, um horizontale und vertikale Externalitäten im Steuerwettbewerb zu analysieren. Flochel und Madies (2002) stellen ein Modell auf, durch welches horizontaler Steuerwettbewerb und vertikale Interaktionen zwischen Leviathanregierungen mit gemeinsamer Besteuerungsbasis betrachtet werden können. Die zuvor genannten Autoren beschränken ihre Betrachtungen in erster Linie auf den Steuerwettbewerb. Die Möglichkeit, durch zusätzliche Ausgaben in öffentliche Zwischenprodukte Kapital zu attrahieren und darüber die Steuereinnahmen zu erhöhen, wird vernachlässigt. Dieser Thematik soll deshalb im 4. Kapitel der Arbeit insbesondere nachgegangen werden. Dort soll ausschließlich der Ausgabenwettbewerb isoliert modelliert werden, weshalb angenommen wird, dass die Steuern in den Regionen bereits harmonisiert sind. Dadurch kann dann herausgearbeitet werden, wie Leviathanregierungen die Bereitstellungsmenge an öffentlichem Zwischenprodukt strategisch einsetzen, um ihren Surplus resp. Eigennutz zu maximieren.

\footnotetext{
${ }^{1}$ Vgl. Brennan / Buchanan (1980, S. 171 f.), Oates / Schwab (1988, S. 333), Buchanan / Lee (1994, S. 221), Fuest (1997, S. 175), Eggert (1999, S. 3), Wrede (1999, S. 177).

${ }^{2}$ Vgl. auch Oates / Schwab (1988, S. 333 f.), Sinn (1992, S. 187), Hoyt (1999, S. 155 f.).

${ }^{3}$ Vgl. Apolte (2001, S. 377). 
Die Modellierung einer eigennützigen Regierung im Sinne einer Regierung, die weder eine Leviathanregierung noch eine wohlwollende Regierung ist, wurde von Edwards und Keen (1996) in die Literatur zum Steuer- und Standortwettbewerb eingeführt. Sie verwenden Regierungen, die sowohl die Wohlfahrt ihrer Bürger in Betracht ziehen als auch den Surplus berücksichtigen, den sie erwirtschaften können. ${ }^{1}$ In dieser Arbeit wird dieser Modellierung des Staates jedoch nicht gefolgt und der Staat als Leviathan dargestellt. Die Modellierung des Staates als Leviathan wird gewählt, weil dadurch die Fokussierung auf die durch den Standortwettbewerb ausgelösten Effekte möglich ist. Würde etwa eine eigennützige repräsentative Regierung, wie sie Edwards und Keen aufzeigen, angenommen, so würde die Regierung nicht nur durch den fiskalischen Wettbewerb in ihrem Streben nach maximalem Nutzen beschränkt, sondern darüber hinaus auch noch durch die Präferenzen der Wähler. Somit könnte die Situation entstehen, dass ein Anreiz, der auf den Standortwettbewerb zurückzuführen ist, letztlich in der Analyse nicht in Erscheinung tritt, weil er durch einen gegenläufigen, von den Wählern verursachten Anreiz kompensiert wird. In dieser Arbeit sollen allein Anreize des Standortwettbewerbs Berücksichtigung finden. Daher wird der Untersuchungsgegenstand entsprechend eingegrenzt und als entscheidungstheoretische Referenzfigur eine Leviathanregierung gewählt, die einzig und allein durch den Wettbewerb der Regionen um mobile Faktoren und durch exogen vorgegebene Finanzverfassungsregeln, die diesen Wettbewerb betreffen, in ihren Handlungen eingeschränkt wird. Mit der Verwendung dieser Annahme soll also nicht die Bedeutung von Wahlen für die Entscheidungen der Regierungen verneint werden, sondern lediglich zur Präzisierung der Analyse von der Betrachtung weiterer beschränkender Faktoren außer dem Standortwettbewerb abgesehen werden.

\subsection{Gliederung der Arbeit}

Die Arbeit ist wie folgt gegliedert: In Kapitel 2 wird der Frage nachgegangen, durch welches Kapitalmarktmodell ein Steuerwettbewerb um Kapital adäquat abgebildet werden kann. Hierzu werden die beiden in der Literatur am häufigs-

\footnotetext{
${ }^{\prime}$ Vgl. Edwards / Keen (1996, S. 115), Hange / Wellisch (1998, S. 315), Wrede (1998, S. 316), Fuest (2000, S. 358), Roloff (2001, S. 56).
} 
ten verwendeten Modelle analysiert, und zwar das Modell eines kleinen Landes, bei dem das Kapitalangebot vollkommen preiselastisch ist, und das Modell, bei dem von einem exogen vorgegebenen Kapitalbestand ausgegangen wird. Dieses Kapitel liefert somit eine Begründung für das in der folgenden Untersuchung herangezogene Kapitalmarktmodell.

In Kapitel 3 wird dann die Wirkung von Kapitalertragsteuern und öffentlichen Zwischenprodukten auf dem Kapitalmarkt einer Föderation untersucht. Auf Basis einer Analyse über die getrennte Wirkung von Steuern und öffentlichen Inputs wird das Ausmaß der Substituierbarkeit der beiden fiskalpolitischen Instrumente bestimmt. Darauf aufbauend wird ein Wert ermittelt, der das Niveau beider fiskalpolitischer Mittel zusammengefasst beschreibt und der als fiskalpolitischer Instrumenten-Index bezeichnet wird. Mit seiner Hilfe wird dann der Frage nachgegangen, unter welchen Bedingungen eine Steuersatzsenkung einer Region zu einer höheren Kapitalmenge in eben dieser Region führt.

Die Fragestellung von Kapitel 4 ist von der Erkenntnis des Kapitels 3 bezüglich der Wichtigkeit des Ausgabenwettbewerbs motiviert. Kapitel 4 setzt sich mit der Frage auseinander, welche Wirkung eine Steuerharmonisierung auf Leviathanregierungen im Ausgabenwettbewerb entfaltet. Im Rahmen eines ZweiRegionen-Modells werden die Regierungen als Leviathanregierungen im nichtkooperativen Nash-Spiel dargestellt. Es wird untersucht, inwiefern die Menge an öffentlichem Zwischenprodukt, die diese Regierungen freiwillig bereitstellen, bereits durch den von der Verfassung vorgegebenen, harmonisierten Steuersatz indirekt festgelegt wird.

Kapitel 5 enthält das Fazit der Arbeit sowie offene Fragen und Probleme bezüglich des Themas.

\section{Modellierung des Kapitalmarktes im Steuerwettbewerb}

In der Literatur zum Steuer- und Standortwettbewerb kommen unterschiedliche Kapitalmarktmodelle zur Anwendung.' So finden sich Modelle, die einen exogen vorgegebenen Kapitalbestand annehmen und/oder die von einem konstanten

${ }^{1}$ Vgl. Hensberg (2002). 
Nettozinssatz ausgehen. ${ }^{1}$ In diesem Kapitel soll der Frage nachgegangen werden, welche dieser Modelle einen Steuerwettbewerb um Kapital adäquat abbilden können. Dazu werden die beiden am häufigsten verwendeten Modelle gegenübergestellt und herausgearbeitet, inwiefern durch sie eine Konkurrenzsituation zwischen den Regionen beschrieben wird.

Kapitel 2 ist wie folgt strukturiert: In Abschnitt 2.1 wird zunächst ein Kapitalmarktmodell diskutiert, bei dem ein vollkommen preiselastisches Kapitalangebot unterstellt wird. Dazu wird in Abschnitt 2.1.1 mit Hilfe der Nachfrage- und Angebotsfunktion die Gleichgewichtsbedingung des Kapitalmarktes hergeleitet. Auf dieser Basis kann dann in Abschnitt 2.1.2 die Wirkung einer Steuersatzänderung einer Region i auf den Nettozinssatz und die Kapitallokation ermittelt und der Frage nachgegangen werden, inwieweit dieses Modell einen Steuerwettbewerb um Kapital abbilden kann. In Abschnitt 2.2 wird ein Kapitalmarktmodell untersucht, bei dem der Kapitalstock als exogen vorgegeben und fix angenommen wird. Aus der gesamtwirtschaftlichen Kapitalangebots- und Nachfragefunktion wird dafür in Abschnitt 2.2.1 die Bedingung für ein Gleichgewicht auf dem Kapitalmarkt bestimmt. Darauf aufbauend kann dann in Abschnitt 2.2.2 geprüft werden, inwieweit eine Steuersatzänderung in einer der Regionen zu einer Kapitalrelokation und/oder zu einer Nettozinssatzanpassung führt. Dadurch kann festgestellt werden, inwiefern dieses Modell einen Steuerwettbewerb um Kapital darzustellen vermag. Das Kapitel 2 endet mit einer Zusammenfassung in Abschnitt 2.3.

\subsection{Vollkommen preiselastisches Kapitalangebot}

In Modellen, die ein vollkommen preiselastisches Kapitalangebot annehmen, wird in der Regel folgende Situation modelliert: Die Welt besteht aus einer endlichen Anzahl (n) von Regionen. ${ }^{2}$ Betrachtet wird beispielhaft eine der kleinen Regionen (i). ${ }^{3}$ In dieser Region kommen die Produktionsfaktoren Arbeit (L) und Kapital (K) zum Einsatz. Der Faktor Arbeit ist vollständig immobil. Kapital

\footnotetext{
I Vgl. beispielsweise Zodrow / Mieszkowski (1986), Wildasin (1991), Richter / Wellisch (1993), Edwards / Keen (1996), Fuest (1997), Sinn (1997), Hange / Wellisch (1998), Tausch (1998), Apolte (2001).

${ }^{2}$ Vgl. Oates / Schwab (1988, S. 336).

${ }^{3}$ Vgl. Sinn (1997, S. 14).
} 
wird als vollkommen mobil unterstellt. ${ }^{1}$ In jeder Region ist eine exogen vorgegebene Zahl von identischen Unternehmen angesiedelt, die das Einheitsgut (Y) herstellen. Dieses Einheitsgut dient als numéraire mit einem Preis von Eins. ${ }^{2}$ Die Unternehmen benötigen für die Produktion ausschließlich die beiden Primärfaktoren, Arbeit und Kapital. Demnach entspricht die Produktionsfunktion einer Region $\mathrm{i}^{3}$

$$
Y_{i}=f_{i}\left(K_{i}, L_{i}\right) .
$$

Die Grenzerträge des Kapitals sind positiv und abnehmend: ${ }^{4}$

$$
\begin{aligned}
& \frac{\partial f_{i}\left(K_{i}, L_{i}\right)}{\partial K_{i}} \equiv f_{K i}\left(K_{i}\right)>0, \\
& \frac{\partial^{2} f_{i}\left(K_{i}, L_{i}\right)}{\partial K_{i}^{2}} \equiv f_{K i K i}\left(K_{i}\right)<0 .
\end{aligned}
$$

Die Regierungen der Regionen erheben Steuern auf das Kapital. Die Doppelbesteuerung wird nach dem Quellenlandprinzip vermieden. ${ }^{5}$ In diesem Kapitel wird die Analyse sowohl für eine Substanzsteuer als auch für eine Ertragsteuer durchgeführt. Im Fall einer Substanzsteuer erzielt die Region i Steuereinnahmen $\left(T_{i, s}\right)$, die sich aus dem Produkt des Steuersatzes $\left(t_{i, s}\right)$ und der in der Region eingesetzten Kapitalmenge $\left(\mathrm{K}_{\mathrm{i}}\right)$ ergeben:

$$
T_{i, s}=t_{i, s} \cdot K_{i} \text {. }
$$

Für den Fall einer Ertragsteuer belaufen sich die Steuereinnahmen $\left(\mathrm{T}_{\mathrm{i}, \mathrm{e}}\right)$ auf das Produkt aus Steuersatz $\left(\mathrm{t}_{\mathrm{i}, e}\right)$, Bruttozinssatz $\left(\mathrm{z}_{\mathrm{i}}\right)$ und eingesetzter Kapitalmenge $\left(\mathrm{K}_{\mathrm{i}}\right)$ :

$$
T_{i, e}=t_{i, e} \cdot z_{i} \cdot K_{i} \cdot
$$

Der Einfachheit halber wird angenommen, dass die Regionen darüber hinaus weder Einnahmen noch Ausgaben haben, die Einfluss auf den Kapitalmarkt nehmen.

\footnotetext{
${ }^{1}$ Vgl. Oates / Schwab (1988, S. 337), Sinn (1997, S. 14 und 18), Rauscher (1998, S. 61), Tausch (1998, S. 330). Für den Realitätsbezug dieser Annahme vergleiche auch Apolte (1999, S. $24 \mathrm{ff}$.$) .$

${ }^{2}$ Vgl. Tausch (1998, S. 330), Hensberg (2002, S. 5).

${ }^{3}$ Vgl. Sinn (1997, S. 14 und 18), Apolte (2001, S. 360).

${ }^{4}$ Vgl. Zodrow / Mieszkowski (1986, S. 358), Tausch (1998, S. 331), Hensberg (2002, S. 5).

${ }^{5}$ Vgl. Roloff et al. (1994, S. 61 ff.). 


\subsubsection{Kapitalmarktgleichgewicht}

Die Unternehmen treten als Nachfrager auf dem Kapitalmarkt auf. Sie verfolgen das Ziel, ihren Gewinn $\left(\Pi_{i}\right)$ zu maximieren. Dieser entspricht der Differenz aus ihren Einnahmen, die aufgrund eines Preises von Eins mit der Outputmenge übereinstimmen, und ihren Ausgaben, die bei der Entlohnung der Primärfaktoren anfallen. Als Gewinnfunktion erhält man also: ${ }^{1}$

$$
\Pi_{i}=f_{i}\left(K_{i}, L_{i}\right)-z_{i} \cdot K_{i}-w_{i} \cdot L_{i},
$$

wobei $w_{i}$ den Bruttolohnsatz und $z_{i}$ den Bruttozinssatz der Region i symbolisiert. Ein Gewinnmaximum liegt u.a. vor, wenn die Grenzproduktivität des Kapitals dem Bruttozinssatz entspricht: ${ }^{2}$

$$
z_{i}=f_{K i}\left(K_{i}\right) .
$$

Je nach dem, ob eine Kapitalertragsteuer oder eine Kapitalsubstanzsteuer zur Anwendung gelangt, besteht ein unterschiedlicher Zusammenhang zwischen Brutto- und Nettozinssatz. Im Fall einer Substanzsteuer auf das Kapital entspricht der Bruttozinssatz der Summe aus dem Nettozinssatz (r) und dem Steuersatz der Region i:

$$
z_{i}=r_{i}+t_{i, s} .
$$

Im Fall einer Ertragsteuer auf das Kapital erhält man den Bruttozinssatz wie folgt:

$$
z_{i}=\frac{r_{i}}{\left(1-t_{i, e}\right)} .
$$

Die Unternehmen maximieren also ihren Gewinn im Fall einer Kapitalsubstanzsteuer, wenn

$$
r_{i}+t_{i, s}=f_{K i}\left(K_{i}\right)
$$

gilt und im Fall einer Kapitalertragsteuer, wenn

$$
\frac{r_{i}}{\left(1-t_{i, e}\right)}=f_{K i}\left(K_{i}\right)
$$

vorliegt. Definiert man $f_{z l}$ als Umkehrfunktion von $f_{k l}$, dann kann die Menge an nachgefragtem Kapital in der Region i explizit bestimmt werden. Kommt eine Substanzsteuer zur Anwendung, so wird Kapital in Höhe von

\footnotetext{
${ }^{1}$ Vgl. Homburg (1999, S. 3), Kollintzas / Philippopoulos / Vassilatos (2000, S. 6), Apolte (2001, S. 361).

${ }^{2} \mathrm{Vgl}$. Apolte (2001, S. 361), Hensberg (2002, S. 6).
} 
(2.12)

$$
K_{i}=f_{z i}\left(r_{i}+t_{i, s}\right)
$$

und bei einer Ertragsteuer in Höhe von

$$
K_{i}=f_{z i}\left(\frac{r_{i}}{\left(1-t_{i, e}\right)}\right)
$$

nachgefragt. Weil Kapital eine nicht-negative Größe ist, gilt:

$$
f_{z i}\left(z_{i}\right) \geq 0 \text {. }
$$

Die Ableitung einer Umkehrfunktion entspricht genau dem Reziproken der Ableitung der Originalfunktion: ${ }^{1}$

$$
f_{z i z i}=\frac{1}{f_{K i K i}} \text {. }
$$

Insofern kann aus Gleichung (2.3) gefolgert werden, dass

$$
f_{\text {zizi }}\left(z_{i}\right)<0
$$

vorliegt. Somit entsteht c.p. eine zunehmende Nachfrage nach Kapital bei sinkendem Nettozinssatz und eine abnehmende Kapitalnachfrage bei steigendem Nettozinssatz. Der Zusammenhang zwischen dem Nettozinssatz und der Kapitalnachfrage ist also negativ.

Es wird angenommen, dass es sich bei den betrachteten Regionen um äußerst kleine Regionen handelt. Diese Regionen sind so klein, dass ihre Aktionen letztlich nahezu keine Auswirkungen auf den Nettozinssatz haben. In den Modellen, die solche kleine Regionen betrachten ${ }^{2}$, wird daher der Einfachheit halber unterstellt, dass diese Auswirkungen so gering sind, dass sie vernachlässigt werden können. Dann stellt der Nettozinssatz einen Parameter dar, der für eine kleine Region i exogen vorgegeben ist. ${ }^{3}$ Folglich wird in der Region $i$ in beliebiger Menge Kapital angeboten, wenn der Nettozinssatz dem exogen vorgegebenem Weltmarktzinssatz $(\bar{r})$ entspricht: ${ }^{4}$

$$
\text { (2.17) } r_{i}=\bar{r} \text {. }
$$

Insofern wird von einem vollkommen preiselastischen Kapitalangebot ausgegangen.

\footnotetext{
${ }^{1}$ Vgl. Chiang (1984, S. 173).

${ }^{2}$ Vgl. beispielsweise Oates / Schwab (1988, S. 337) und Sinn (1997, S. 14 und 18).

${ }^{3}$ Vgl. Oates / Schwab (1988, S. 337), Hensberg (2002, S. 13).

${ }^{4}$ Vgl. Sinn (1997, S. 14 und 18).
} 
Ein Gleichgewicht auf dem Kapitalmarkt der Region i ist nun genau dann gegeben, wenn folgende Bedingung für die gleichgewichtige Kapitalmenge $\left(K_{i}^{*}\right)$ erfüllt ist: ${ }^{1}$

$$
K_{i}^{*}=f_{z i}\left(\bar{r}+t_{i, s}\right)
$$

bzw.

$$
K_{i}^{*}=f_{z i}\left(\frac{\bar{r}}{\left(1-t_{i, e}\right)}\right)
$$

(vgl. Gleichung (2.17) und Gleichung (2.12) bzw. Gleichung (2.13)) .

\subsubsection{Wirkung einer Steuersatzänderung}

Eine besondere Eigenschaft dieses Kapitalmarktes ist es, dass der Nettozinssatz als exogen vorgegeben und fix angesehen werden kann. Dadurch hat eine marginale Änderung des Steuersatzes keinen Einfluss auf die Höhe des Nettozinssatzes:

$$
\frac{\partial \bar{r}}{\partial t_{i, s}}=0
$$

bzw.

$$
\frac{\partial \bar{r}}{\partial t_{i, e}}=0 .
$$

Die Anpassung des Kapitalmarktes an eine Steuersatzänderung erfolgt also ausschließlich durch Mengenanpassungsprozesse. Wird eine Substanzsteuer auf das Kapital erhoben, dann wird durch eine marginale Änderung des Steuersatzes der Region i Kapital in Höhe von

$$
\frac{\partial K_{i}}{\partial t_{i, s}}=f_{z i z i}\left(z_{i}\right)<0
$$

zu- bzw. abfließen (siehe Gleichung (2.18) und Gleichung (2.16)). Wird eine Ertragsteuer angewendet, so wird die Höhe der Kapitalmenge durch eine marginale Steuersatzänderung wie folgt verändert:

${ }^{1}$ Vgl. Sinn (1997, S. 14), Hensberg (2002, S. 13). 


$$
\frac{\partial K_{i}}{\partial t_{i, e}}=f_{z i z i}\left(z_{i}\right) \cdot \frac{\bar{r}}{\left(1-t_{i, e}\right)^{2}}<0
$$

(siehe Gleichung (2.19) und Gleichung (2.16)). Erhöht die Regierung der Region i also den Steuersatz auf das Kapital bzw. auf den Kapitalertrag, so sinkt die Kapitalmenge, die in Region i eingesetzt wird, und umgekehrt.

$\mathrm{Da}$ der Nettozinssatz von den steuerpolitischen Maßnahmen der Region i nicht beeinflusst wird, kommt es in den Konkurrenzregionen (j) zu keinerlei Veränderung der Kapitalmenge:

$$
\frac{\partial K_{j}}{\partial t_{i, s}}=0
$$

bzw.

$$
\frac{\partial K_{j}}{\partial t_{i, e}}=0 .
$$

Senkt die Region i ihren Steuersatz, so bleibt die Kapitalmenge bei einem so modellierten Kapitalmarkt in den Konkurrenzregionen unverändert. Ein Wettbewerb um Kapital greift nicht, weil auf einem solchen Kapitalmarkt der Konkurrenzregion kein Kapital entzogen werden muss, um selber zusätzliches Kapital einsetzen zu können. Die Wirkung der Steuersatzänderung der Region i ist demnach allein auf die Region selbst beschränkt, da alle Konkurrenzregionen von dieser Änderung unbeeinflusst bleiben. Verändert Region i ihren Steuersatz, so bleiben die Konkurrenzregionen davon unberührt. Insofern kann nicht von einer Konkurrenzsituation zwischen den Regionen gesprochen werden. Diese Eigenschaft des Kapitalmarktes führt dazu, dass die Reaktionen auf diesem Kapitalmarkt grafisch für die Region i einzeln verdeutlicht werden können (vgl. Abbildung 1).

Im Zeitpunkt I bestehe das Kapitalmarktgleichgewicht $\left(\bar{r}^{*}, \mathrm{~K}_{\mathrm{I}}^{*}\right)$ in einer kleinen Region i. Senkt diese Region ihren Steuersatz von $\mathrm{t}^{\mathrm{l}}$ auf $\mathrm{t}^{\mathrm{Il}}$, so verschiebt sich die Nachfragekurve nach rechts. Da das Kapitalangebot vollkommen preiselastisch ist, kann die Anpassung auf dem Markt nicht durch eine Preisanpassung, d.h. durch eine Nettozinssatzerhöhung erfolgen. Das neue Gleichgewicht $\left(\bar{r}^{*}, \mathrm{~K}_{\mathrm{II}}{ }^{*}\right)$ wird ausschließlich durch eine Erhöhung der Kapitalmenge von $K_{I}^{*}$ auf $K_{I I}$ in der Region i erreicht. Umgekehrtes gilt bei einer Erhöhung des Steuersatzes. 


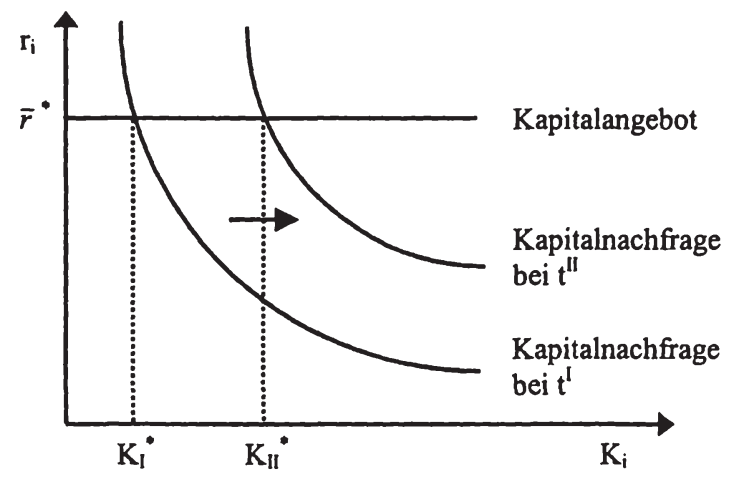

Abbildung 1: Anpassung des Kapitalmarkts eines kleinen Landes

Um von einem Steuerwettbewerb zwischen Regionen sprechen zu können, muss die Wahl des Steuersatzes in einer Region Einfluss ausüben auf die Wahl des Steuersatzes in einer anderen Region. ${ }^{1} \mathrm{Da}$ jedoch die Höhe des Steuersatzes der Region i die Höhe der Kapitalmenge in den Konkurrenzregionen nicht beeinflusst, können die Konkurrenzregionen ohne Beachtung des Steuersatzes der Region i ihren Steuersatz wählen. Somit befinden sich die Regionen nicht in einem Steuerwettbewerb. Das Modell eines kleinen Landes mit vollkommen preiselastischen Kapitalangebot ist insofern ungeeignet, um einen Steuerwettbewerb darzustellen.

\subsection{Exogen vorgegebener Kapitalbestand}

Ein anderes Kapitalmarktmodell geht davon aus, dass die Welt aus zwei Regionen ( $a$ und $b$ ) besteht. In beiden Regionen werden die Produktionsfaktoren Arbeit $(\mathrm{L})$ und Kapital $(\mathrm{K})$ eingesetzt, um das Einheitsgut $(\mathrm{Y})$ herzustellen. Als Produktionsfunktion erhält man folglich: ${ }^{2}$

$$
Y_{a}=f_{a}\left(K_{a}, L_{a}\right)
$$

bzw.

$$
Y_{b}=f_{b}\left(K_{b}, L_{b}\right)
$$

\footnotetext{
' Vgl. Graf (1999, S. 226).

${ }^{2}$ Vgl. Fuest (1997, S. 176).
} 
Das Einheitsgut dient als numéraire mit einem Preis von Eins. ${ }^{1}$ Der Faktor Arbeit sei vollständig immobil. Kapital wird als vollkommen mobil angenommen. ${ }^{2}$ Die Grenzerträge des Kapitals sind positiv und abnehmend. ${ }^{3}$

Jede der beiden Regierungen erzielt Einnahmen durch Steuern auf das Kapital. Die Doppelbesteuerung wird nach dem Quellenlandprinzip vermieden. ${ }^{4}$ Die Analyse wird wiederum sowohl für eine Substanzsteuer als auch für eine Ertragsteuer durchgeführt. Der Einfachheit halber wird ebenfalls unterstellt, dass die Regionen neben den Einnahmen aus Kapitalsteuern weder Einnahmen noch Ausgaben haben, die Einfluss auf den Kapitalmarkt nehmen.

\subsubsection{Gleichgewicht auf dem Kapitalmarkt}

Die Unternehmen treten als Nachfrager auf dem Kapitalmarkt auf. Gelangt eine Substanzsteuer zur Anwendung, so fragen sie in Region a Kapital in Höhe von

$$
K_{a}=f_{z a}\left(r_{a}+t_{a, s}\right)
$$

bzw. in Region b Kapital in Höhe von

$$
K_{b}=f_{z b}\left(r_{b}+t_{b, s}\right)
$$

nach (siehe Gleichung (2.12)). ${ }^{5}$ Erheben die Regierungen eine Ertragsteuer auf das Kapital, so werden die Unternehmen in Region a Kapital in Höhe von

$$
K_{a}=f_{z a}\left(\frac{r_{a}}{\left(1-t_{a, e}\right)}\right)
$$

und die in Region b Kapital in Höhe von

$$
K_{b}=f_{z b}\left(\frac{r_{b}}{\left(1-t_{b, e}\right)}\right)
$$

einsetzen (vgl. Gleichung (2.13)). Die gesamte Kapitalnachfrage der beiden Regionen $\left(\mathrm{K}_{\mathrm{d}}\right)$ ergibt sich aus der Summe der Kapitalnachfragen in den einzelnen Regionen:

$$
K_{d}=K_{a}+K_{b} .
$$

\footnotetext{
${ }^{1}$ Vgl. Wildasin (1988, S. 231), Hensberg (2002, S. 5). vergleiche auch Apolte (1999, S. 24 ff.).

${ }^{3}$ Vgl. Wildasin (1991, S. 397), Hange / Wellisch (1998, S. 317).

${ }^{4} \mathrm{Vgl}$. Roloff et al. (1994, S. 61 ff.), Fuest (1997, S. 176).

${ }^{5}$ Siehe für die Herleitung der Kapitalnachfragefunktion Abschnitt 2.1.1.
}

${ }^{2}$ Vgl. beispielsweise Edwards / Keen (1996, S. 117). Für den Realitätsbezug dieser Annahme 
Unter Berücksichtigung von Gleichung (2.28) bis Gleichung (2.31) zeigt sich, dass die Höhe der gesamten Kapitalnachfrage durch die Steuersätze und die Nettozinssätze festgelegt wird. Sie beläuft sich für den Fall einer Kapitalsubstanzsteuer auf:

$$
K_{d}=f_{z a}\left(r_{a}+t_{a, s}\right)+f_{z b}\left(r_{b}+t_{b, s}\right)
$$

und für den Fall einer Kapitalertragsteuer auf:

$$
K_{d}=f_{z a}\left(\frac{r_{a}}{\left(1-t_{a, e}\right)}\right)+f_{z b}\left(\frac{r_{b}}{\left(1-t_{b, e}\right)}\right) \text {. }
$$

Die Kapitaleigner bieten ihr Kapital in den Regionen an. Sie stellen ihr Kapital dort den Unternehmen zur Verfügung, wo sie die höchste Nettorendite erwirtschaften können. ${ }^{1}$ Es wurde angenommen, dass Kapital vollkommen mobil ist. Daher entsteht ein einheitlicher Nettozinssatz (r), so dass gilt: ${ }^{2}$

$$
r=r_{a}=r_{b} \text {. }
$$

Der Kapitalbestand auf der Welt $(\bar{K})$ sei exogen vorgegeben und fix. ${ }^{3}$ Dieser entspricht dem aggregierten Kapitalangebot $\left(\mathrm{K}_{\mathrm{s}}\right)$ :

$$
\text { (2.36) } K_{s}=\bar{K} \text {. }
$$

Es liegt ein Gleichgewicht auf dem Weltkapitalmarkt vor, wenn bei einem einheitlichen Nettozinssatz (r) das aggregierte Kapitalangebot mit der aggregierten Kapitalnachfrage übereinstimmt: ${ }^{4}$

$$
K_{s}=K_{d} \text {. }
$$

Wird eine Kapitalsubstanzsteuer angewendet, so besteht die Gleichgewichtsbedingung für den Kapitalmarkt daher in:

$$
\text { (2.38) } \quad \bar{K}=f_{z a}\left(r+t_{a, s}\right)+f_{z b}\left(r+t_{b, s}\right)
$$

(vgl. Gleichung (2.33) und Gleichung (2.35) bis Gleichung (2.37)). Wird hingegen eine Kapitalertragsteuer erhoben, dann ergibt sich folgende Gleichgewichtsbedingung:

$$
\bar{K}=f_{z a}\left(\frac{r}{\left(1-t_{a, e}\right)}\right)+f_{z b}\left(\frac{r}{\left(1-t_{b, e}\right)}\right)
$$

(vgl. Gleichung (2.34) bis Gleichung (2.37)).

\footnotetext{
${ }^{1}$ Vgl. Hange / Wellisch (1998, S. 317), Hensberg (2002, S. 7).

${ }^{2}$ Vgl. Wildasin (1991, S. 397), Edwards / Keen (1996, S. 117).

${ }^{3}$ Vgl. Wildasin (1991, S. 397), Fuest (2000, S. 360).

${ }^{4}$ Vgl. Hange / Wellisch (1998, S. 317).
} 


\subsubsection{Auswirkung einer Änderung des Steuersatzes}

Für eine Analyse der Nettozinssatzänderung als Reaktion auf eine marginale Veränderung des Steuersatzes in einer der Regionen ist die Ableitung von Gleichung (2.38) bzw. Gleichung (2.39) nach dem Steuersatz zu ermitteln. Im Fall einer Substanzsteuer führt eine marginale Veränderung des Steuersatzes von Region a zu einer Änderung des Nettozinssatzes in Höhe von:'

$$
\frac{\partial r}{\partial t_{a, s}}=\frac{-1}{\left(1+\frac{f_{z b z b}\left(r+t_{b, s}\right)}{f_{z a z a}\left(r+t_{a, s}\right)}\right)} \text {. }
$$

Entsprechend erhält man für eine marginale Änderung des Steuersatzes der Region b:

$$
\frac{\partial r}{\partial t_{b, s}}=\frac{-1}{\left(1+\frac{f_{z a z a}\left(r+t_{a, s}\right)}{f_{z b z b}\left(r+t_{b, s}\right)}\right)} .
$$

Im Fall einer Kapitalertragsteuer bewirkt eine marginale Änderung des Steuersatzes der Region a folgende Änderung des Nettozinssatzes: ${ }^{2}$

$$
\frac{\partial r}{\partial t_{a, e}}=\frac{-1}{\left(1-t_{a, e}\right)} \cdot \frac{r}{\left.1+\frac{\left(1-t_{a, e}\right) \cdot f_{z b z b}\left(\frac{r}{\left(1-t_{b, e}\right)}\right)}{\left(1-t_{b, e}\right) \cdot f_{z a z a}\left(\frac{r}{\left(1-t_{a, e}\right)}\right)}\right)}
$$

Ebenso ergibt sich für Region $b$ :

$$
\frac{\partial r}{\partial t_{b, e}}=\frac{-1}{\left(1-t_{b, e}\right)} \cdot \frac{r}{\left.1+\frac{\left(1-t_{b, e}\right) \cdot f_{z a z a}\left(\frac{r}{\left(1-t_{a, e}\right)}\right)}{\left(1-t_{a, e}\right) \cdot f_{z b z b}\left(\frac{r}{\left(1-t_{b, e}\right)}\right)}\right)} .
$$

Berücksichtigt man Gleichung (2.16), so zeigt sich, dass für alle vier Fälle

${ }_{1}^{1}$ Siehe Anhang la für die Berechnung von Gleichung (2.40).

${ }^{2}$ Für die Berechnung von Gleichung (2.42) siehe Anhang $1 \mathrm{~b}$. 
(2.44) $\frac{\partial r}{\partial t}<0$

gilt. Wird also der Kapitalsteuersatz in einer Region erhöht, dann sinkt die Nettorendite und umgekehrt. Im Gegensatz zu dem in Abschnitt 2.1 diskutierten Modell verursacht eine Steuersatzänderung im Rahmen dieses Modells stets eine Anpassung des Nettozinssatzes.

Der Kapitalmarkt findet aber nicht nur über den soeben skizzierten Preisanpassungsprozess sein Gleichgewicht. Steuersatzänderungen lösen im Rahmen dieses Modells immer auch Mengenanpassungsprozesse aus, d.h. sie führen stets zu einer Kapitalrelokation. Nach Gleichung (2.28) und Gleichung (2.29) entspricht im Fall der Substanzsteuer die Kapitalmenge, die in Region a nachgefragt wird:

(2.45) $\quad K_{a}=f_{z a}\left(r_{a}+t_{a, s}\right)$

bzw. die, die in Region b eingesetzt wird:

(2.46) $\quad K_{b}=f_{z b}\left(r_{b}+t_{b, s}\right)$.

Für den Fall einer Kapitalertragsteuer ergibt sich die Kapitalmenge der Region a wie folgt:

$$
K_{a}=f_{z a}\left(\frac{r_{a}}{\left(1-t_{a, e}\right)}\right)
$$

bzw. die der Region b als:

$$
K_{b}=f_{z b}\left(\frac{r_{b}}{\left(1-t_{b, e}\right)}\right)
$$

(siehe Gleichung (2.30) und Gleichung (2.31)). Die Änderung der Kapitalmenge in der Region a bei einer marginalen Änderung des Steuersatzes in derselben Region beläuft sich im Fall der Substanzsteuer daher auf:'

$$
\frac{\partial K_{a}}{\partial t_{a, s}}=\frac{1}{\left(\frac{1}{f_{z b z b}\left(r+t_{b, s}\right)}+\frac{1}{f_{z a z a}\left(r+t_{a, s}\right)}\right)}
$$

und im Fall der Ertragsteuer auf: ${ }^{2}$

${ }^{1}$ Die Berechnungen für Gleichung (2.49) sind in Anhang 2a aufgeführt.

${ }^{2} \mathrm{Vgl}$. für die Berechnungen, die zu Gleichung (2.50) führen, Anhang $2 \mathrm{~b}$. 


$$
\frac{\partial K_{a}}{\partial t_{a, e}}=\frac{r}{\left(1-t_{a, e}\right)} \cdot \frac{1}{\left(\frac{\left(1-t_{b, e}\right)}{f_{z b z b}\left(\frac{r}{\left(1-t_{b, e}\right)}\right)}+\frac{\left(1-t_{a, e}\right)}{f_{z a z a}\left(\frac{r}{\left(1-t_{a, e}\right)}\right)}\right)} .
$$

Entsprechend erhält man für den Fall einer Kapitalsubstanzsteuer bei einer marginalen Änderung des Steuersatzes der Region b eine Änderung der Kapitalmenge in Region b in Höhe von:

$$
\frac{\partial K_{b}}{\partial t_{b, s}}=\frac{1}{\left(\frac{1}{f_{z b z b}\left(r+t_{b, s}\right)}+\frac{1}{f_{z a z a}\left(r+t_{a, s}\right)}\right)}
$$

und für den Fall einer Kapitalertragsteuer eine Änderung in Höhe von:

$$
\frac{\partial K_{b}}{\partial t_{b, e}}=\frac{r}{\left(1-t_{b, e}\right)} \cdot \frac{1}{\left(\frac{\left(1-t_{b, e}\right)}{f_{z b z b}\left(\frac{r}{\left(1-t_{b, e}\right)}\right)}+\frac{\left(1-t_{a, e}\right)}{f_{z a z a}\left(\frac{r}{\left(1-t_{a, e}\right)}\right)}\right)} .
$$

In allen vier zuvor skizzierten Fällen gilt:

$$
\text { (2.53) } \frac{\partial K_{i}}{\partial t_{i}}<0 \text {. }
$$

Die Erhöhung (Senkung) des Steuersatzes in einer Region (i) führt c.p. bei eben dieser Region also zum Kapitalabfluss (Kapitalzufluss).

$\mathrm{Da}$ der Kapitalbestand in der Welt als exogen gegeben und fix angenommen wurde, entspricht der Kapitalabfluss der einen Region genau dem Kapitalzufluss der anderen Region und umgekehrt. Insofern liegt in den beiden betrachteten Fällen (Fall der Substanzsteuer und Fall der Ertragsteuer)

$$
\frac{\partial K_{b}}{\partial t_{a}}=-\frac{\partial K_{a}}{\partial t_{a}}>0
$$

bzw.

$$
\frac{\partial K_{a}}{\partial t_{b}}=-\frac{\partial K_{b}}{\partial t_{b}}>0
$$


vor. ' Senkt also eine der Regionen ihren Steuersatz, dann fließt unter sonst unveränderten Umständen aus der Konkurrenzregion Kapital ab und umgekehrt. Eine Region muss also im Rahmen dieses Modells stets der Konkurrenzregion Kapital abziehen, um selbst zusätzliches Kapital attrahieren zu können. Die Regionen stehen somit im direkten Wettbewerb um Kapital. Im Gegensatz zu dem in Abschnitt 2.1 vorgestellten Modell bleibt die Wirkung der Steuersatzänderung einer Region nicht auf diese Region beschränkt. Insofern können die Reaktionen auf dem Kapitalmarkt bezüglich einer Steuersatzänderung einer der Regionen auch nicht für eine Region alleine dargestellt werden. Der Kapitalmarkt beider Regionen ist als Einheit mit Interdependenzen zu verstehen und kann daher auch nur insgesamt in einer Grafik veranschaulicht werden (vgl. Abbildung 2).

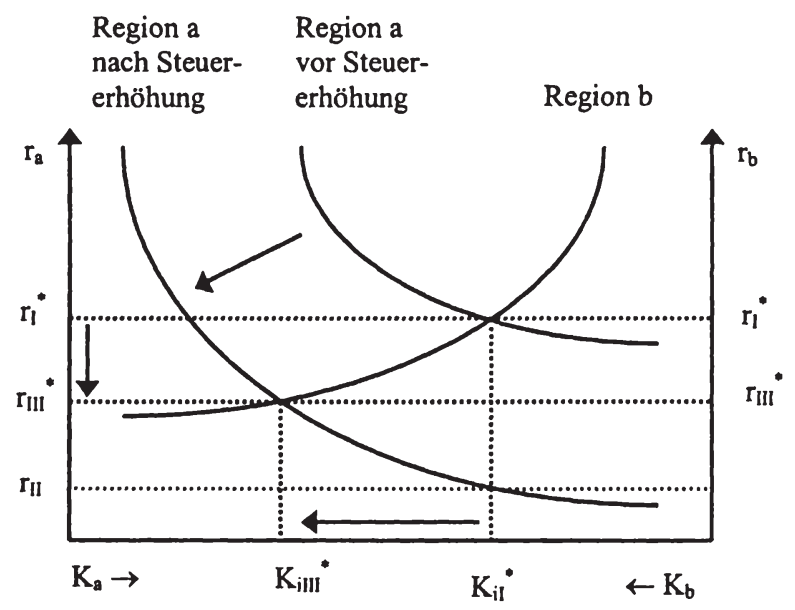

Abbildung 2: Anpassungen des Kapitalmarkts an eine Steuersatzänderung

$\mathrm{Zu}$ Anfang befindet sich der Kapitalmarkt im Gleichgewicht $\left(\mathrm{r}_{1}^{*}, \mathrm{~K}_{\mathrm{il}}{ }^{\circ}\right)$. Region a erhöht nun einmalig ihren Kapitalsteuersatz, wodurch sich die Nachfragekurve von Region a nach links verschiebt. Es liegt ein Ungleichgewicht auf dem Kapitalmarkt vor, bei dem die Nettorendite in Region a $\left(\mathrm{r}_{\mathrm{II}}\right)$ geringer ausfällt als die

\footnotetext{
' Der exakte, mathematische Beweis furr diese Behauptung ist fur den Fall der Substanzsteuer in Anhang 3a und für den Fall der Ertragsteuer in Anhang $3 \mathrm{~b}$ geführt.
} 
in Region $b\left(r_{I}^{*}\right)$. Daraufhin verlagern die Kapitaleigner von Region a in Region b Kapital, weil sie dort eine höhere Nettorendite erwirtschaften können. Dadurch fällt die Bruttorendite in Region b und die in Region a steigt an, was bei jetzt unveränderten Steuersätzen zu einer entsprechenden Änderung der Nettorenditen führt. Das neue Gleichgewicht $\left(\mathrm{r}_{\mathrm{III}}{ }^{*}, \mathrm{~K}_{\mathrm{iIII}}{ }^{*}\right)$ wird somit sowohl durch Kapitalrelokation als auch durch Anpassung des Nettozinssatzes erreicht.

Ändert demnach eine Region ihren Steuersatz, so ist die Wirkung nicht nur auf diese Region begrenzt, sondern entfaltet sich auch in der Konkurrenzregion. Möchte also eine Regierung ihren Steuersatz variieren, so hat sie zu bedenken, dass ihre Steuersatzvariation auch Auswirkungen auf die Konkurrenzregionen hat. Diese werden ihre Steuerpolitik entsprechend anpassen, was wiederum Rückwirkungen auf die eigene Region hat. Insofern kann die einzelne Region ihren Steuersatz nicht ändern, ohne dabei die steuerpolitischen Reaktionen in der Konkurrenzregion zu beachten. Dann aber beeinflusst die Wahl des Steuersatzes einer Region die Wahl des Steuersatzes der anderen Region, so dass ein Steuerwettbewerb vorliegt. Dieses Modell ist demnach in der Lage, einen Steuerwettbewerb um Kapital abzubilden.

\subsection{Zusammenfassung}

Abschließend lässt sich also festhalten, dass nur das zweite der beiden betrachteten Modelle einen Steuerwettbewerb um Kapital darzustellen vermag.

In dem ersten Modell, das von einem vollkommen preiselastischen Kapitalangebot auf dem Kapitalweltmarkt ausgeht, erfolgt eine Anpassung an eine Steuersatzänderung einer Region ausschließlich über eine Änderung der Kapitalmenge in eben dieser Region. Durch eine Steuersatzänderung wird keine Änderung des Nettozinssatzes ausgelöst. Daher führt eine Steuersatzvariation einer Region i auch nicht zu einer Kapitalmengenänderung der Konkurrenzregionen. Die Konkurrenzregion kann daher unabhängig von der Steuerpolitik der Region $\mathrm{i}$ ihren Steuersatz wählen, weshalb nicht von einem Steuerwettbewerb gesprochen werden kann.

In dem zweiten Modell, das einen exogen vorgegebenen Kapitalbestand unterstellt, wirkt sich hingegen eine Steuersatzänderung weitaus anders aus. So bewirkt eine Änderung des Steuersatzes in einer der Regionen eine Anpassung des 
Nettozinssatzes. Darüber hinaus führt sie zu einer Kapitalrelokation zwischen beiden Regionen. Die eine Region muss also Kapital verlieren, damit in der anderen Region zusätzliches Kapital eingesetzt werden kann. Zwischen den Regionen herrscht demnach ein Wettbewerb um Kapital. Insofern muss allerdings auch die eine Region bei ihrer Entscheidung bezüglich des Steuersatzes die Steuerpolitik der anderen Region berücksichtigen, da die Wirkung der Steuerpolitik nicht auf eine Region beschränkt ist. Dieses Modell ist demnach geeignet, um Steuerwettbewerb abzubilden.

Der folgende Teil der Arbeit befasst sich mit Fragestellungen zum fiskalischen Wettbewerb um Kapital. Fiskalischer Wettbewerb besteht zum einen in Steuerwettbewerb zum anderen aber auch in Ausgabenwettbewerb. Wie gezeigt wurde, lässt sich Steuerwettbewerb nur mit Hilfe des Kapitalmarktmodells, welches einen exogen vorgegebenen Kapitalbestand unterstellt, und nicht durch das Modell, welches ein vollkommen preiselastisches Kapitalangebot annimmt, darstellen. Diese Erkenntnis ist auf den Ausgabenwettbewerb übertragbar, da eine Analyse des Ausgabenwettbewerbs zu gleichen Ergebnissen führt wie die hier durchgeführte Analyse des Steuerwettbewerbs. Aus diesem Grund werden sich die folgenden Untersuchungen auf das Kapitalmarktmodell stützen, welches einen exogen vorgegebenen Kapitalbestand unterstellt und welches in Abschnitt 2.2 vorgestellt wurde.

\section{Wirkung der fiskalpolitischen Instrumente auf dem Kapitalmarkt im Wettbewerb}

Aktuelle Themen wie die Neugestaltung des deutschen Finanzausgleichs und die Gestaltung der Europäischen Union geben immer wieder Anlass, sich mit dem Thema „Standortwettbewerb“ auseinander zu setzen. Dementsprechend groß ist die Zahl der Aufsätze, die sich mit diesem Thema befassen. In der Regel wird in diesen berücksichtigt, dass die Regierungen sowohl Einnahmen in Form von Steuern erzielen als auch Ausgaben für öffentliche Güter tätigen.' Besondere Beachtung wird häufig der Wirkung von Steuern auf dem Kapitalmarkt ge-

\footnotetext{
I Vgl. beispielsweise Oates / Schwab (1988), Edwards / Keen (1996), Sinn (1997), Amold / Fuest (1999).
} 
schenkt. ${ }^{1}$ Ein entsprechendes Interesse findet die Wirkung von öffentlichen Zwischenprodukten auf dem Kapitalmarkt hingegen nicht. So ist es auch nicht verwunderlich, dass die derzeitige Regierung in erster Linie durch Steuersenkungen Anreize für Kapital geben möchte. ${ }^{2}$ Daher hat sie, um die Wettbewerbsfähigkeit Deutschlands zu sichern, das Steuerentlastungsgesetz 1999/2000/2002 beschlossen, welches durch Ausgabeneinsparungen gegenfinanziert werden soll. ${ }^{3}$ Inwiefern eine derartige Politik geeignet sein kann, zusätzliches Kapital in die Region zu locken, soll im Weiteren überprüft werden. Im Gegensatz zur Literatur zum Standortwettbewerb wird dabei insbesondere die Rolle von öffentlichen Zwischenprodukten auf dem Kapitalmarkt thematisiert.

Das Kapitel ist wie folgt strukturiert: In Abschnitt 3.1 wird ein Grundmodell fiskalischen Standortwettbewerbs skizziert, wobei die den Regierungen zur Verfügung stehenden fiskalpolitischen Instrumente und das Entstehen eines Gleichgewichtes auf dem Kapitalmarkt erläutert werden. Auf Basis dieses Grundmodells kann dann in Abschnitt 3.2 sowohl die Wirkung einer Steuersatzänderung als auch die Wirkung einer Änderung der Bereitstellungsmenge an öffentlichem Zwischenprodukt auf dem Kapitalmarkt analysiert werden. In Abschnitt 3.3 wird die Wirkung beider Instrumente zusammen untersucht. Dabei wird insbesondere das Ausmaß der Substituierbarkeit der fiskalpolitischen Instrumente festgestellt und ein Wert definiert, der das Niveau beider fiskalpolitischer Instrumente zusammengefasst wiedergibt. In Abschnitt 3.4 werden dann die Probleme, die beim Wettbewerb um Kapital auftreten können, thematisiert. Abschnitt 3.5 enthält eine Zusammenfassung der Ergebnisse.

\subsection{Grundmodell des fiskalischen Standortwettbewerbs}

Im Mittelpunkt der Betrachtung steht eine Föderation, die aus zwei Regionen (a und b) besteht. In jeder dieser Regionen (i) gelangen die beiden privaten Primärfaktoren Arbeit (L) und Kapital (K) zum Einsatz. Es wird angenommen, dass

\footnotetext{
${ }^{1}$ Vgl. zum Beispiel DePater / Myers (1994), Hange / Wellisch (1998), Homburg (1999), Apolte (2001).

${ }^{2}$ Vgl. BT-Drucks. 14/2683, S. 93.

${ }^{3}$ Vgl. BT-Drucks. 14/2683, S. 92. 
das Kapital vollständig mobil ist. ${ }^{\prime}$ Arbeit wird als vollkommen immobil unterstellt. In jeder Region produzieren Unternehmen, deren Anzahl exogen vorgegeben ist, ein Einheitsgut (Y). Dieses Einheitsgut dient als numéraire mit einem Preis von Eins. ${ }^{2}$

\subsubsection{Fiskalpolitische Instrumente}

Jede Region wird von einer Regierung regiert, die mit Hilfe der fiskalpolitischen Instrumente ihre Ziele verfolgt. Um die Gültigkeit der Analyse nicht weiter einzuschränken, wird die Zielfunktion der Regierungen nicht definiert. Somit bleiben die folgende Untersuchung und ihre Ergebnisse sowohl für Ansätze die eine wohlwollende Regierung als auch für solche die eine eigennützige Regierung modellieren zugänglich. Als fiskalpolitische Instrumente, die die Regierung einsetzen kann, stehen eine Steuer und ein öffentliches Gut zur Verfügung.

Bei der Steuer handelt es sich um eine Kapitalertragsteuer $\left(T_{i}\right)$. Die eventuell auftretende Doppelbesteuerung wird nach dem Quellenlandprinzip vermieden, ${ }^{3}$ so dass die Besteuerung des Kapitals in der Region erfolgt, in der das Kapital eingesetzt wird und daraus Erträge erwirtschaftet werden. Die Einnahmen, die die jeweilige Regierung durch die Kapitalertragsteuer erzielt, belaufen sich daher auf das Produkt aus Steuersatz $\left(t_{i}\right)$, dem für die Region gültigen Bruttozinssatz $\left(z_{i}\right)$ und der Menge an in der Region eingesetztem Kapital $\left(\mathrm{K}_{\mathrm{i}}\right)$ :

$$
T_{i}=t_{i} \cdot z_{i} \cdot K_{i} \text {. }
$$

Erhebt die Regierung diese Kapitalertragsteuer, so ist zwischen dem Bruttozinssatz $\left(\mathrm{z}_{\mathrm{i}}\right)$ und dem Nettozinssatz $\left(\mathrm{r}_{\mathrm{i}}\right)$ zu unterscheiden. Den Nettoertrag aus Kapital $\left(r_{i} \cdot K_{i}\right)$ erhält man, indem der Bruttoertrag aus Kapital $\left(z_{i} \cdot K_{i}\right)$ um die zu leistenden Steuern $\left(\mathrm{T}_{\mathrm{i}}\right)$ verringert wird:

$$
r_{i} \cdot K_{i}=z_{i} \cdot K_{i}-t_{i} \cdot z_{i} \cdot K_{i} \text {. }
$$

Demnach entspricht der Nettozinssatz dem Produkt aus dem Bruttozinssatz und dem Steuerfaktor $\left(1-t_{i}\right)$ :

\footnotetext{
${ }^{\prime}$ Vgl. Oates / Schwab (1988, S. 337), Edwards / Keen (1996, S. 117), Sinn (1997, S. 14 und 18), Rauscher (1998, S. 61), Tausch (1998, S. 330). Zum Realitătsbezug dieser Annahme siehe auch Apolte (1999, S. 24 ff.).

${ }^{2}$ Vgl. Wildasin (1988, S. 231), Tausch (1998, S. 330).

${ }^{3}$ Vgl. Roloff et al. (1994, S. 61 ff.), Edwards / Keen (1996, S. 117), Fuest (1997, S. 176). 


$$
r_{i}=z_{i} \cdot\left(1-t_{i}\right)
$$

bzw. der Bruttozinssatz dem Quotienten aus Nettozinssatz und Steuerfaktor:

$$
z_{i}=\frac{r_{i}}{\left(1-t_{i}\right)} \text {. }
$$

Das öffentliche Gut, welches die Regierung bereitstellt, ist kein Konsumgut, sondern ein Zwischenprodukt, welches kostenlos in die Produktion der Unternehmen einfließt. Dieses Gut ist ein lokal öffentliches Gut, so dass die Nutzung des öffentlichen Gutes durch gebietsfremde Unternehmen nicht möglich ist. ${ }^{1}$ Interregionale Externalitäten, so genannte „spillovers“, sind somit ausgeschlossen. ${ }^{2}$ Darüber hinaus handelt es sich bei diesem öffentlichen Zwischenprodukt um ein rein öffentliches Gut im Sinne von Samuelson, das sich durch NichtRivalität im Konsum auszeichnet. ${ }^{3}$ Insofern liegt ein „factor-augmenting public input" vor. ${ }^{4}$ Daher ist die Berücksichtigung von Ballungskosten nicht notwendig, weil sich diese aufgrund der fehlenden Nutzungsrivalität auf Null belaufen. ${ }^{5}$ Das öffentliche Zwischenprodukt führt zu einem Anstieg des Outputs des Einheitsgutes und wirkt insofern produktivitätssteigernd. ${ }^{6}$ Des weiteren ist es Hicksneutral, weshalb es die gleiche Wirkung auf beide privaten Primärfaktoren hat. ${ }^{7}$ Die Wirkung des öffentlichen Zwischenprodukts im Produktionsprozess kann durch einen Effizienzparameter (E) beschrieben werden. ${ }^{8}$ Dieser Parameter hängt von der Höhe der bereitgestellten Menge an öffentlichem Input ab:

$$
E_{i}=E_{i}\left(G_{i}\right) \text {. }
$$

Die Wirkung einer bestimmten Menge an öffentlichem Zwischenprodukt ist in allen Regionen gleich, so dass auf den Index des Effizienzparameters verzichtet werden kann. Wenn das öffentliche Zwischenprodukt nicht bereitgestellt wird, dann nimmt er den Wert Eins an:

$$
E(0)=1 \text {. }
$$

Mit steigendem Einsatz des Zwischenprodukts steigt der Parameter derart an, dass

\footnotetext{
${ }^{1}$ Vgl. Richter / Wellisch (1993, S. 436 und 438).

${ }^{2}$ Vgl. Hange / Wellisch (1998, S. 318).

${ }^{3} \mathrm{Vgl}$. Samuelson (1954).

${ }^{4}$ Vgl. Kaizuka (1965, S. 118 ff.), Tausch (1998, S. 331), Colombier (2001, S. 43 ff.).

${ }^{5}$ Vgl. Sinn (1997, S. 17), Tausch (1998, S. 335).

${ }^{6} \mathrm{Vgl}$. Tausch (1998, S. 331).

${ }^{7}$ Vgl. Colombier (2001, S. 45).

${ }^{8}$ Vgl. Tausch (1998, S. 331), Roloff (1999, S. 50; 2001, S. 116 f.). 


$$
\frac{d E\left(G_{i}\right)}{d G_{i}}>0
$$

und

$$
\frac{d^{2} E\left(G_{i}\right)}{d G_{i}^{2}}<0
$$

gilt.

\subsubsection{Der Kapitalmarkt}

Auf dem Kapitalmarkt treten die Unternehmen als Nachfrager auf, da sie neben der Arbeit und dem öffentlichen Zwischenprodukt Kapital einsetzen, um das Einheitsgut zu produzieren. Die Branchenproduktionsfunktion der jeweiligen Region i entspricht daher:'

$$
Y_{i}=E\left(G_{i}\right) \cdot f_{i}\left(K_{i}, L_{i}\right) .
$$

Die Grenzproduktivität des Kapitals ist positiv und abnehmend: ${ }^{2}$

$$
\begin{aligned}
& \frac{\partial f_{i}\left(K_{i}, L_{i}\right)}{\partial K_{i}} \equiv f_{K i}\left(K_{i}\right)>0, \\
& \frac{\partial^{2} f_{i}\left(K_{i}, L_{i}\right)}{\partial K_{i}^{2}}=f_{K i K_{i}}\left(K_{i}\right)<0 .
\end{aligned}
$$

Das Ziel der Unternehmen liegt darin, ihren Gewinn $\left(\Pi_{i}\right)$ zu maximieren. Diesen erhält man durch Subtraktion der Ausgaben, die aus der Entlohnung der beiden Primärfaktoren - Kapital und Arbeit - entstehen, von den Einnahmen, die sich aufgrund eines Preises von Eins auf die Outputmenge belaufen. Die Gewinnfunktion entspricht demnach: ${ }^{3}$

$$
\Pi_{i}=E\left(G_{i}\right) \cdot f_{i}\left(K_{i}, L_{i}\right)-z_{i} \cdot K_{i}-w_{i} \cdot L_{i},
$$

\footnotetext{
'Vgl. Michael (1995, S. 74), Colombier (2001, S. 45).

2 Vgl. Zodrow Mieszkowski (1986, S. 358), Wildasin (1991, S. 397), Hange / Wellisch (1998, S. 317), Tausch (1998, S. 331).

${ }^{3}$ Vgl. Arnold / Fuest (1999, S. 166), Homburg (1999, S. 3), Kollintzas / Philippopoulos / Vassilatos (2000, S. 6), Apolte (2001, S. 361).
} 
wobei $w_{i}$ den Bruttolohnsatz und $z_{i}$ den Bruttozinssatz der jeweiligen Region darstellen. Die Unternehmen erreichen ihr Gewinnmaximum, wenn u.a. die Grenzproduktivität des Kapitals mit dem Bruttozinssatz übereinstimmt: ${ }^{1}$

$$
E\left(G_{i}\right) \cdot f_{K i}\left(K_{i}\right)=z_{i} \text {. }
$$

Berücksichtigt man den in Gleichung (3.4) dargestellten Zusammenhang zwischen Brutto- und Nettozinssatz, dann kann diese Bedingung auch in Abhängigkeit des Nettozinssatzes ausgedrückt werden:

$$
E\left(G_{i}\right) \cdot f_{K i}\left(K_{i}\right)=\frac{r_{i}}{\left(1-t_{i}\right)} .
$$

Gleichung (3.14) enthält implizit die Höhe der in der jeweiligen Region nachgefragten Kapitalmenge. Definiert man $f_{\mathrm{P} i}$ als die Umkehrfunktion von $f_{K i}$, so kann die Höhe an nachgefragtem Kapital in einer Region explizit bestimmt werden:

$$
K_{i}=f_{\mathrm{P} i}\left(\frac{r_{i}}{E\left(G_{i}\right) \cdot\left(1-t_{i}\right)}\right) .
$$

Da Kapital eine nicht-negative Größe ist, liegt

$$
f_{\mathrm{P} i}\left(\frac{r_{i}}{E\left(G_{i}\right) \cdot\left(1-t_{i}\right)}\right) \geq 0
$$

vor. Des weiteren gilt, dass die Ableitung einer Umkehrfunktion genau mit dem Reziprok der Ableitung der Originalfunktion übereinstimmt: ${ }^{2}$

$$
f_{\mathrm{PiP} i}=\frac{1}{f_{K i K i}} \text {. }
$$

Aus Gleichung (3.11) kann somit gefolgert werden, dass

$$
f_{\mathrm{P} i P_{i}}\left(\frac{r_{i}}{E\left(G_{i}\right) \cdot\left(1-t_{i}\right)}\right)<0
$$

gültig ist. Der Zusammenhang zwischen dem Nettozinssatz und der Kapitalnachfrage in einer Region ist also negativ. Somit ergibt sich c.p. eine zunehmende (abnehmende) Kapitalnachfrage bei sinkendem (steigendem) Nettozinssatz. Dieser Zusammenhang ist in Abbildung 3 illustriert, in der die Kapitalnachfragefunktion einer Region dargestellt ist.

\footnotetext{
'Vgl. Fuest (1995, S. 481), Hange / Wellisch (1998, S. 317), Apolte (2001, S. 361).

${ }^{2}$ Vgl. Chiang (1984, S. 173).
} 


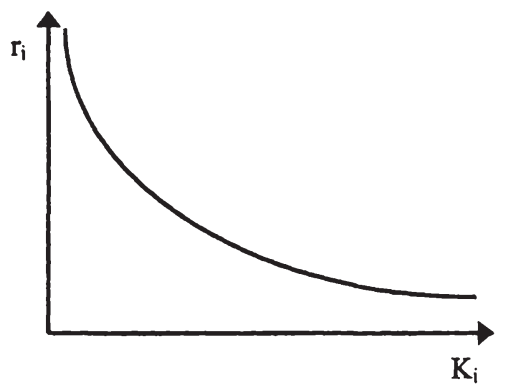

Abbildung 3: Kapitalnachfragefunktion der i-ten Region

Die Kapitalnachfrage der gesamten Föderation $\left(\mathrm{K}_{\mathrm{d}}\right)$ erhält man, indem die Kapitalnachfrage der Region a und die der Region $\mathrm{b}$ addiert wird:

(3.19) $K_{d}=K_{a}+K_{b}$.

Nach Gleichung (3.15) wird deren Höhe durch die Mengen an von den beiden Regionen bereitgestelltem öffentlichen Zwischenprodukt, durch die Kapitalertragsteuersätze beider Regionen und durch die Nettozinssätze festgelegt:

$$
K_{d}=f_{\mathrm{Pa}}\left(\frac{r_{a}}{E\left(G_{a}\right) \cdot\left(1-t_{a}\right)}\right)+f_{\mathrm{P} b}\left(\frac{r_{b}}{E\left(G_{b}\right) \cdot\left(1-t_{b}\right)}\right) .
$$

Der Kapitalbestand der Föderation $(\bar{K})$, der dem aggregierten Kapitalangebot $\left(K_{s}\right)$ entspricht, ist exogen vorgegeben und fix: ${ }^{1}$

$$
K_{s}=\bar{K} \text {. }
$$

Da das Kapital vollkommen mobil ist, stellen die Kapitaleigner der Föderation ihr Kapital dort zur Verfügung, wo sie die höchste Nettorendite erzielen können. ${ }^{2}$ Somit bildet sich eine Nettorendite $(r)$ in der Föderation, die für alle Regionen gleich hoch ist: ${ }^{3}$

$$
r=r_{i} \text {. }
$$

' Vgl. Zodrow / Mieszkowski (1986, S. 358), Oates / Schwab (1988, S. 337), Wildasin (1991, S. 397), Fuest (2000, S. 360).

${ }^{2}$ Vgl. Oates / Schwab (1988, S. 337).

${ }^{3}$ Vgl. Zodrow / Mieszkowski (1986, S. 358), Wildasin (1991, S. 397), Edwards / Keen (1996, S. 117), Apolte (2001, S. 361). 
Der Kapitalmarkt der Föderation ist im Gleichgewicht, wenn bei einem einheitlichen Nettozinssatz (r) die aggregierte Kapitalnachfrage mit dem aggregierten Kapitalangebot übereinstimmt: ${ }^{1}$

$$
K_{s}=K_{d} \text {. }
$$

Setzt man Gleichung (3.20) und Gleichung (3.21) in Gleichung (3.23) ein, so erhält man - unter Berücksichtigung von Gleichung (3.22) - als Gleichgewichtsbedingung für den Kapitalmarkt: ${ }^{2}$

$$
\bar{K}=f_{\mathrm{P} a}\left(\frac{r}{E\left(G_{a}\right) \cdot\left(1-t_{a}\right)}\right)+f_{\mathrm{Pb}}\left(\frac{r}{E\left(G_{b}\right) \cdot\left(1-t_{b}\right)}\right) .
$$

Dieses Gleichgewicht ist grafisch in Abbildung 4 veranschaulicht:

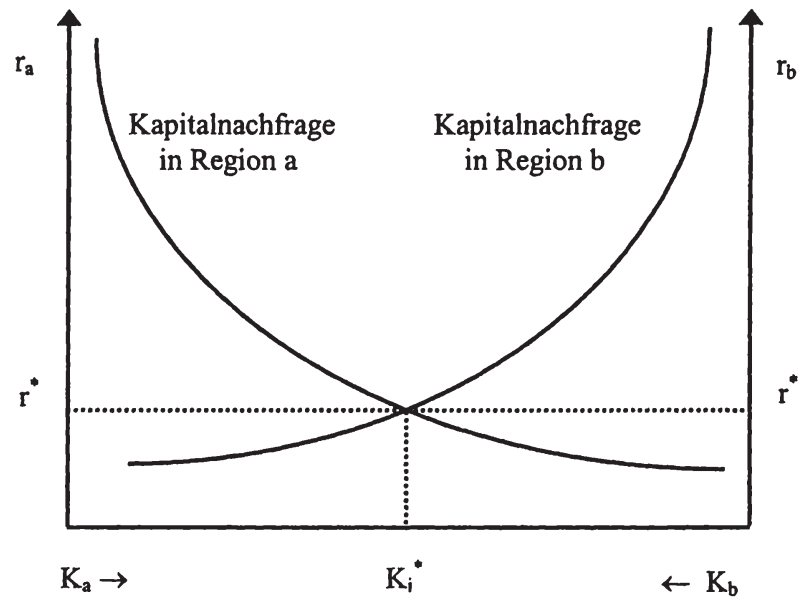

Abbildung 4: Gleichgewicht auf dem Kapitalmarkt

${ }^{1}$ Vgl. Hange / Wellisch (1998, S. 317).

${ }^{2}$ In der Literatur findet man auch folgende Art der Gleichgewichtsbedingung:

$$
\left(1-t_{a}\right) \cdot E\left(G_{a}\right) \cdot f_{K a}\left(K_{a}, L_{a}\right)=\left(1-t_{b}\right) \cdot E\left(G_{b}\right) \cdot f_{K b}\left(\bar{K}-K_{a}, L_{b}\right)
$$

(Vgl. beispielsweise Arnold / Fuest (1999, S. 167). Diese entspricht inhaltlich der in dieser Arbeit verwendeten Gleichgewichtsbedingung. Sie unterscheiden sich also nur in der Schreibweise, da die in der Literatur teilweise verwendete Bedingung explizit die Kapitalmengen der beiden Regionen aufführt und implizit den gleichgewichtigen Nettozinssatz beinhaltet, wohingegen die in dieser Arbeit verwendete Gleichgewichtsbedingung explizit den Nettozinssatz aufführt und implizit die Kapitalmengen der Regionen beinhaltet. Die Schreibweise dieser Arbeit wurde gewählt, weil man bei ihr explizit die Angebots- und Nachfragepläne in der Föderation erkennen kann. 
Im Gleichgewicht entspricht die Summe des in beiden Regionen nachgefragten Kapitals $\left(\mathrm{K}_{\mathrm{i}}^{*}\right)$ dem in der Föderation insgesamt vorhandenen Kapitalbestand $(\bar{K})$. Dieses Gleichgewicht wird durch einen entsprechenden Nettozinssatz ( $\left.\mathrm{r}^{*}\right)$ erreicht.

\subsection{Wirkung der einzelnen fiskalpolitischen Instrumente}

Die Regierungen können die Höhe der von den Regionen attrahierten Kapitalmengen sowie die Höhe des Nettozinssatzes durch ihre fiskalpolitischen Instrumente beeinflussen. Im Rahmen des hier skizzierten Modells kann dies zum einen durch eine Änderung des Kapitalertragsteuersatzes geschehen. Zum anderen wirkt eine Veränderung der bereitgestellten Menge an öffentlichem Input auf die Höhe der attrahierten Kapitalmengen und auf die des Nettozinssatzes. Die folgende Analyse wird nur am Beispiel von Region a vorgenommen. Für Region b gelten die Ergebnisse entsprechend.

\subsubsection{Wirkung der Steuer}

Variiert Regierung a den Kapitalertragsteuersatz, so verändert sich c.p. sowohl der Nettozinssatz als auch die Kapitalverteilung innerhalb der Föderation. Die Veränderung des Nettozinssatzes kann durch Ableitung desselben nach dem Steuersatz $\left(t_{a}\right)$ ermittelt werden. Aus der Kapitalmarktgleichgewichtsbedingung (siehe Gleichung (3.24)) lässt sich bei einer marginalen Änderung des Steuersatzes von Region a die Änderung des Nettozinssatzes in Höhe von'

$$
\frac{\partial r}{\partial t_{a}}=\frac{-1}{\left(1-t_{a}\right)} \cdot \frac{r}{\left(1+\frac{E\left(G_{a}\right) \cdot\left(1-t_{a}\right) \cdot f_{K a K a}\left(K_{a}\right)}{E\left(G_{b}\right) \cdot\left(1-t_{b}\right) \cdot f_{K b K b}\left(K_{b}\right)}\right)}
$$

bestimmen. Der Zusammenhang zwischen Nettozinssatz und Steuersatz ist somit negativ:

$$
\frac{\partial r}{\partial t_{a}}<0
$$

\footnotetext{
${ }^{1}$ Für die Berechnung von Gleichung (3.25) siehe Anhang 4a. 
Erhöht also eine Regierung den Kapitalertragsteuersatz, so sinkt c.p. der Nettozinssatz der Föderation und umgekehrt. ${ }^{\prime}$

Diese Nettozinssatzänderung wird von einer Veränderung der Kapitallokation begleitet. Die Änderung der Kapitalmenge in Region a als Reaktion auf einen marginal veränderten Kapitalertragsteuersatz der Region a wird durch die partielle Ableitung der Kapitalmenge $\left(\mathrm{K}_{\mathrm{a}}\right)$ nach dem Steuersatz $\left(\mathrm{t}_{\mathrm{a}}\right)$ ausgedrückt: ${ }^{2}$

$$
\frac{\partial K_{a}}{\partial t_{a}}=\frac{-1}{\left(1-t_{a}\right)} \cdot \frac{-r}{\left(E\left(G_{a}\right) \cdot\left(1-t_{a}\right) \cdot f_{K a K d}\left(K_{a}\right)+E\left(G_{b}\right) \cdot\left(1-t_{b}\right) \cdot f_{K b K}\left(K_{b}\right)\right)} \text {. }
$$

Eine Erhöhung des Steuersatzes in Region a führt demnach unter sonst gleichen Umständen in dieser Region zu einem Kapitalabfluss und eine Senkung zu einem Kapitalzufluss: ${ }^{3}$

$$
\frac{\partial K_{a}}{\partial t_{a}}<0
$$

Da der Kapitalbestand der gesamten Föderation exogen vorgegeben ist, muss die von Region a abgeflossene Menge an Kapital Region b zufließen und umgekehrt. Insofern bewirkt eine Steuersatzänderung in Region a einen Kapitalzufluss bzw. -abfluss von Region b, der genau dem Kapitalabfluss bzw. -zufluss in Region a entspricht: ${ }^{4}$

$$
-\frac{\partial K_{a}}{\partial t_{a}}=\frac{\partial K_{b}}{\partial t_{a}} .
$$

Somit geht eine marginale Änderung des Kapitalertragsteuersatzes in Region a mit einer Änderung der Kapitalmenge in Region b vom Umfang

$$
\frac{\partial K_{b}}{\partial t_{a}}=\frac{-1}{\left(1-t_{a}\right)} \cdot \frac{r}{\left(E\left(G_{a}\right) \cdot\left(1-t_{a}\right) \cdot f_{K a K d}\left(K_{a}\right)+E\left(G_{b}\right) \cdot\left(1-t_{b}\right) \cdot f_{K b K d}\left(K_{b}\right)\right)}
$$

einher. ${ }^{5}$ Eine Erhöhung des Steuersatzes in Region a verursacht c.p. demnach, dass Kapital in Region b fließt und eine Senkung des Steuersatzes, dass Kapital aus Region $\mathrm{b}$ abflie $\beta \mathrm{t}:^{6}$

\footnotetext{
'Vgl. Hange / Wellisch (1998, S. 319).

${ }^{2}$ Die Berechnung, die zu Gleichung (3.27) führt, ist Anhang $4 \mathrm{~b}$ zu entnehmen.

${ }^{3}$ Vgl. Edwards / Keen (1996, S. 117), Homburg (1999, S. 3).

${ }^{4}$ Vgl. Apolte (2001, S. 368).

${ }^{5}$ Alternativ kann Gleichung (3.30) auch durch Ableitung von $\mathrm{K}_{\mathrm{b}}$ nach $\mathrm{t}_{\mathrm{a}}$ hergeleitet werden. Diese Herleitung ist in Anhang 4c aufgeführt.

${ }^{6}$ Vgl. Wildasin (1991, S. 398), Hange / Wellisch (1998, S. 319).
} 


$$
\frac{\partial K_{b}}{\partial t_{a}}>0
$$

Die Veränderungen des Nettozinssatzes und der Kapitallokation beruhen dabei auf folgendem Anpassungsprozess auf dem Kapitalmarkt: Zu Beginn ist der Kapitalmarkt im Gleichgewicht $\left(\mathrm{r}_{1}{ }^{*}, \mathrm{~K}_{\mathrm{i1}}{ }^{*}\right)$. Erhöht Region a beispielsweise einmalig den Kapitalertragsteuersatz, so sinkt bei konstanter Bruttorendite zunächst die Nettorendite dieser Region. Da es sich beim Kapitalertragsteuersatz um einen Lageparameter der Kapitalnachfragefunktion handelt, drückt sich dieser Vorgang grafisch in einer Verschiebung der Nachfragekurve von Region a nach links aus (siehe Abbildung 5). Weil die Nettorendite in Region a aufgrund der Steuererhöhung auf $\mathrm{r}_{\mathrm{a} 2}$ sinkt, liegt ein Ungleichgewicht auf dem Kapitalmarkt vor.

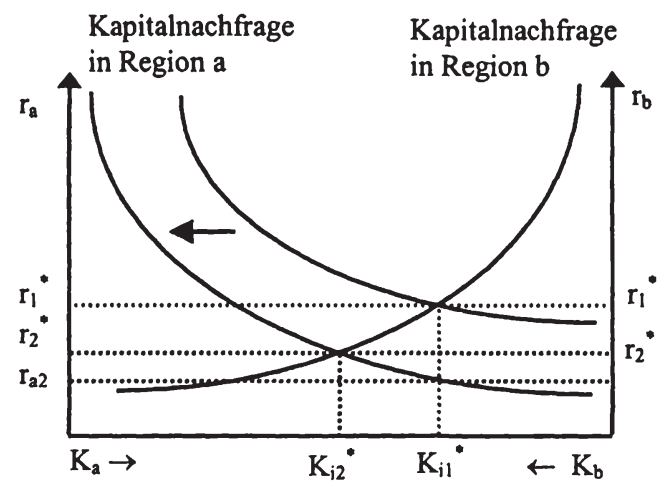

Abbildung 5: Wirkung einer Steuererhöhung in Region a

Die Kapitaleigner werden ihr Kapital von Region a abziehen und nach Region b verlagern, da sie dort eine höhere Nettorendite erzielen können. Dadurch steigt die Bruttorendite in Region a an, und die Bruttorendite in Region b fällt, was bei jetzt unveränderten Steuersätzen eine Änderung der Nettorenditen bewirkt. Nach entsprechender Wanderung des Kapitals von Region a in Region b, begleitet von einer Anpassung des Nettozinssatzes, erreicht der Kapitalmarkt sein neues Gleichgewicht $\left(\mathrm{r}_{2}{ }^{*}, \mathrm{~K}_{\mathrm{i} 2}{ }^{*}\right)$. 


\subsubsection{Wirkung des öffentlichen Zwischenprodukts}

Da das öffentliche Zwischenprodukt zu einem Anstieg in der Ausbringungsmenge führt, wird der Kapitalmarkt durch die Höhe der bereitgestellten Menge an öffentlichem Zwischenprodukt beeinflusst. Der Einfluss auf den Nettozinssatz wird mit Hilfe der Ableitung des Nettozinssatzes ( $r$ ) nach dem öffentlichen Zwischenprodukt $\left(\mathrm{G}_{\mathrm{a}}\right)$ ermittelt: ${ }^{1}$

$$
\frac{\partial r}{\partial G_{a}}=\frac{\frac{d E\left(G_{a}\right)}{d G_{a}}}{E\left(G_{a}\right)} \cdot \frac{r}{\left(1+\frac{E\left(G_{a}\right) \cdot\left(1-t_{a}\right) \cdot f_{K a K a}\left(K_{a}\right)}{E\left(G_{b}\right) \cdot\left(1-t_{b}\right) \cdot f_{K b K b}\left(K_{b}\right)}\right)} .
$$

Wie Gleichung (3.32) zu entnehmen ist, hängt der Nettozinssatz mit der Menge an bereitgestelltem öffentlichen Input positiv zusammen: ${ }^{2}$

$$
\frac{\partial r}{\partial G_{a}}>0 \text {. }
$$

Unter sonst gleichen Umständen erhöht (senkt) jede Regierung also den Nettozinssatz, indem sie mehr (weniger) öffentlichen Input anbietet.

Diese Zinssatzänderung geht mit einer Kapitalrelokation einher. Verändert beispielsweise Regierung a die Menge an öffentlichem Zwischenprodukt marginal, so führt dies zu einer Änderung der Kapitalmenge in eben dieser Region entsprechend Gleichung (3.34): ${ }^{3}$

$$
\frac{\partial K_{a}}{\partial G_{a}}=\frac{\frac{d E\left(G_{a}\right)}{d G_{a}}}{E\left(G_{a}\right)} \cdot\left(\frac{-r}{E\left(G_{a}\right) \cdot\left(1-t_{a}\right) \cdot f_{K a K d}\left(K_{a}\right)+E\left(G_{b}\right) \cdot\left(1-t_{b}\right) \cdot f_{K b K d}\left(K_{b}\right)}\right) .
$$

Wie zu erkennen ist, bewirkt c.p. eine Erhöhung der bereitgestellten Menge durch Regierung a einen Kapitalzufluss in Region a und eine Senkung einen Kapitalabfluss: ${ }^{4}$

$$
\frac{\partial K_{a}}{\partial G_{a}}>0
$$

\footnotetext{
'Für die Berechnung von Gleichung (3.32) siehe Anhang 5a.

${ }^{2}$ Vgl. Arnold / Fuest (1999, S. 167), Roloff (1999, S. 52; 2001, S. 118 f.).

${ }^{3} \mathrm{Vgl}$. für die Berechnung von Gleichung (3.34) Anhang 5b.

${ }^{4}$ Vgl. Keen / Marchand (1997, S. 37), Arnold / Fuest (1999, S. 167).
} 
Weil der Kapitalbestand der gesamten Föderation exogen vorgegeben und somit fix ist, entspricht der durch eine Erhöhung der bereitgestellten Menge an öffentlichem Zwischenprodukt induzierte Kapitalzufluss in Region a genau dem Kapitalabfluss aus Region $b$ und umgekehrt:

$$
-\frac{\partial K_{a}}{\partial G_{a}}=\frac{\partial K_{b}}{\partial G_{a}} \text {. }
$$

Insofern verursacht eine marginale Änderung der Bereitstellungsmenge an öffentlichem Input eine Änderung der Höhe des in Region b eingesetzten Kapitals von: ${ }^{1}$

$$
\frac{\partial K_{b}}{\partial G_{a}}=\frac{\frac{d E\left(G_{a}\right)}{d G_{a}}}{E\left(G_{a}\right)} \cdot \frac{r}{\left(E\left(G_{a}\right) \cdot\left(1-t_{a}\right) \cdot f_{K a K d}\left(K_{a}\right)+E\left(G_{b}\right) \cdot\left(1-t_{b}\right) \cdot f_{K b K d}\left(K_{b}\right)\right)} .
$$

Der Zusammenhang zwischen in Region a bereitgestelltem öffentlichen Zwischenprodukt und in Region $b$ attrahiertem Kapital ist somit negativ:

$$
\frac{\partial K_{b}}{\partial G_{a}}<0 \text {. }
$$

Der Veränderung des Nettozinssatzes und der Kapitallokation liegt dabei folgender Anpassungsprozess zugrunde. Zu Anfang befindet sich der Kapitalmarkt wiederum im Gleichgewicht $\left(\mathrm{r}_{1}{ }^{*}, \mathrm{~K}_{\mathrm{il}}{ }^{*}\right)$. Erhöht Region a nun beispielsweise die Menge an öffentlichem Zwischenprodukt, dann steigt die Bruttorendite dieser Region und somit bei konstantem Steuersatz auch die Nettorendite. Grafisch zeigt sich dieses in einer Verschiebung der Kapitalnachfragekurve nach rechts (siehe Abbildung 6). Da die Nettorendite in Region a $\left(r_{a 2}\right)$ in diesem Fall die Nettorendite der Region b übersteigt, besteht ein Ungleichgewicht auf dem Kapitalmarkt. Die Kapitaleigner werden ihr Kapital nun von Region b in Region a verlagern, weil sie dort eine höhere Nettorendite erwirtschaften können. Dadurch sinkt die Bruttorendite in Region a und die in Region b steigt, was bei jetzt unveränderten Steuersätzen zu einer Senkung der Nettorendite in Region a und zu einer Erhöhung der Nettorendite in Region b führt. Nach entsprechender Verlagerung des Kapitals und Anpassung des Nettozinssatzes wird schließlich der Gleichgewichtszustand $\left(\mathrm{r}_{2}{ }^{*}, \mathrm{~K}_{\mathrm{i} 2}{ }^{*}\right)$ erreicht.

\footnotetext{
${ }^{1}$ Diese Gleichung lässt sich auch durch Ableitung - wie in Anhang 5c gezeigt - ermitteln. 


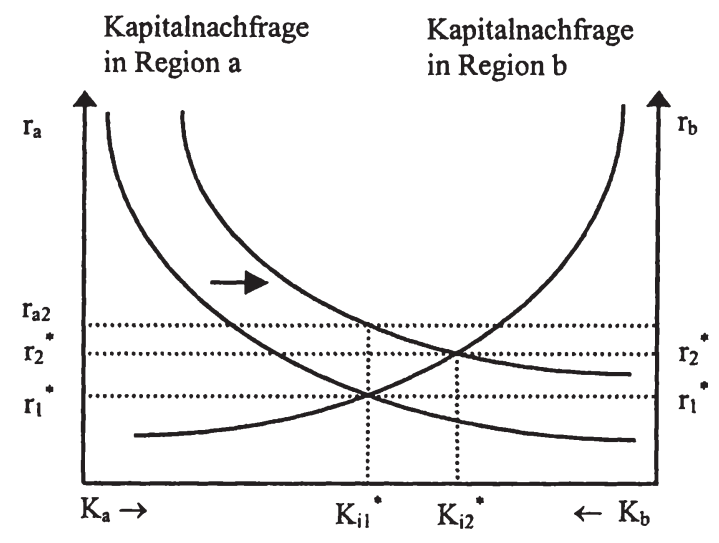

Abbildung 6: Wirkung einer Erhöhung der Bereitstellungsmenge des öffentlichen Zwischenprodukts in Region a

\subsection{Gesamtwirkung der fiskalpolitischen Instrumente einer Regierung}

Wie sich also gezeigt hat, haben die beiden fiskalpolitischen Instrumente eine vollkommen konträre Wirkung auf dem Kapitalmarkt. Bewirkt eine Senkung des Kapitalertragsteuersatzes eine Erhöhung des Nettozinssatzes, so bringt eine Senkung der Bereitstellungsmenge an öffentlichem Input ein Reduktion desselben und umgekehrt. Ebenso folgt auf eine Steuersatzerhöhung beispielsweise in Region a ein Abfluss des Kapitals aus Region a in Region b, wohingegen eine Erhöhung der Bereitstellungsmenge an öffentlichem Zwischenprodukt der Region a von einem Zufluss des Kapitals in Region a aus Region b begleitet wird. Diese Feststellung legt die Vermutung nahe, dass die beiden fiskalpolitischen Instrumente aus Sicht der Regierungen gegeneinander substituierbar sind. Die folgende Untersuchung wird wiederum beispielhaft nur für Region a durchgeführt. Die Ergebnisse gelten für Region b entsprechend. 


\subsubsection{Substituierbarkeit fiskalpolitischer Instrumente}

Um die Substituierbarkeit der fiskalpolitischen Instrumente zu bestimmen, bedarf es einer genaueren Untersuchung der gemeinsamen Wirkung von Steuern und öffentlichem Zwischenprodukt auf dem Kapitalmarkt: Zum einen wirken die fiskalpolitischen Instrumente auf die Kapitallokation. Somit ändert sich erstens die Höhe der von Region a attrahierten Kapitalmenge

$$
d K_{a}=\frac{\partial K_{a}}{\partial t_{a}} \cdot d t_{a}+\frac{\partial K_{a}}{\partial G_{a}} \cdot d G_{a}
$$

und zweitens ändert sich die Höhe der in Region b eingesetzten Kapitalmenge

$$
d K_{b}=\frac{\partial K_{b}}{\partial t_{a}} \cdot d t_{a}+\frac{\partial K_{b}}{\partial G_{a}} \cdot d G_{a}
$$

bei einer marginalen Änderung der fiskalpolitischen Instrumente. Zum anderen beeinflussen sie aber auch die Höhe des Nettozinssatzes wie folgt:

$$
d r=\frac{\partial r}{\partial t_{a}} \cdot d t_{a}+\frac{\partial r}{\partial G_{a}} \cdot d G_{a} .
$$

Um nun die Substituierbarkeit der beiden Instrumente zu ermitteln, wird verlangt, dass die Änderung der fiskalpolitischen Instrumente zu keinerlei Änderung auf dem Kapitalmarkt führt. Diese Bedingung ist erfüllt, wenn sich sowohl die Kapitallokation als auch der Nettozinssatz nicht ändern:

$$
d K_{a}=d K_{b}=d r=0 .
$$

Unter Berücksichtigung von Gleichung (3.25), Gleichung (3.27), Gleichung (3.30), Gleichung (3.32), Gleichung (3.34) und Gleichung (3.37) zeigt sich, dass eine Änderung der fiskalpolitischen Instrumente keine Änderung auf dem Kapitalmarkt bewirkt, wenn die Änderung derart vorgenommen wird, dass

$$
0=\frac{-1}{\left(1-t_{a}\right)} \cdot d t_{a}+\frac{\frac{d E\left(G_{a}\right)}{d G_{a}}}{E\left(G_{a}\right)} \cdot d G_{a}
$$

gilt.' Welche Beträge der fiskalpolitischen Instrumente sich gegeneinander substituieren lassen, kann ermittelt werden, indem Gleichung (3.43) integriert wird. Dadurch erhält man folgende Stammfunktion:

\footnotetext{
${ }^{1}$ Vgl. Anhang 6.
} 
(3.44)

$$
0=\ln \left(\left(1-t_{a}\right) \cdot E\left(G_{a}\right)\right)-c,
$$

wobei c einen beliebigen Wert repräsentiert. ${ }^{1}$ Durch Umformung gelangt man zur Substitutionsfunktion, die den Steuersatz in Abhängigkeit der Menge an öffentlichem Input darstellt:

$$
t_{a}=1-\frac{e^{c}}{E\left(G_{a}\right)} .
$$

Sie gibt alle möglichen Kombinationen von Steuersatz und öffentlichem Zwischenprodukt wieder, durch die eine identische Kapitallokation und ein identischer Nettozinssatz erreicht wird.

Die Substitutionsfunktion hat eine positive Steigung

$$
\frac{\partial t_{a}}{\partial G_{a}}=\frac{e^{c} \cdot \frac{d E\left(G_{a}\right)}{d G_{a}}}{\left[E\left(G_{a}\right)\right]^{2}}>0,
$$

weshalb eine Erhöhung des Steuersatzes durch eine entsprechende Erhöhung der Bereitstellungsmenge an öffentlichem Input kompensiert werden kann und umgekehrt.

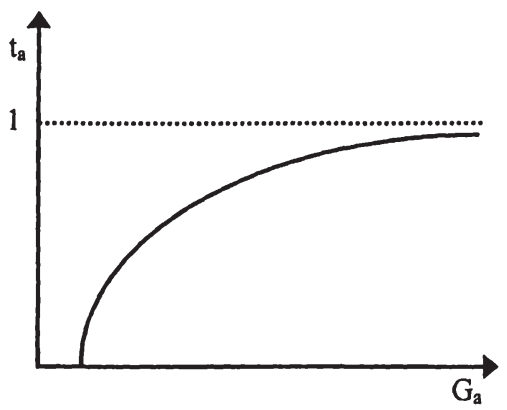

Abbildung 7: Substitutionsfunktion

Aus der zweiten Ableitung ist ersichtlich, dass ihr Verlauf rechts gekrümmt ist:

\footnotetext{
${ }^{1}$ Eine ökonomische Interpretation des Wertes c wird in Abschnitt 3.3.2 ff. geliefert. Dass es sich bei Gleichung (3.44) um die Stammfunktion von Gleichung (3.43) handelt, kann durch Bilden des Totalen Differentials von Gleichung (3.44) überprüft werden.
} 


$$
\frac{\partial^{2} t_{a}}{\partial G_{a}^{2}}=\frac{e^{c} \cdot \frac{d^{2} E\left(G_{a}\right)}{d G_{a}^{2}}}{\left[E\left(G_{a}\right)\right]^{2}}-\frac{2 \cdot e^{c} \cdot\left[\frac{d E\left(G_{a}\right)}{d G_{a}}\right]^{2}}{\left[E\left(G_{a}\right)\right]^{3}}<0 .
$$

Die Substitutionsfunktion ist in Abbildung 7 grafisch dargestellt. Aus ihr kann entnommen werden, dass, wenn der Ausgangssteuersatz nahe dem Wert 1 liegt, es zur Kompensation einer Steuersatzänderung nahezu einer unendlich großen Änderung der Menge an öffentlichem Zwischenprodukt bedarf. Befindet sich der Ausgangssteuersatz hingegen nahe dem Wert 0, so wird zur Kompensation einer Steuersatzänderung nur eine kleine Änderung in der Menge an öffentlichem Input benötigt.

\subsubsection{Fiskalpolitischer Instrumenten-Index}

Betrachtet man Gleichung (3.45), so zeigt sich, dass die Substitutionsfunktion maßgeblich von dem Wert $\mathrm{c}$ bestimmt wird. Je nach dem wie hoch der Wert ausfällt, erhält man eine andere Substitutionsfunktion. In Abbildung 8 sind Substitutionsfunktionen mit unterschiedlichen Werten von $\mathrm{c}$ abgetragen.

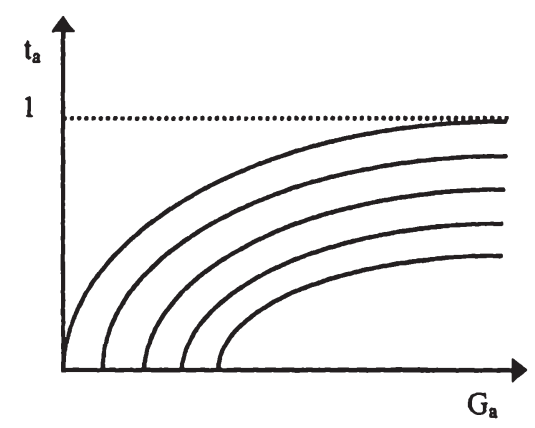

Abbildung 8: Kurvenschar der Substitutionsfunktionen

Je höher der Wert c ist, desto weiter unten bzw. rechts befindet sich die Substitutionskurve. Es handelt sich bei diesem Wert also um einen Lageparameter der Substitutionsfunktion. Er beschreibt das Niveau beider fiskalpolitischer Mittel zusammengefasst: 


$$
c=\ln \left(\left(1-t_{a}\right) \cdot E\left(G_{a}\right)\right)
$$

(siehe Gleichung (3.44)). Aufgrund dieser Eigenschaft wird er im weiteren als fiskalpolitischer Instrumenten-Index (I) oder einfach nur als Instrumenten-Index bezeichnet. Insofern wird der Instrumenten-Index der Region a $\left(I_{a}\right)$ definiert als:

$$
I_{a}=\ln \left(\left(1-t_{a}\right) \cdot E\left(G_{a}\right)\right) \text {. }
$$

Werden nun der Kapitalertragsteuersatz und die Bereitstellungsmenge des öffentlichen Inputs derart geändert, dass sich die Wirkungen der Änderungen gegenseitig aufheben (siehe Bedingung (3.43)), so drückt sich dieses in einem konstanten Instrumenten-Index aus. Ein konstanter Index weißt also darauf hin, dass keine Veränderungen auf dem Kapitalmarkt ausgelöst werden. Grafisch zeigt sich eine solche Änderung der fiskalpolitischen Instrumente in einer Bewegung auf einer Substitutionskurve. Eine Veränderung der Situation auf dem Kapitalmarkt kann also nur durch Änderungen des Steuersatzes und der Menge an öffentlichem Zwischenprodukt erfolgen, die zu einer Änderung des fiskalpolitischen Instrumenten-Indexes führen. Nur dann bewirken nämlich die Änderungen, dass die Substitutionskurve verlassen wird. Daher wird im folgenden untersucht, welche Änderungen der fiskalpolitischen Instrumente mit welcher Art von Änderung des fiskalpolitischen Instrumenten-Indexes einhergehen. Dabei wird zunächst die Wirkung eines einzelnen fiskalpolitischen Instruments Steuern oder öffentliches Zwischenprodukt - auf den Index betrachtet. AnschlieBend wird dann die Wirkung eines kombinierten Einsatzes der Instrumente analysiert. Die Frage, wie sich eine Änderung des Instrumenten-Indexes auf den Kapitalmarkt bezüglich der attrahierten Kapitalmengen und des Nettozinssatzes auswirkt, wird zunächst zurückgestellt und erst in Abschnitt 3.4 untersucht.

\subsubsection{Wirkung eines fiskalpolitischen Instruments auf den Instrumenten-Index}

Welche Wirkung eine Senkung bzw. Erhöhung des Steuersatzes auf die Höhe des Instrumenten-Indexes hat, hängt von der damit einhergehenden Änderung der Bereitstellungsmenge an öffentlichem Input ab. Führt die Regierung einzig und allein eine Steuererhöhung (Steuersenkung) durch und lässt die Bereitstellungsmenge unverändert, so bewirkt diese eine Senkung (Erhöhung) ihres Indexes, da 
$(3.50)$

$$
\frac{\partial I_{a}}{\partial t_{a}}=\frac{-1}{\left(1-t_{a}\right)}<0
$$

gilt. Der Zusammenhang zwischen Steuersatz und Instrumenten-Index einer Region ist demnach negativ. Diese Aussage ist in Abbildung 9 für Region a verdeutlicht:

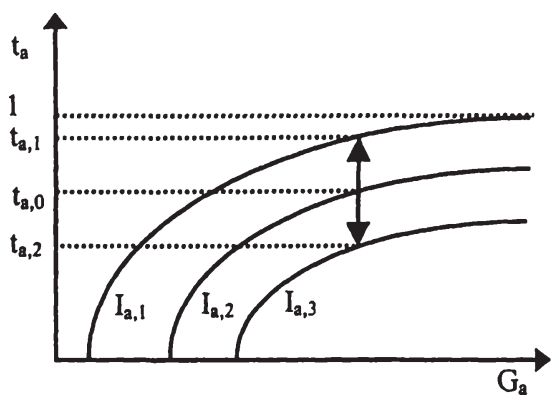

Abbildung 9: Wirkung einer Änderung des Steuersatzes auf den InstrumentenIndex

Wird der Steuersatz von $t_{a, 0}$ auf $t_{a, 1}$ erhöht und bleibt die Bereitstellungsmenge an öffentlichem Input konstant, dann wird das Niveau $I_{a, 2}$ verlassen und das niedrigere Niveau $I_{a, 1}$ erreicht. Wird der Steuersatz hingegen von $t_{a, 0}$ auf $t_{a, 2}$ gesenkt, so gelangt man zu dem höheren Niveau $I_{a, 3}$.

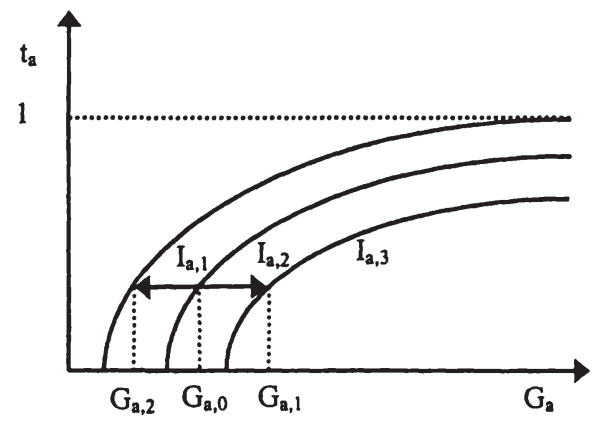

Abbildung 10: Wirkung der Bereitstellungsmenge an öffentlichem Zwischenprodukt auf den Instrumenten-Index 
Wird einzig und allein die Menge an öffentlichem Zwischenprodukt verändert, so geht diese Änderung mit einer gleichgerichteten Änderung des fiskalpolitischen Instrumenten-Indexes einher. Der Zusammenhang zwischen öffentlichem Zwischenprodukt und Instrumenten-Index ist also positiv, da

$$
\frac{\partial I_{a}}{\partial G_{a}}=\frac{\frac{d E\left(G_{a}\right)}{d G_{a}}}{E\left(G_{a}\right)}>0
$$

vorliegt. Dies ist in Abbildung 10 beispielhaft für Region a veranschaulicht. Wird die Menge an öffentlichem Input von $G_{a, 0}$ auf $G_{a, 1}$ erhöht und bleibt der Kapitalertragsteuersatz konstant, dann wird ein höheres Niveau $\left(I_{a, 3}\right)$ des fiskalpolitischen Instrumenten-Indexes erreicht. Wird hingegen die Bereitstellungsmenge von $\mathrm{G}_{\mathrm{a}, 0}$ auf $\mathrm{G}_{\mathrm{a}, 2}$ gesenkt, dann verringert sich der Index, so dass auf das Niveau $I_{a, 1}$ zurückgefallen wird.

\subsubsection{Gesamtwirkung der fiskalpolitischen Instrumente auf den Instrumenten-} Index

Ändert eine Regierung nun gleichzeitig die Höhe des Steuersatzes und die Menge an öffentlichem Input, so ist das Ergebnis nicht mehr ganz so eindeutig. Wie in Abschnitt 3.3.1 gezeigt, kompensieren sich unter Umständen die Effekte der beiden fiskalpolitischen Instrumente. Allgemein lässt sich die Änderung des Indexes bestimmen, indem das Totale Differential der Gleichung (3.49) gebildet wird:

$$
d I_{a}=\frac{-1}{\left(1-t_{a}\right)} \cdot d t_{a}+\frac{\frac{d E\left(G_{a}\right)}{d G_{a}}}{E\left(G_{a}\right)} \cdot d G_{a} .
$$

Ein höheres Niveau des fiskalpolitischen Instrumenten-Indexes wird also immer dann erreicht, wenn

$$
\frac{-1}{\left(1-t_{a}\right)} \cdot d t_{a}+\frac{\frac{d E\left(G_{a}\right)}{d G_{a}}}{E\left(G_{a}\right)} \cdot d G_{a}>0
$$

erfüllt ist und ein niedrigeres, wenn 


$$
\frac{-1}{\left(1-t_{a}\right)} \cdot d t_{a}+\frac{\frac{d E\left(G_{a}\right)}{d G_{a}}}{E\left(G_{a}\right)} \cdot d G_{a}<0
$$

gilt.

Senkt die Regierung nun ihre Steuern $\left(\mathrm{dt}_{\mathrm{a}}<0\right)$, so wird ein höheres Niveau des Instrumenten-Indexes nur erzielt, falls das Verhältnis der marginalen Änderungen der beiden fiskalpolitischen Instrumente kleiner ist als das Verhältnis vom Effizienzparameter zu dem Produkt aus Steuerfaktor und marginaler Effizienzsteigerung:

$$
\frac{d G_{a}}{d t_{a}}<\frac{E\left(G_{a}\right)}{\left(1-t_{a}\right) \cdot \frac{d E\left(G_{a}\right)}{d G_{a}}} .
$$

Bedingung (3.55) ist stets gegeben, sollte die Steuersatzsenkung von einer Erhöhung der Menge an öffentlichem Zwischenprodukt begleitet werden. Geht jedoch die Steuersatzsenkung mit einer Senkung der Bereitstellungsmenge an öffentlichem Input einher, um die Steuersenkung durch eine Ausgabensenkung gegen zu finanzieren, so hängt das Ausmaß einer möglichen Senkung der Bereitstellungsmenge vom Ausgangsniveau des Steuersatzes ab.

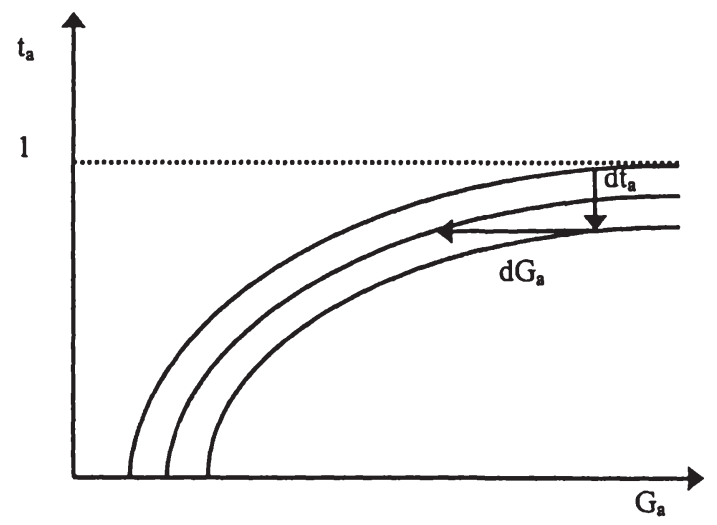

Abbildung 11: Wirkung einer „Senkungspolitik“ in einer Hochsteuerregion

Handelt es sich um eine Hochsteuerregion mit einem sehr hohen Kapitalertragsteuersatz, dann trifft Bedingung (3.55) auch dann noch zu, wenn eine relativ geringe Steuersatzsenkung mit einer relativ hohen Senkung der Menge an 
öffentlichem Zwischenprodukt kombiniert wird (vgl. Abbildung 11) und gilt somit zwangsläufig ebenfalls, wenn die Senkung der beiden fiskalpolitischen Instrumente in etwa proportional ausfällt. Für den Fall einer Hochsteuerregion ist also davon auszugehen, dass eine durch Verringerung der Ausgaben für öffentliche Zwischenprodukte finanzierte Steuersenkung zu einem höheren fiskalpolitischen Instrumenten-Index führt.

Liegt jedoch eine Region vor, die sich ohnehin eher durch einen niedrigen Kapitalertragsteuersatz auszeichnet, so kann Bedingung (3.55) nur erfüllt werden, wenn neben einer großen Steuersatzsenkung allenfalls eine sehr geringe Reduktion der Menge an öffentlichem Zwischenprodukt erfolgt (siehe Abbildung 12).

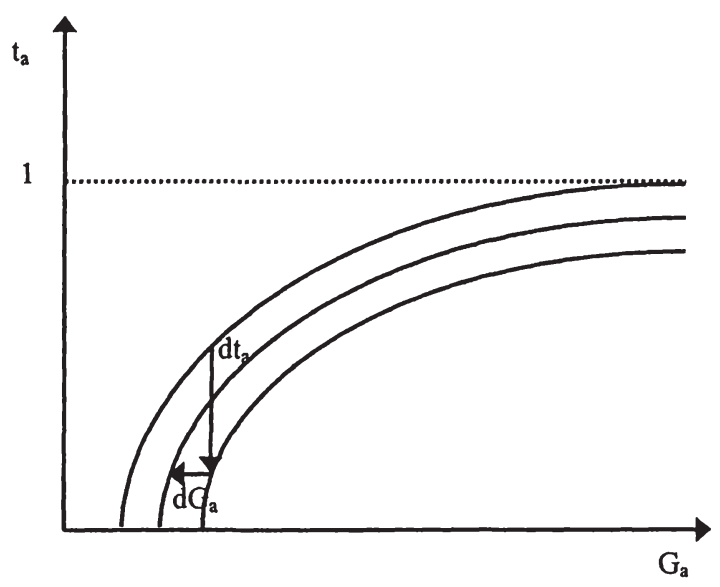

Abbildung 12: „Senkungspolitik“ bei defizitärem Budget in einer Niedrigsteuerregion

Dann ist jedoch die Wahrscheinlichkeit sehr hoch, dass die geringe Ausgabenverminderung nicht ausreicht, um die Steuersatzsenkung gegen zu finanzieren. Betreibt eine Niedrigsteuerregion eine Senkung der Steuern und Ausgaben und möchte sie dabei ein ausgeglichenes Budget beibehalten, so ist davon auszugehen, dass diese durch ihre Politik ihren fiskalpolitischen Instrumenten-Index reduziert. Erfolgt nämlich eine etwa proportionale Änderung der beiden fiskalpolitischen Instrumente, dann wird in einer Niedrigsteuerregion der positive Effekt 
der Steuersatzsenkung durch den negativen Effekt der Ausgabenreduktion überkompensiert (siehe Abbildung 13).

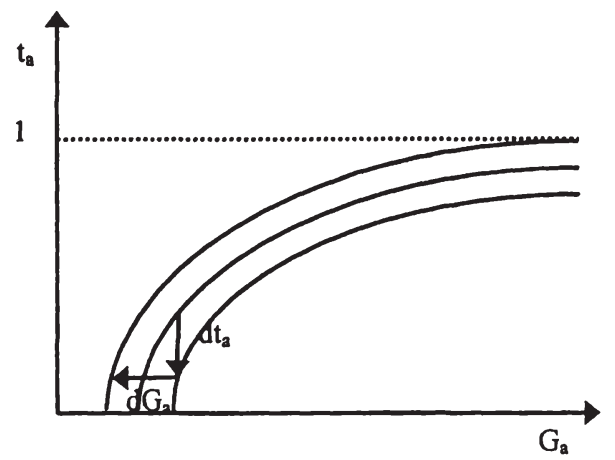

Abbildung 13: „Senkungspolitik“ bei ausgeglichenem Budget in einer Niedrigsteuerregion

Senkt die Regierung ihren Steuersatz nun nicht, sondern erhöht sie diesen, so kann ein höherer fiskalpolitischer Instrumenten-Index nur erreicht werden, wenn

$$
\frac{d G_{a}}{d t_{a}}>\frac{E\left(G_{a}\right)}{\left(1-t_{a}\right) \cdot \frac{d E\left(G_{a}\right)}{d G_{a}}}
$$

vorliegt (vgl. Gleichung (3.53)). Diese Bedingung kann nicht zutreffen, wenn die Steuersatzerhöhung mit einer Senkung der Bereitstellungsmenge an öffentlichem Input einher geht. Wird eine Steuersatzerhöhung von einer Senkung der Menge an öffentlichem Zwischenprodukt begleitet, so wird dadurch stets der fiskalpolitische Instrumenten-Index verringert. Eine Steuersatzerhöhung muss demnach immer mit einem entsprechend starken Anstieg der Bereitstellungsmenge an öffentlichem Input verbunden sein, um den Index zu erhöhen. Inwiefern die Steuersatzerhöhung gekoppelt mit einem Anstieg der Menge an öffentlichem Input zu einer Erhöhung des Instrumenten-Indexes führt, hängt wiederum vom Ausgangsniveau des Steuersatzes ab. Je geringer das Ausgangsniveau des Steuersatzes ist, desto geringer kann die Erhöhung der Bereitstellungsmenge bei einer Erhöhung des Steuersatzes ausfallen, um dennoch ein höheres Niveau des fiskalpolitischen Instrumenten-Indexes zu erreichen. 
In einer Hochsteuerregion muss demnach die Steuersatzerhöhung von einem starken Anstieg der Menge an öffentlichem Input begleitet werden (vgl. Abbildung 14), so dass diese Maßnahme wohl eher nicht Budget-neutral durchzuführen ist.

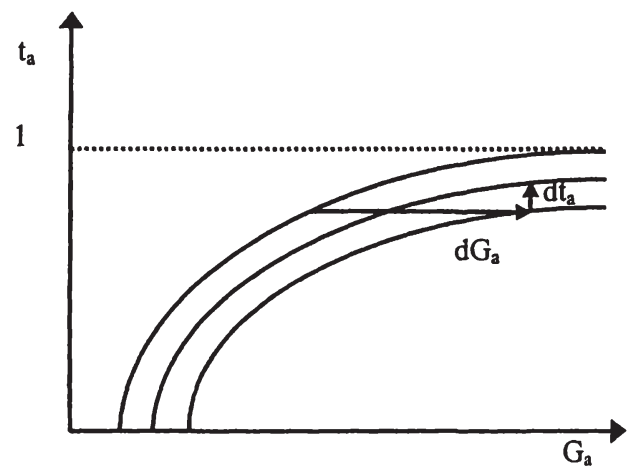

Abbildung 14: „Erhöhungspolitik“ bei defizitärem Budget in einer Hochsteuerregion

Wird hingegen eine Erhöhung beider fiskalpolitischer Instrumente bei ausgeglichenem Budget gefordert, dann ist es sehr wahrscheinlich, dass der fiskalpolitische Instrumenten-Index durch diese Vorgehensweise reduziert wird (siehe Abbildung 15).

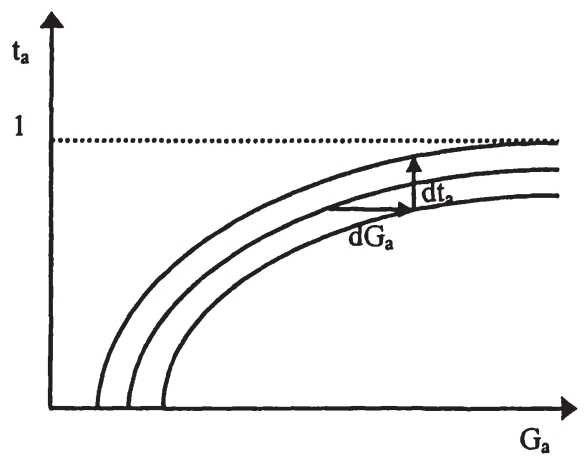

Abbildung 15: „Erhöhungspolitik“ bei ausgeglichenem Budget in einer Hochsteuerregion 
Handelt es sich hingegen um eine Niedrigsteuerregion, so kann die Steuersatzerhöhung mit einer relativ niedrigen Erhöhung der Ausgaben einhergehen, so dass ein höheres Niveau des Instrumenten-Indexes erreicht wird (vgl. Abbildung 16). Die jeweilige Regierung kann somit die Ausgaben erhöhen, um das Niveau des fiskalpolitischen Instrumenten-Indexes anzuheben, und die Ausgabenerhöhung durch entsprechende Steuererhöhungen finanzieren.

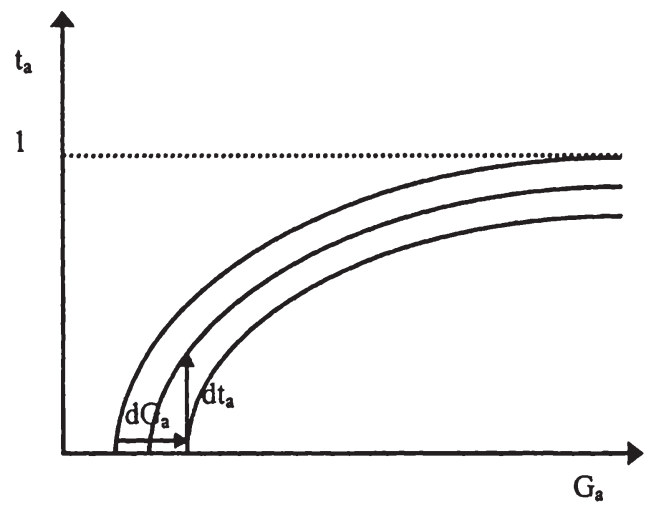

Abbildung 16: Wirkung einer „Erhöhungspolitik“ in einer Niedrigsteuerregion

Zusammenfassend kann also festgehalten werden, dass es von dem Ausgangsniveau des Kapitalertragsteuersatzes in einer Region abhängt, welche Maßnahmen einer Regierung zu einer Erhöhung bzw. Senkung des fiskalpolitischen Instrumenten-Indexes führen. Liegt eine Region vor, die durch einen hohen Steuersatz gekennzeichnet ist, so lässt sich ein höheres Niveau des Instrumenten-Indexes am leichtesten erzielen, indem der Steuersatz gesenkt wird. Die Steuersatzsenkung führt selbst dann zu einem höheren Index, wenn sie durch eine entsprechende Senkung der Menge an öffentlichem Zwischenprodukt finanziert wird. Wird hingegen der Steuersatz noch weiter erhöht, so kann wahrscheinlich selbst durch eine mit Hilfe der Steuererhöhung finanzierte Erhöhung der Menge an öffentlichem Zwischenprodukt das Abrutschen auf ein niedrigeres Niveau nicht verhindert werden. Handelt es sich hingegen um eine Region, dessen Kapitalertragsteuersatz sich ohnehin schon auf einem geringen Niveau befindet, so kann ein höheres Niveau des Instrumenten-Indexes am besten erreicht werden, wenn die Menge an öffentlichem Zwischenprodukt erhöht wird. Sogar bei einer 
gleichzeitigen Erhöhung des Steuersatzes zur Finanzierung der so entstandenen Mehrausgaben steigt der fiskalpolitische Instrumenten-Index in dem Fall an. Wird allerdings die Menge an öffentlichem Input zurückgefahren, dann wird aller Wahrscheinlichkeit nach selbst bei einer gleichzeitig vorgenommenen Steuersatzsenkung der Instrumenten-Index verringert.

\subsection{Wettbewerb um Kapital}

Die Regierungen der einzelnen Regionen setzen nun ihre fiskalpolitischen Instrumente ein, um Kapital zu attrahieren. Allerdings bestimmen nicht die einzelnen Regierungen für sich genommen die Höhe der in ihrer Region eingesetzten Kapitalmenge. Die attrahierte Kapitalmenge einer Region wird auch von den Aktionen der Konkurrenzregion beeinflusst. Insofern führen Änderungen des fiskalpolitischen Instrumenten-Indexes sowohl von Region a als auch von Region $\mathrm{b}$ zu einer Kapitalrelokation

$$
\begin{aligned}
& d K_{a}=\frac{\partial K_{a}}{\partial I_{a}} \cdot d I_{a}+\frac{\partial K_{a}}{\partial I_{b}} \cdot d I_{b}, \\
& d K_{b}=\frac{\partial K_{b}}{\partial I_{a}} \cdot d I_{a}+\frac{\partial K_{b}}{\partial I_{b}} \cdot d I_{b}
\end{aligned}
$$

und zu einer Änderung des Nettozinssatzes

$$
d r=\frac{\partial r}{\partial I_{a}} \cdot d I_{a}+\frac{\partial r}{\partial I_{b}} \cdot d I_{b}
$$

Um also die Wirkung der von den Regierungen vorgenommenen Fiskalpolitik zu ermitteln, muss zuerst die Wirkung des fiskalpolitischen InstrumentenIndexes auf die Kapitalmenge in Region a und Region $b$ und auf den Nettozinssatz bestimmt werden.

\subsubsection{Wirkung des fiskalpolitischen Instrumenten-Indexes}

Ein Gleichgewicht auf dem Kapitalmarkt liegt nach Gleichung (3.24) vor, wenn folgende Bedingung erfüllt ist: 


$$
\bar{K}=f_{\mathrm{P} a}\left(\frac{r}{E\left(G_{a}\right) \cdot\left(1-t_{a}\right)}\right)+f_{\mathrm{Pb}}\left(\frac{r}{E\left(G_{b}\right) \cdot\left(1-t_{b}\right)}\right) .
$$

Berücksichtigt man, dass nach Gleichung (3.49)

$$
e^{I_{i}}=\left(1-t_{i}\right) \cdot E\left(G_{i}\right)
$$

gilt, so kann die Gleichgewichtsbedingung des Kapitalmarkts auch in Abhängigkeit der fiskalpolitischen Instrumenten-Indizes der Regionen $\mathrm{a}$ und $\mathrm{b}$ ausgedrückt werden:

$$
\bar{K}=f_{\mathrm{Pa}}\left(\frac{r}{e^{I_{a}}}\right)+f_{\mathrm{Pb}}\left(\frac{r}{e^{I_{b}}}\right) .
$$

Aus Bedingung (3.62) erhält man für die Wirkung des Instrumenten-Indexes der Region a auf den Nettozinssatz: ${ }^{1}$

$$
\frac{\partial r}{\partial I_{a}}=\frac{r}{\left(1+\frac{e^{I_{a}} \cdot f_{K a K a}\left(K_{a}\right)}{e^{I_{b}} \cdot f_{K b K b}\left(K_{b}\right)}\right)} .
$$

Eine Erhöhung des fiskalpolitischen Instrumenten-Indexes der Region a führt also c.p. zu einer Erhöhung des Nettozinssatzes und umgekehrt:

$$
\frac{\partial r}{\partial I_{a}}>0
$$

Entsprechendes gilt für eine Änderung des Instrumenten-Indexes von Region b:

$$
\frac{\partial r}{\partial I_{b}}=\frac{r}{\left(1+\frac{e^{I_{b}} \cdot f_{K b K b}\left(K_{b}\right)}{e^{I_{a}} \cdot f_{K a K a}\left(K_{a}\right)}\right)}>0 \text {. }
$$

Diese Zinssatzänderung wird von einer Kapitalrelokation begleitet. Verändert Region a zum Beispiel die Höhe ihres fiskalpolitischen Instrumenten-Indexes marginal, dann bewirkt dieses eine Änderung der von Region a attrahierten Kapitalmenge wie folgt: ${ }^{2}$

$$
\frac{\partial K_{a}}{\partial I_{a}}=\frac{-r}{\left(e^{I_{a}} \cdot f_{K a K a}\left(K_{a}\right)+e^{I_{b}} \cdot f_{K b K b}\left(K_{b}\right)\right)} .
$$

Entsprechend ergibt sich für Region $b$ :

${ }_{2}^{1}$ Die Berechnung von Gleichung (3.63) ist Anhang 7a zu entnehmen.

${ }^{2}$ Die Berechnung fur Gleichung (3.66) ist im Anhang 7b enthalten. 


$$
\frac{\partial K_{b}}{\partial I_{b}}=\frac{-r}{\left(e^{I_{a}} \cdot f_{K a K a}\left(K_{a}\right)+e^{I_{b}} \cdot f_{K b K b}\left(K_{b}\right)\right)} .
$$

Die Wirkung des fiskalpolitischen Instrumenten-Indexes einer Region auf die in dieser Region attrahierte Kapitalmenge ist demnach für beide Regionen gleich, da nach Gleichung (3.66) und Gleichung (3.67)

$$
\frac{\partial K_{a}}{\partial I_{a}}=\frac{\partial K_{b}}{\partial I_{b}}
$$

gilt. Wie aus diesen Gleichungen darüber hinaus zu entnehmen ist, hängen die attrahierte Kapitalmenge einer Region und der fiskalpolitische InstrumentenIndex derselben Region positiv zusammen:

$$
\frac{\partial K_{a}}{\partial I_{a}}=\frac{\partial K_{b}}{\partial I_{b}}>0
$$

Unter sonst gleichen Umständen führt also eine Erhöhung des InstrumentenIndexes einer Region zu einer höheren Kapitalmenge in eben dieser Region und umgekehrt. Da der Kapitalbestand der Föderation exogen vorgegeben und fix ist, entspricht der Kapitalzufluss (Kapitalabfluss) einer Region dem Kapitalabfluss (Kapitalzufluss) der Konkurrenzregion, weshalb

$$
\frac{\partial K_{a}}{\partial I_{a}}=-\frac{\partial K_{b}}{\partial I_{a}}
$$

bzw.

$$
\frac{\partial K_{b}}{\partial I_{b}}=-\frac{\partial K_{a}}{\partial I_{b}}
$$

vorliegt. ${ }^{1}$ Insofern reduziert c.p. eine Erhöhung des fiskalpolitischen Instrumenten-Indexes einer Region die von der Konkurrenzregion attrahierte Kapitalmenge und umgekehrt:

$$
\frac{\partial K_{a}}{\partial I_{b}}=\frac{\partial K_{b}}{\partial I_{a}}<0
$$

Die Kapitalrelokation und die Änderung des Nettozinssatzes beruhen dabei auf folgendem Anpassungsprozess des Kapitalmarkts: Im Ausgangszustand ( $\mathrm{r}_{1}{ }^{*}$, $\mathrm{K}_{\mathrm{il}}{ }^{\text {) }}$ ) befindet sich der Kapitalmarkt im Gleichgewicht. Wird nun beispielsweise

${ }^{1}$ Für die Berechnung von $\frac{\partial K_{b}}{\partial I_{a}}$ siehe Anhang 7c. 
von Region a der fiskalpolitische Instrumenten-Index auf welche Weise auch immer angehoben, so verschiebt sich die Nachfragekurve dieser Region nach rechts (vgl. Abbildung 17).

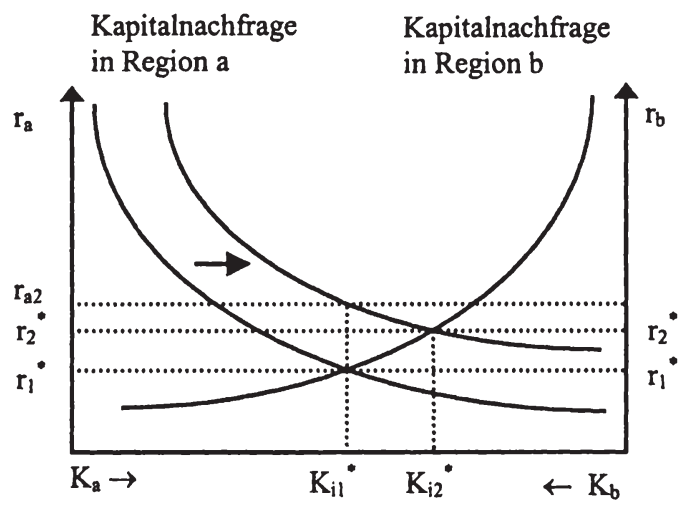

Abbildung 17: Wirkung einer Erhöhung des fiskalpolitischen InstrumentenIndexes von Region a

Es liegt nun ein Ungleichgewicht auf dem Kapitalmarkt vor, weil die Nettorendite in Region a $\left(\mathrm{r}_{\mathrm{a} 2}\right)$ höher ausfällt als die Nettorendite der Region b. Die Kapitaleigner werden daraufhin ihr Kapital von Region b in Region a verlagern, da sie dort eine höhere Nettorendite erzielen können. Dadurch steigt die Bruttorendite in Region b, wobei die in Region a sinkt, was bei jetzt unveränderten Steuersätzen eine entsprechende Änderung der Nettorenditen mit sich bringt. Durch die Verlagerung des Kapitals und die Anpassung des Nettozinssatzes gelangt man schließlich zum Gleichgewichtszustand $\left(\mathrm{r}_{2}{ }^{*}, \mathrm{~K}_{\mathrm{i} 2}{ }^{*}\right)$.

\subsubsection{Wettbewerb der Regionen}

An dieser Stelle können nun endlich die Bedingungen heraus gearbeitet werden, unter denen Region a bzw. b zusätzliches Kapital attrahiert. Nach Gleichung (3.68), Gleichung (3.70) und Gleichung (3.71) gilt: 


$$
\frac{\partial K_{a}}{\partial I_{a}}=\frac{\partial K_{b}}{\partial I_{b}}=-\frac{\partial K_{b}}{\partial I_{a}}=-\frac{\partial K_{a}}{\partial I_{b}} .
$$

Insofern zieht Region a zusätzliches Kapital an, wenn

$$
d K_{a}=\frac{\partial K_{a}}{\partial I_{a}} \cdot\left(d I_{a}-d I_{b}\right)>0
$$

bzw. Region b wenn

$$
d K_{b}=\frac{\partial K_{a}}{\partial I_{a}} \cdot\left(d I_{b}-d I_{a}\right)>0
$$

vorliegt (vgl. Gleichung (3.57) und Gleichung (3.58)). Inwiefern eine Region zusätzlich Kapital anlocken kann, hängt also von der Differenz der Änderungen der fiskalpolitischen Instrumenten-Indizes beider Regionen ab. Senkt die Konkurrenzregion ihren Index, so kann bereits durch eine etwas geringer ausfallende Senkung des Indexes zusätzliches Kapital von der Region angezogen werden. Erhöht hingegen die Konkurrenzregion ihren Index, so muss die andere Region diese Erhöhung überbieten, um noch weiteres Kapital attrahieren zu können. Fällt die Erhöhung des Instrumenten-Indexes einer Region geringer aus als die Erhöhung des Indexes der Konkurrenzregion, dann hat die Region sogar mit Kapitalabflüssen zu rechnen.

Darüber hinaus hat eine Regierung, die entsprechende fiskalpolitische Maßnahmen ergreift, zu beachten, dass ihre Aktionen auf die Höhe des Nettozinssatzes wirken. Eine Nettozinssatzänderung wird nach Gleichung (3.59) u.a. von einer Änderung der beiden Indizes ausgelöst:

$$
d r=\frac{\partial r}{\partial I_{a}} \cdot d I_{a}+\frac{\partial r}{\partial I_{b}} \cdot d I_{b} .
$$

Aus Gleichung (3.63) bis Gleichung (3.65) folgt, dass der Nettozinssatz bei einer Erhöhung der Indizes in beiden Regionen steigt und bei einer Senkung der Indizes sinkt. Erhöht die eine Region ihren Index und senkt die andere ihren, so wird vom Umfang der Änderungen der Indizes, von den Ausgangswerten der Indizes sowie des Nettozinssatzes und von der Steigung der Grenzproduktivitäten des Kapitals in beiden Regionen bestimmt, wie sich der Nettozinssatz ändert. 


\subsubsection{Ruinöse Wettbewerbsspirale}

Es liege nun eine Situation vor, in der ein Gleichgewicht auf dem Kapitalmarkt besteht. Beide Regierungen verfolgen gleichzeitig das Ziel, dauerhaft zusätzliches Kapital zu attrahieren. Daher erhöhen sowohl Region a als auch Region b ihren Instrumenten-Index. Der Einfachheit halber sei unterstellt, dass die Erhöhung in beiden Regionen gleich groß ausfällt, so dass

$$
d I_{a}=d I_{b}>0
$$

gilt. Insofern hebt sich die Wirkung der Änderungen der fiskalpolitischen Instrumente der beiden konkurrierenden Regionen bezüglich der Kapitallokation auf (vgl. Gleichung (3.74) und Gleichung (3.75)):

$$
d K_{a}=d K_{b}=0 \text {. }
$$

Allerdings führt die Erhöhung der fiskalpolitischen Instrumenten-Indizes zu einem steigenden Zinssatz (vgl. Gleichung (3.76) in Verbindung mit Gleichung (3.63) bis Gleichung (3.65)):

$$
d r=\frac{\partial r}{\partial I_{a}} \cdot d I_{a}+\frac{\partial r}{\partial I_{b}} \cdot d I_{b}>0 .
$$

Anstatt also zusätzliches Kapital zu attrahieren, kommt die Erhöhung der fiskalpolitischen Instrumenten-Indizes den Kapitaleignern in Form einer höheren Rendite zu Gute. Die Regierungen haben durch ihr Verhalten lediglich den Nettozinssatz erhöht und ihr eigentliches Ziel, Kapital zu attrahieren, verfehlt. Dieser Vorgang ist in Abbildung 18 illustriert:

Kapitalnachfrage in Region a
Kapitalnachfrage in Region b

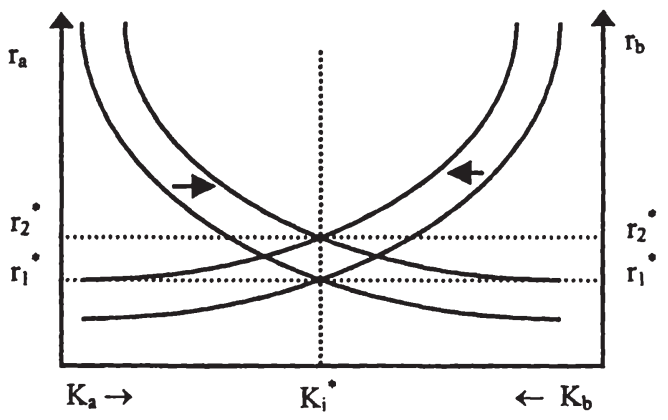

Abbildung 18: Wettbewerb beider Regionen um Kapital 
Wie in Abschnitt 3.3 heraus gearbeitet kann eine Niedrigsteuerregion ein höheres Niveau des Instrumenten-Indexes am einfachsten dadurch erreichen, indem sie die Menge an bereitgestelltem öffentlichen Input erhöht. Insofern ist bei Niedrigsteuerregionen damit zu rechnen, dass sie einen Ausgabenwettbewerb eingehen. Zusätzliche Ausgaben für das öffentliche Zwischenprodukt bewirken eine Erhöhung des Outputs der Region. Dieser zusätzliche Output kommt sowohl den abhängig Beschäftigten in Form eines höheren Lohns als auch den Kapitaleignern in Form einer höheren Nettorendite zu Gute. Hochsteuerregionen hingegen erzielen ein höheres Niveau des Instrumenten-Indexes am leichtesten durch Steuersatzsenkungen. Daher ist bei einer Föderation, die sich vorwiegend durch hohe Steuersätze auszeichnet, davon auszugehen, dass sie eher zu einem Steuersenkungswettbewerb neigt. Steuersenkungen, die das Ziel verfehlen, zusätzliches Kapital zu attrahieren, bewirken keine Veränderung des Outputs in der Region. Die steigende Nettorendite kann also nur das Resultat einer Umverteilung des Outputs sein. Durch die Senkung des Kapitalertragsteuersatzes entgeht dem Staat ein Teil des Outputs. Dieser Teil wird aufgrund des Steuersenkungswettbewerbs den Anteilseignern überlassen. Erfolgt nicht gleichzeitig eine Senkung der Lohnsteuersätze, so verschiebt sich das Verhältnis der Anteile der abhängig Beschäftigten und der Kapitaleigner am Gesamtoutput zu Gunsten der Kapitaleigner. Die hier skizzierte Wettbewerbspolitik verfehlt dann ihre beabsichtigte allokative Wirkung und führt eine unbeabsichtigte distributive Wirkung mit sich.

Halten die Regierungen nun in den Folgeperioden an ihrem Ziel fest, so setzt sich eine Spirale in Gang, die im ständigen Kampf um das Kapital letztlich zu einem permanent steigenden Nettozinssatz bei gleichbleibender Kapitalverteilung führt (siehe Abbildung (19)). Trotz dieses unerwünschten Resultats hat jedoch keine der Regierungen eine Veranlassung, ihr Verhalten im Alleingang zu ändern, da sie dann Gefahr läuft, dass aus ihrer Region letztlich noch Kapital abgezogen wird. Eine Lösung dieses Problems ist daher nur bei kooperativen Verhalten der Regierungen zu erwarten. Wie die Analyse über die Substituierbarkeit der fiskalpolitischen Instrumente gezeigt hat, ist es allerdings unbedingt erforderlich, dass diese kooperative Lösung die Wirkung beider fiskalpolitischer Instrumente berücksichtigt. 


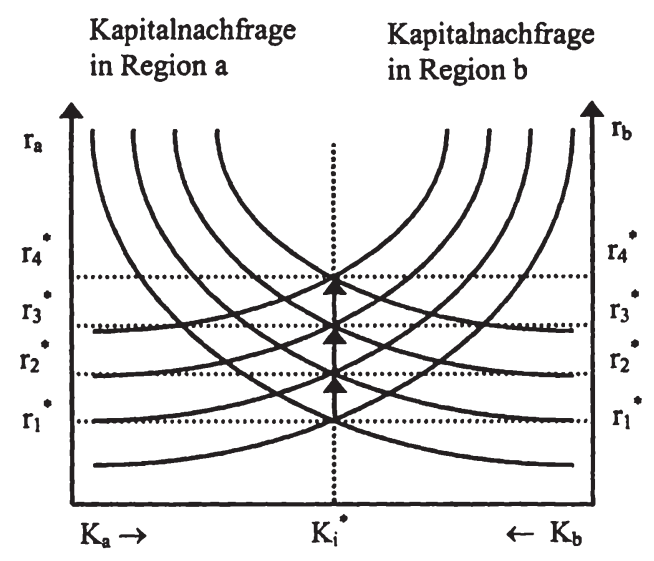

Abbildung 19: Wettbewerbsspirale mit permanent steigendem Nettozinssatz

\subsection{Zusammenfassung}

Abschließend lässt sich also festhalten, dass das in der Einleitung genannte Vorgehen der Bundesregierung in zweierlei Hinsicht problematisch ist.

Zum Ersten konnte gezeigt werden, dass sich die beiden fiskalpolitischen Instrumente gegeneinander substituieren lassen. Daher führt eine Steuersatzsenkung, die durch Senkung der Ausgaben für öffentliche Zwischenprodukte gegen finanziert wird, nicht ohne Weiteres zu einem höheren fiskalpolitischen Instrumenten-Index. Es besteht die Möglichkeit, dass die Steuersatzsenkung durch die Senkung der Menge an öffentlichem Input kompensiert oder gar überkompensiert wird. Es wurde verdeutlicht, dass ein höherer fiskalpolitischer Instrumenten-Index bei einer solchen Senkungspolitik um so eher zu erwarten ist, je höher das Niveau des Ausgangssteuersatzes der Region ausfällt. Je niedriger das Ausgangsniveau ist, um so wahrscheinlicher ist es, dass eine entsprechende Senkungspolitik einen niedrigeren fiskalpolitischen Instrumenten-Index bewirkt. Niedrigsteuerregionen können eher ein höheres Niveau des InstrumentenIndexes erreichen, indem sie die Ausgaben für öffentliche Inputs erhöhen und diese durch Steuersatzerhöhungen finanzieren.

Zum Zweiten garantiert jedoch selbst ein steigender Instrumenten-Index in einer Region keine erhöhte Kapitalmenge der selben Region. So hängt die letztlich 
von einer Region zusätzlich attrahierte Kapitalmenge nämlich von der Differenz der Änderungen der fiskalpolitischen Instrumenten-Indizes beider Regionen ab. Stimmen die Ziele der Regierungen einer Föderation in soweit überein, dass alle zusätzliches Kapital in ihre Regionen locken wollen, so entsteht eine ruinöse Wettbewerbsspirale. Letztlich erreicht dann keine der Regierungen ihr Ziel, sondern bewirkt durch ihr Verhalten lediglich, dass der Nettozinssatz ansteigt.

Es ist jedoch zu beachten, dass diese Ergebnisse nur mit Hilfe einer sehr speziellen Spezifikation der Zielfunktion der Regierungen erhalten wurden. Der Einfachheit halber wurde nämlich unterstellt, dass die Regierungen ausschließlich das Ziel verfolgen, zusätzliches Kapital zu attrahieren. Wesentlich realistischer wäre hingegen die Annahme, dass Regierungen darauf zielen, ihren Surplus zu maximieren und / oder ihre Wiederwahl zu sichern und dazu im gewissen Ausmaß zusätzliches Kapital attrahieren möchten. Eine entsprechende Untersuchung wird daher im folgenden Kapitel vorgenommen.

\section{Wirkung der Steuerharmonisierung auf Leviathanregierungen im Standortwettbewerb}

Das Thema „Steuerharmonisierung“ ist insbesondere aufgrund der Ausgestaltung der Steuerpolitik in der Europäischen Union derzeit von großer Bedeutung. Durch Artikel 93 des EG-Vertrages, der ein Harmonisierungsgebot für die Umsatzsteuer, die Verbrauchsabgaben und die sonstigen indirekten Steuern enthält, ist der Harmonisierungsprozess bezüglich der indirekten Steuern weit fortgeschritten.' So sind im Rahmen der Mehrwertsteuer die Bemessungsgrundlagen in der Europäischen Union nahezu harmonisiert und die übrigen indirekten Verbrauchsteuern teilweise angeglichen. ${ }^{2}$ Die direkten Steuern weisen demgegenüber auch heutzutage noch erhebliche nationale Unterschiede auf. ${ }^{3}$ Dies ist darauf zurückzuführen, dass im Gegensatz zu den indirekten Steuern keine EUVorschrift existiert, die eine Harmonisierung der direkten Steuern unmittelbar gebietet. ${ }^{4}$ Ein Harmonisierungsgebot der direkten Steuern lässt sich allenfalls durch eine entsprechende Interpretation der allgemeinen Rechtsangleichungs-

\footnotetext{
${ }^{1}$ Vgl. Pitlik (1998, S. 172).

${ }^{2} \mathrm{Vgl}$. Mans (1996, S. 331).

${ }^{3}$ Vgl. Mans (1996, S. 331), Pitlik (1998, S. 174).

${ }^{4}$ Vgl. Mans (1996, S. 334), Pitlik (1998, S. 173), Fleißig (2000, S. 133).
}

Claudia Hensberg - 978-3-631-75213-5 
vorschriften der Artikel 94 und 96 des EG-Vertrages konstruieren.' Insbesondere im Bereich der Zinsbesteuerung unternahm die Gemeinschaft immer wieder Bemühungen, eine Harmonisierung zu erreichen, die bisher allerdings stets nur zur weiteren Er- und Überarbeitung von Kompromissvorschlägen führten. ${ }^{2}$

Diese Unentschlossenheit der Europäischen Union spiegelt auch den gegenwärtigen kontroversen Forschungsstand der Wissenschaft bezüglich der Vorteilhaftigkeit einer Steuerharmonisierung der direkten Steuern wider. Befürworter einer Steuerharmonisierung nennen als Vorteil, dass durch eine abgestimmte Steuerpolitik der ruinöse Steuersenkungswettlauf zwischen den Mitgliedstaaten eingedämmt werden kann. ${ }^{3}$ Gegner verweisen darauf, dass die Steuerharmonisierung den Wettbewerb zwischen Regierungen unterbindet, durch den eine fiskalische Disziplinierung der Regierungen erzeugt werden soll. ${ }^{4}$ In dieser Diskussion um die Vorteilhaftigkeit der Steuerharmonisierung wird die Aufmerksamkeit in der Regel ausschließlich auf die Auswirkungen der Steuerharmonisierung bezüglich des Steuerwettbewerbs und der damit verbundenen Problematik gelenkt.

In diesem Kapitel sollen hingegen mögliche Auswirkungen der Steuerharmonisierung auf die Ausgabenpolitik der Regierungen auf theoretischer Ebene modelliert werden. Dazu wird unterstellt, dass die einzelnen Gebietskörperschaften einer Föderation zwar durch die Steuerharmonisierungsvorschriften an die gemeinschaftliche Steuerpolitik gebunden sind, dass sie aber in ihrer Ausgabenpolitik vollkommen autonom sind. Obwohl die einzelnen Regierungen somit die Steuerpolitik nicht beeinflussen können und diese daher auch nicht im Standortwettbewerb zur Attrahierung von Produktionsfaktoren benutzen können, steht den Regierungen die Möglichkeit offen, durch eine entsprechende Ausgabenpolitik ihren Standort attraktiv zu gestalten.

Dieses Kapitel ist wie folgt strukturiert: In Abschnitt 4.1 wird zunächst das Modell des Ausgabenwettbewerbs vorgestellt. Dabei werden die Regierungen als Leviathanregierungen modelliert, die sich in einem nicht-kooperativen NashSpiel befinden. In Abschnitt 4.2 werden die Reaktionsfunktionen der Leviathanregierungen aus dem Surplus maximierenden Verhalten der Regierungen hergeleitet. Darauf aufbauend werden in Abschnitt 4.3 die Bedingungen für ein Nash-

\footnotetext{
${ }^{1}$ Vgl. Sapusek (1997, S. 306), Pitlik (1998, S. 173).

${ }^{2} \mathrm{Vgl}$. Fleißig (2000, S. $143 \mathrm{ff}$.).

${ }^{3}$ Vgl. Pitlik (1998, S. 170).

${ }^{4}$ Vgl. Pitlik (1998, S. 170).
} 
Gleichgewicht herausgearbeitet. Abschnitt 4.4 setzt sich dann mit den Auswirkungen des harmonisierten Steuersatzes auf die im Gleichgewicht für den Leviathan optimale Menge an öffentlichem Input auseinander. Das Kapitel endet mit einer Zusammenfassung in Abschnitt 4.5.

\subsection{Das Modell}

Betrachtet wird eine Föderation, die sich aus zwei gleichen Regionen ( $a$ und $b$ ) zusammensetzt. In jeder Region werden die beiden privaten Primärfaktoren, Arbeit (L) und Kapital (K), zur Produktion eingesetzt. Jede Region ist mit einer exogen vorgegebenen Anzahl an nicht mobilen Arbeitskräften $\left(\bar{L}_{a}\right.$ bzw. $\left.\bar{L}_{b}\right)$ ausgestattet. ${ }^{1}$ Das Kapital wird als vollständig mobil unterstellt. ${ }^{2}$ Mit Hilfe der privaten Primärfaktoren produzieren die Unternehmen, deren Anzahl exogen vorgegeben ist, ein Einheitsgut (Y). Dieses Einheitsgut dient als numéraire mit einem Preis von Eins. ${ }^{3}$

\subsubsection{Die Regierung als Leviathan}

Im Gegensatz zur herrschenden Theorie ${ }^{4}$ werden die Regierungen nicht als „wohlwollend" modelliert. Vielmehr wird der von Brennan und Buchanan begründeten Tradition gefolgt und unterstellt, dass die Regierungen ihren eigenen Nutzen maximieren. ${ }^{5}$ Der Nutzen bestimmt sich ausschließlich in Abhängigkeit des Finanzierungsüberschusses, den die Regierung erwirtschaftet. Der Finanzierungsüberschuss (S) ist definiert als die Differenz zwischen den Steuereinnahmen $(\mathrm{T})$ und den Staatsausgaben $(\mathrm{C}):^{6}$

$$
\text { (4.1) } \mathrm{S}=\mathrm{T}-\mathrm{C} \text {. }
$$

\footnotetext{
' Vgl. Tausch (1998, S. 330).

${ }^{2}$ Vgl. Oates / Schwab (1988, S. 337), Edwards / Keen (1996, S. 117), Sinn (1997, S. 14 und 18), Rauscher (1998, S. 61), Tausch (1998, S. 330). Zum Realitätsbezug dieser Annahme siehe auch Apolte (1999, S. 24 ff.).

${ }^{3}$ Vgl. Wildasin (1988, S. 231), Tausch (1998, S. 330).

${ }^{4}$ Vgl. beispielsweise Zodrow / Mieszkowski (1986), Oates / Schwab (1988), Sinn (1997), Tausch (1998).

${ }^{5}$ Vgl. Brennan / Buchanan (1980, S. 169).

${ }^{6} \mathrm{Vgl}$. Brennan / Buchanan (1980, S. 27 und 169), Niskanen (1997, S. 466).
} 
Die Höhe der Steuereinnahmen kann die jeweilige lokale Regierung mit Hilfe ihres Steuermonopols nicht direkt beeinflussen. Die einzelnen Regierungen werden nämlich durch eine Finanzverfassung' in ihrem Steuermonopol beschränkt. Diese Finanzverfassung schreibt vor, dass die Regierungen alleinig eine Substanzsteuer auf das Kapital erheben können. Die Steuereinnahmen einer Region $\mathrm{i}^{2}\left(T_{i}\right)$ ergeben sich demnach als Produkt aus dem Steuersatz der Region $\left(t_{K i}\right)$ und dem in der Region eingesetzten Kapital $\left(K_{i}\right)$ :

$$
T_{i}=t_{K i} \cdot K_{i} \text {. }
$$

Die Finanzverfassung legt darüber hinaus fest, dass die Steuern in der gesamten Föderation harmonisiert sind. Zum einen bedeutet dies, dass die Bemessungsgrundlagen nach denselben Vorschriften ermittelt werden. ${ }^{3}$ Zum anderen gelten aber auch in allen Regionen einheitliche Steuersätze, ${ }^{4}$ so dass der Kapitalsteuersatz einer jeden Region dem harmonisierten Kapitalsteuersatz $\left(\tilde{t}_{K}\right)$ entspricht:

$$
t_{K i}=\tilde{t}_{K} \text {. }
$$

Die gesamten Steuereinnahmen einer Region $\left(\mathrm{T}_{\mathrm{i}}\right)$ belaufen sich daher auf:

$$
T_{i}=\tilde{t}_{K} \cdot K_{i} \text {. }
$$

${ }^{1}$ Das Konzept der Finanzverfassung als Beschränkung des Leviathanstaates von Brennan und Buchanan geht auf die Überlegungen von Hobbes zurück. Dieser ging von einem hypothetischen Ursprungszustand der Anarchie aus, der sich durch hohe Kosten der Unsicherheit auszeichnet. Gegenüber diesem Ursprungszustand ist es dann gesamtwirtschaftlich effizienter, einen ubergeordneten Machthaber - den Leviathan - einzusetzen, damit zumindest ein gewisses $\mathrm{Maß}$ an öffentlichen Gütern - insbesondere das öffentliche Gut „Sicherheit" - bereitgestellt wird. Es ist die hierin enthaltene Idee des Gesellschaftsvertrages, an die Brennan und Buchanan anknüpfen. Sie nehmen an, dass rationale Individuen ,hinter dem Schleier der Ungewissheit" ex ante eine Finanzverfassung aufstellen, die das Ausmaß des Steuermonopols des Leviathans verbindlich festlegt (vgl. Brennan / Buchanan (1980, S. 33)). Darauf aufbauend gehen sie dann der Frage nach, wie eine solche Finanzverfassung effizienter Weise zu gestalten ist.

Die Annahme einer dem Leviathan vorgegebenen Finanzverfassung ist jedoch sowohl hinsichtlich der Entstehung einer solchen Verfassung als auch hinsichtlich der Durchsetzung nicht unproblematisch. Da in diesem Kapitel allerdings der Leviathanstaat ohnehin nur als entscheidungstheoretische Referenzfigur verwendet wird, soll das Konzept der Finanzverfassung, wie es Brennan und Buchanan vorschlagen, als theoretisches Konstrukt in das hier präsentierte Modell eingehen.

${ }^{2}$ Region i repräsentiert wahlweise Region a oder Region b. Das Symbol i ist demnach als Variable für $\mathrm{a}$ und $\mathrm{b}$ zu verstehen.

${ }^{3}$ Vgl. Pitlik (1998, S. 170).

${ }^{4} \mathrm{Vgl}$. Kamada (1989, S. 4), Homburg (1999, S. 9). 
Da die Steuersätze von der Finanzverfassung fest vorgegeben sind, kann die einzelne Regierung also nur indirekt über die Menge an attrahiertem Kapital die Höhe der Steuereinnahmen beeinflussen.

Das Instrument, welches den Regierungen hierfür zur Verfügung steht, ist ein öffentliches Zwischenprodukt. Die Strategiemenge einer jeden Regierung, d.h. die Menge an öffentlichem Zwischenprodukt, sei eine konvexe und kompakte Teilmenge von $\mathrm{IR}_{0}^{+}{ }^{+}$' Das öffentliche Zwischenprodukt ist ein lokal öffentliches Gut, so dass die Nutzung des öffentlichen Zwischenproduktes durch gebietsfremde Unternehmen ausgeschlossen ist. ${ }^{2}$ Die Unternehmen können es, ohne dass ihnen dabei direkte Kosten entstehen, nutzen. Des Weiteren zeichnet sich das öffentliche Zwischenprodukt durch Nicht-Rivalität im Konsum aus und ist somit ein rein öffentliches Gut im Sinne von Samuelson. ${ }^{3}$ Insofern handelt es sich um ein „factor-augmenting public input" ${ }^{4}{ }^{4}$ Das öffentliche Zwischenprodukt ist Hicks-neutral, weshalb es die gleiche Wirkung auf beide privaten Primärfaktoren entfaltet. ${ }^{5}$ Es führt zu einem Anstieg des Outputs des Einheitsgutes und wirkt insofern produktivitätssteigernd. ${ }^{6}$ Die Wirkung des öffentlichen Zwischenprodukts im Produktionsprozess kann durch einen Effizienzparameter (E) dargestellt werden. ${ }^{7}$ Der Effizienzparameter hängt von der Höhe der bereitgestellten Menge an öffentlichem Input ab:

$$
E_{i}=E\left(G_{i}\right) \text {. }
$$

Wird das öffentliche Zwischenprodukt nicht angeboten, dann nimmt dieser $\mathrm{Pa}$ rameter den Wert Eins an:

$$
E(0)=1 \text {. }
$$

Mit steigendem Einsatz des Zwischenprodukts steigt der Wert des Parameters derart an, dass

$$
\frac{d E}{d G_{i}}>0
$$

und

\footnotetext{
${ }^{1}$ Vgl. Friedman (1977, S. 152), Holler / Illing (1991, S. 33 f. und 67).

${ }^{2}$ Vgl. Richter / Wellisch (1993, S. 438).

${ }^{3}$ Vgl. Samuelson (1954).

${ }^{4}$ Vgl. Kaizuka (1965, S. 118 ff.), Tausch (1998, S. 331), Colombier (2001, S. 43 ff.).

${ }^{5}$ Vgl. Colombier (2001, S. 45).

${ }^{6} \mathrm{Vgl}$. Tausch (1998, S. 331).

${ }^{7}$ Vgl. Tausch (1998, S. 331), Roloff (1999, S. 50; 2001, S. 116 f.). 


$$
\frac{d^{2} E}{d G_{i}^{2}}<0
$$

vorliegt.

Der Einfachheit halber wird unterstellt, dass das öffentliche Zwischenprodukt außerhalb der Föderation produziert wird und die einzelnen Regionen es von dort erwerben. ${ }^{1}$ Die Kosten (C), die einer Region dabei entstehen, hängen von der Menge an bereitgestelltem öffentlichen Zwischenprodukt ab:

$$
C_{i}=C\left(G_{i}\right) \text {. }
$$

Mit zunehmendem Einsatz des öffentlichen Zwischenprodukts steigen diese an:

$$
\frac{d C}{d G_{i}}>0 \text {. }
$$

Die Kostenfunktion ist derart strukturiert, dass die 2. Ableitung der Kostenfunktion größer ist als die 2. Ableitung der Einnahmenfunktion des Staates. ${ }^{2}$ Ferner gelte: ${ }^{3}$

$$
\text { (4.11) } \frac{d^{2} C}{d G_{i}^{2}}>0 \text {. }
$$

Die Technologie zur Produktion des öffentlichen Gutes weist somit abnehmende Skalenerträge auf. ${ }^{4}$

\subsubsection{Die Regierungen im nicht- kooperativen Nash-Spiel}

Der Handlungsspielraum der Regierungen wird außer durch die Finanzverfassung auch durch die Reaktionen auf den privaten Märkten begrenzt. Da die Regierungen lediglich eine Kapitalsteuer erheben, ist für diese auch nur der Kapitalmarkt von Interesse. Auf dem Kapitalmarkt treten die Unternehmen als Nach-

\footnotetext{
${ }^{1}$ Vgl. Apolte (2001, S. 361).

${ }^{2}$ Vgl. Friedman (1983, S. 30).

${ }^{3}$ In der herrschenden Literatur (vgl. beispielsweise Sinn (1997)) wird die Annahme einer Kostenfunktion mit den genannten Eigenschaften stets durch Stauungskosten gerechtfertigt. Wie Colombier (2001) und Colombier / Pickhardt (1999) jedoch gezeigt haben, ist die Annahme von Stauungskosten mit der Annahme eines 8 ffentlichen Zwischenproduktes im Sinne von Samuelson unvereinbar. Daher wird in dieser Arbeit die Modellierung von Stauungskosten unterlassen.

${ }^{4}$ Eine solche Annahme kann beispielsweise mit Ineffizienzen im öffentlichen Sektor begründet werden.
} 
frager auf. Diese verfolgen das Ziel, ihren Gewinn (П) zu maximieren. Letzterer setzt sich aus den Einnahmen, die aufgrund eines Preises von Eins dem Output entsprechen, abzüglich der Kosten zusammen. Der Output (Y) einer Region wird durch die eingesetzte Menge an Kapital (K), Arbeit (L) und öffentlichem Zwischenprodukt (G) bestimmt. Die Produktionsfunktion einer Region (i) stellt sich somit dar als:

$$
Y_{i}=E\left(G_{i}\right) \cdot f_{i}\left(K_{i}, \bar{L}_{i}\right),
$$

wobei die Grenzproduktivität des Kapitals positiv und abnehmend ist:

$$
\begin{aligned}
& \frac{\partial f_{i}\left(K_{i}, \bar{L}_{i}\right)}{\partial K_{i}} \equiv f_{K i}\left(K_{i}\right)>0, \\
& \frac{\partial^{2} f_{i}\left(K_{i}, \bar{L}_{i}\right)}{\partial K_{i}^{2}} \equiv f_{K i K_{i}}\left(K_{i}\right)<0 .
\end{aligned}
$$

Bei den Kosten, die den Unternehmen entstehen, handelt es sich zum einen um den Lohn für die benötigten Arbeitskräfte und zum anderen um Zinszahlungen für das eingesetzte Kapital. Die Gewinnfunktion der Unternehmen einer Region (i) entspricht somit:

$$
\Pi_{i}=E\left(G_{i}\right) \cdot f_{i}\left(K_{i}, \bar{L}_{i}\right)-z_{i} \cdot K_{i}-w_{i} \cdot \bar{L}_{i},
$$

wobei $\mathrm{z}$ den Bruttozinssatz und $\mathrm{w}$ den Bruttolohnsatz repräsentiert. Die Unternehmer maximieren ihren Gewinn u.a., wenn die Grenzproduktivität des Kapitals mit dem Bruttozinssatz übereinstimmt:

$$
E\left(G_{i}\right) \cdot f_{K i}\left(K_{i}\right)=z_{i} \text {. }
$$

Die Kapitaleigner legen ihr Kapital in der Region an, in der sie den höchsten Nettozinssatz erzielen können. ${ }^{1} \mathrm{Da}$ das Kapital innerhalb der Föderation vollkommen mobil ist, bildet sich ein einheitlicher Nettozinssatz (r) in der Föderation: ${ }^{2}$

$$
r=r_{i} \text {. }
$$

Da die Kapitalsteuersätze darüber hinaus harmonisiert sind, sind auch die Bruttozinssätze in den Regionen identisch, so dass in der gesamten Föderation ein einheitlicher Bruttozinssatz (z) vorliegt:

$$
z=z_{i} \text {. }
$$

\footnotetext{
${ }^{1}$ Vgl. Oates / Schwab (1988, S. 337).

${ }^{2}$ Vgl. Zodrow / Mieszkowski (1986, S. 358), Wildasin (1991, S. 397), Edwards / Keen (1996, S. 117), Apolte (2001, S. 361).
} 
Der Kapitalbestand der Föderation $(\bar{K})$ ist exogen vorgegeben und fix. ${ }^{\prime}$ Im Gleichgewicht müssen daher bei einheitlichem Netto- bzw. Bruttozinssatz die in den beiden Regionen nachgefragten Kapitalmengen dem Kapitalbestand entsprechen:

$$
K_{a}+K_{b}=\bar{K} \text {. }
$$

Aus Gleichung (4.16), Gleichung (4.18) und Gleichung (4.19) erhält man somit die Gleichgewichtsbedingung für den Kapitalmarkt:

$$
E\left(G_{a}\right) \cdot f_{K a}\left(K_{a}\right)=z=E\left(G_{b}\right) \cdot f_{K b}\left(\bar{K}-K_{a}\right)
$$

bzw.

$$
E\left(G_{b}\right) \cdot f_{K b}\left(K_{b}\right)=z=E\left(G_{a}\right) \cdot f_{K a}\left(\bar{K}-K_{b}\right) .
$$

Bedingung (20) ist eine implizite Funktion der Kapitalmenge $\mathrm{K}_{\mathrm{a}}$ bzw. Bedingung (21) eine implizite Funktion der Kapitalmenge $K_{b}$. Es zeigt sich, dass die Kapitalmenge in der jeweiligen Region sowohl von der Höhe der bereitgestellten Menge an öffentlichem Zwischenprodukt in Region a als auch von der Höhe der Menge an öffentlichem Zwischenprodukt in Region b abhängt. Die Höhe des Surplus einer Regierung wird also nicht nur durch die eigenen Entscheidungen bestimmt, sondern auch durch das Verhalten der Konkurrenzregierung beeinflusst. Die Situation, in der die Regierungen sind, ist somit eine Analogie zu der, in der sich die Oligopolisten im Cournot-Modell befinden. ${ }^{2}$ Es handelt sich also um ein Nash-Cournot-Spiel, welches die Regierungen eingehen.

Dieses Spiel ist wie folgt charakterisiert: Die Höhe des Surplus einer jeden Regierung hängt von den Entscheidungen beider Regierungen $a b .^{3}$ Die Regierungen sind sich der Interdependenz bewusst und sie gehen davon aus, dass die jeweilige Konkurrenzregion sich auch dieser Interdependenz bewusst ist. ${ }^{4} \mathrm{Die} \mathrm{Re}-$ gierungen handeln rational und haben vollständige Information über sich selbst, die Märkte und die Konkurrenzregierungen. ${ }^{5}$ Die Finanzverfassung verbietet sowohl bindende Vereinbarungen als auch informelle Absprachen. ${ }^{6}$ Eine Kommunikation zwischen den beiden Regierungen, die eine Koordination der Strategien bewirkt, ist somit ausgeschlossen. ${ }^{7}$ Es handelt sich demnach um ein nicht-

\footnotetext{
${ }^{1} \mathrm{Vgl}$. Hensberg (2002).

${ }^{2}$ Vgl. Cournot (1838), Friedman (1983, S. 19 ff.), Heap / Varoufakis (1995, S. 54 ff.).

${ }^{3}$ Vgl. Holler / Illing (1991, S. 1), Rieck (1993, S. 16).

${ }^{4} \mathrm{Vgl}$. Holler / Illing (1991, S. 1).

${ }^{5}$ Vgl. Friedman (1977, S. 17), Holler / Illing (1991, S. 45 und 64).

${ }^{6} \mathrm{Vgl}$. Friedman (1977, S. 23), Rieck (1993, S. 28).

${ }^{7}$ Vgl. Holler / Illing (1991, S. 3).
} 
kooperatives Spiel. ${ }^{1}$ Die Lösung eines solchen Spiels existiert, sofern keine der Regierungen einen Anreiz hat, von dieser Lösung abzuweichen. ${ }^{2}$ Eine Lösung kann also nur existieren, wenn beide Regierungen ihr Ziel, ihren Surplus zu maximieren, gleichzeitig erreichen. Die Strategiewahl zur Erreichung des Ziels besteht dabei darin, dass jeder Spieler über seine eigene Strategie entscheidet unter der Annahme, dass die übrigen Spieler ihren Surplus maximieren.

\subsection{Das Surplusmaximum der Regierungen}

Die Höhe des Surplus $\left(\mathrm{S}_{\mathrm{i}}\right)$ hängt von der Höhe des harmonisierten Steuersatzes $\left(\tilde{t}_{K}\right)$, der in der jeweiligen Region (i) eingesetzten Kapitalmenge $\left(\mathrm{K}_{\mathrm{i}}\right)$ und von den Kosten (C), die bei der Bereitstellung des öffentlichen Zwischenproduktes $\left(\mathrm{G}_{\mathrm{i}}\right)$ anfallen, $\mathrm{ab}$ :

$$
S_{i}=\tilde{t}_{K} \cdot K_{i}-C\left(G_{i}\right)
$$

(vgl. Gleichung (4.1), Gleichung (4.4) und Gleichung (4.9)). Die einzelnen Regierungen können den Surplus allein über die Menge an bereitgestelltem öffentlichen Input beeinflussen. Dabei haben sie die Wirkung des öffentlichen Zwischenproduktes auf dem Kapitalmarkt zu berücksichtigen:

$$
S_{i}=\tilde{t}_{K} \cdot K_{i}\left(G_{i}\right)-C\left(G_{i}\right) \text {. }
$$

Die Abhängigkeit des Surplus von der Menge an öffentlichem Zwischenprodukt ist in Abbildung (20) beispielhaft, grafisch dargestellt. Jede der Leviathanregierungen strebt nun danach, ihren Surplus zu maximieren. Leitet man Gleichung (4.23) nach $G_{i} a b$, so erhält man als notwendige Bedingung für ein Surplusmaximum:

$$
\frac{d S_{i}}{d G_{i}}=\widetilde{t}_{K} \cdot \frac{\partial K_{i}}{\partial G_{i}}-\frac{d C}{d G_{i}}=0 .
$$

Die hinreichende Bedingung ist erfüllt, da annahmegemäß die 2. Ableitung der Kostenfunktion größer ist als die 2. Ableitung der Einnahmenfunktion. Die Leviathanregierungen maximieren also ihren Surplus, wenn die Grenzeinnahmen den Grenzkosten entsprechen:

\footnotetext{
1 Vgl. Friedman (1977, S. 17), Holler / Illing (1991, S. 3), Rieck (1993, S. 29), Sinn (1997, S. 12).

${ }^{2}$ Vgl. Holler / Illing (1991, S. 6).
} 


$$
\tilde{t}_{K} \cdot \frac{\partial K_{i}}{\partial G_{i}}=\frac{d C}{d G_{i}}
$$

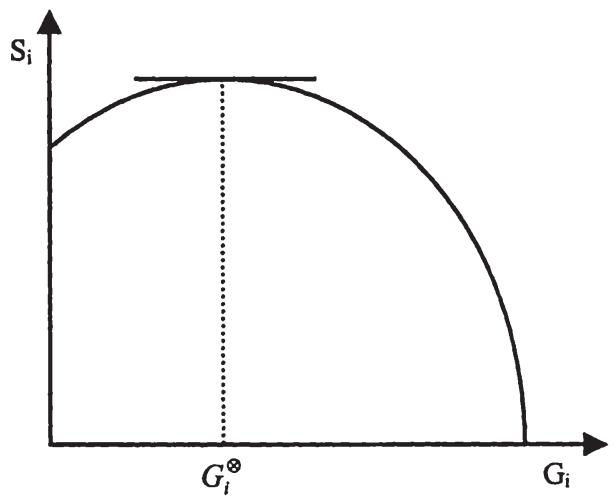

Abbildung 20: Die Surplusfunktion einer Leviathanregierung

Bedingung (4.25) ist eine implizite Funktion der für die Regierungen optimalen Menge an öffentlichem Zwischenprodukt $\left(G_{i}^{\otimes}\right)$ und stellt somit die Reaktionsfunktion der Leviathanregierung i dar. Die optimale Menge an öffentlichem Input hängt also maßgeblich von der durch die Bereitstellung des öffentlichen Zwischenprodukts zusätzlich attrahierten Menge an Kapital ab. Wie viel Kapital zusätzlich attrahiert wird, wird durch den Kapitalmarkt festgelegt. Der Kapitalmarkt befindet sich im Gleichgewicht, wenn

$$
E\left(G_{a}\right) \cdot f_{K a}\left(K_{a}\right)=E\left(G_{b}\right) \cdot f_{K b}\left(\bar{K}-K_{a}\right)
$$

gilt (vgl. Gleichung (4.20)). Leitet man diese implizite Funktion nach $\mathrm{G}_{\mathrm{a}}$ ab, so ergibt sich:"

$$
\frac{\partial K_{a}}{\partial G_{a}}=\frac{d E}{d G_{a}} \cdot \frac{f_{K a}\left(K_{a}\right)}{-\left(E\left(G_{a}\right) \cdot f_{K a K a}\left(K_{a}\right)+E\left(G_{b}\right) \cdot f_{K b K b}\left(K_{b}\right)\right)}>0 .
$$

Durch Ableitung der Funktion nach $\mathrm{G}_{\mathrm{b}}$ erhält man entsprechend:

$$
\frac{\partial K_{b}}{\partial G_{b}}=\frac{d E}{d G_{b}} \cdot \frac{f_{K b}\left(K_{b}\right)}{-\left(E\left(G_{a}\right) \cdot f_{K a K a}\left(K_{a}\right)+E\left(G_{b}\right) \cdot f_{K b K b}\left(K_{b}\right)\right)}>0 .
$$

\footnotetext{
'Für die genaue Berechnung siehe Anhang 8.
} 
Somit kann die Reaktionsfunktion der Leviathanregierung a zu

$$
\tilde{t}_{K} \cdot \frac{d E}{d G_{a}} \cdot \frac{f_{K a}\left(K_{a}\right)}{-\left(E\left(G_{a}\right) \cdot f_{K a K a}\left(K_{a}\right)+E\left(G_{b}\right) \cdot f_{K b K b}\left(K_{b}\right)\right)}=\frac{d C}{d G_{a}}
$$

(siehe Gleichung (4.25) und Gleichung (4.27)) und die der Regierung b zu

$$
\tilde{t}_{K} \cdot \frac{d E}{d G_{b}} \cdot \frac{f_{K b}\left(K_{b}\right)}{-\left(E\left(G_{a}\right) \cdot f_{K a K a}\left(K_{a}\right)+E\left(G_{b}\right) \cdot f_{K b K b}\left(K_{b}\right)\right)}=\frac{d C}{d G_{b}}
$$

(siehe Gleichung (4.25) und Gleichung (4.28)) konkretisiert werden.

$\mathrm{Da}$ die 2. Ableitung der Surplusfunktion für $\mathrm{G}_{\mathrm{i}} \in \mathrm{IR}_{0}^{+}$kleiner null ist, kann höchstens eine Lösung der Gleichung (4.29) bzw. der Gleichung (4.30) für $G_{a}$ bzw. $G_{b}$ vorliegen.' Allerdings ist es somit auch möglich, dass die Gleichungen (4.29) und (4.30) für $\mathrm{G}_{\mathrm{i}} \in \mathrm{IR}_{0}{ }^{+}$nicht lösbar sind. Die Surplusfunktion der jeweiligen Regierung besitzt dann kein globales Maximum (siehe Abbildung 21).

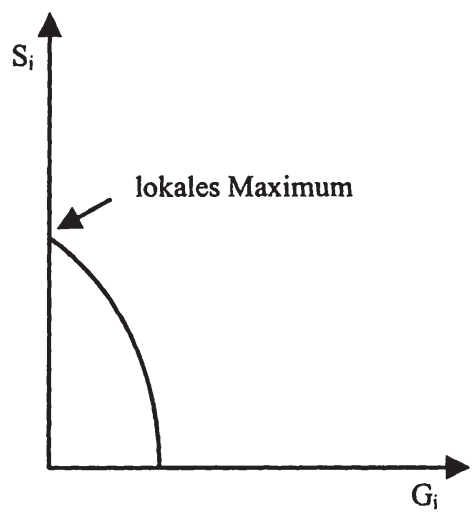

Abbildung 21: Lokales Maximum einer Surplusfunktion

In diesem Fall (Fall 1) kann die Regierung ein Surplusmaximum nur erreichen, indem sie das lokale Maximum wählt. Das lokale Maximum besteht - wie Abbildung 21 veranschaulicht - bei $\mathrm{G}_{\mathrm{i}}=0$. Die Leviathanregierungen werden dann also davon absehen, das öffentliche Zwischenprodukt bereitzustellen. Die Regierungen werden ebenfalls die Bereitstellung des öffentlichen Inputs unterlassen, falls ein globales Maximum bei $\mathrm{G}_{\mathrm{i}}=0$ gegeben ist (Fall 2) (vgl. Abbildung 22).

${ }^{1}$ Für den Beweis dieser Behauptung siehe Anhang 9. 


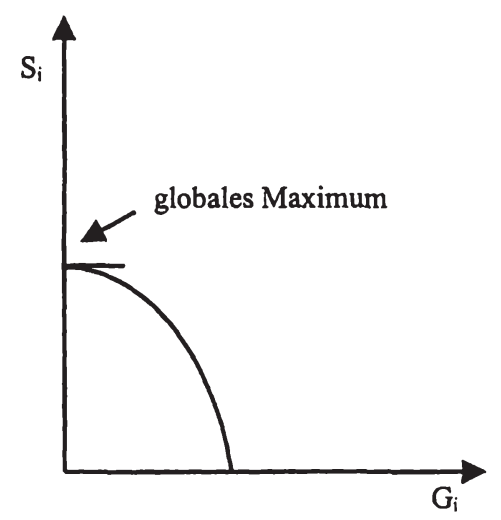

Abbildung 22: Globales Maximum einer Surplusfunktion bei $G_{i}=0$

Neben diesen Randlösungen kann das globale Maximum aber auch im Bereich $\mathrm{G}_{\mathrm{i}} \in \mathrm{IR}^{+}$liegen (Fall 3) (siehe Abbildung 20). In diesem Fall haben die Regierungen einen eigenständigen finanziellen Anreiz, das öffentliche Zwischenprodukt bereitzustellen.' So wird durch eine zusätzlich bereitgestellte Menge an öffentlichem Zwischenprodukt Kapital in die Region gelockt, wodurch die Bemessungsgrundlage der Kapitalsubstanzsteuer verbreitert wird und die Einnahmen der Leviathanregierung erhöht werden. Diesen zusätzlichen Einnahmen stehen zusätzliche Kosten, die durch die Bereitstellung des öffentlichen Zwischenproduktes erzeugt werden, gegenüber. Die jeweilige Leviathanregierung wird also ihr Angebot an öffentlichem Input so weit ausdehnen, bis sich Grenzertrag und Grenzkosten desselben entsprechen.

$\mathrm{Ob}$ und wie viel öffentlichen Input die Regierungen bereitstellen, hängt nun von folgenden Faktoren ab: Zum Ersten üben die Technologien Einfluss auf die für die Regierungen optimale Menge an öffentlichem Zwischenprodukt aus. So beeinflusst die Art der Produktionsfunktion (f) die Grenzproduktivität des Kapitals und insofern auch die Höhe der durch eine zusätzliche Einheit an öffentlichem Input attrahierten Kapitalmenge. Je nach Art des Effizienzparameters (E) entfaltet das öffentliche Zwischenprodukt eine andere Wirkung im Produktionsprozess und bestimmt darüber die Menge an Kapital, die von den Unternehmen in einer Region eingesetzt wird. Aber auch der Markt für das öffentliche Zwischenprodukt ist relevant, da durch ihn die Kostenfunktion (C) festgelegt wird.

${ }^{1}$ Vgl. Roloff (2001, S. 138 f.). 
Die Einflüsse, die von den Technologien ausgehen, seien im Weiteren durch die Funktion $\Phi$ repräsentiert. Zum Zweiten spielt der Harmonisierungssteuersatz $\left(\tilde{t}_{K}\right)$ eine entscheidende Rolle bezüglich der für den Leviathan optimalen Menge an öffentlichem Zwischenprodukt. Auf seine Wirkung soll in Abschnitt 4.4 noch genauer eingegangen werden. Zum Dritten ist jedoch auch das Verhalten der Konkurrenzregion maßgeblich für die Menge an öffentlichem Input, die eine Regierung bereitstellen wird. Je nach Höhe der von der Konkurrenzregion angebotenen Menge an öffentlichem Zwischenprodukt $\left(G_{b}\right.$ bzw. $\left.G_{a}\right)$ ist für die Leviathanregierung eine andere Menge optimal. Unter Beachtung dieser Überlegungen lässt sich die Reaktionsfunktion aus Gleichung (4.29) bzw. Gleichung (4.30) auch explizit als

$$
G_{a}^{\otimes}=\Phi_{a}^{1}\left(G_{b}, \tilde{t}_{K}\right)
$$

bzw.

$$
G_{b}^{\otimes}=\Phi_{b}^{1}\left(G_{a}, \tilde{t}_{K}\right)
$$

schreiben. In Abhängigkeit von der Menge an öffentlichem Zwischenprodukt der Konkurrenzregion $\left(G_{b}\right.$ bzw. $\left.G_{a}\right)$, dem harmonisierten Steuersatz $\left(\tilde{t}_{K}\right)$ und den Technologien ( $\Phi_{a}^{1}$ bzw. $\Phi_{b}^{1}$ ) ergibt sich demnach eine eindeutige Lösung der für die jeweilige Regierung optimalen Menge an öffentlichem $\operatorname{Input}\left(G_{i}^{\otimes}\right)$.

\subsection{Das Nash-Gleichgewicht}

Ein Nash-Gleichgewicht liegt vor, wenn jede Regierung ihren Finanzierungsüberschuss unter der Annahme maximiert, dass die anderen Regierungen ebenfalls ihren Finanzierungsüberschuss maximieren. ${ }^{\prime}$ Das Nash-Gleichgewicht ist ein stabiles Gleichgewicht, da sich kein Spieler verbessern kann, wenn er alleine von der Gleichgewichtskombination abweicht. ${ }^{2}$ Ausgangspunkt ist das individuelle Surplusmaximum einer jeden Regierung (vgl. Gleichung (4.29) bzw. Gleichung (4.30)). Der Einfachheit halber wurde die Reaktionsfunktion der Regierung a umgeformt $\mathrm{zu}$ :

${ }^{1}$ Vgl. Nash (1951), Holler / Illing (1991, S. 60).

${ }^{2}$ Vgl. Friedman (1977, S. 149), Rieck (1993, S. 25). 


$$
\frac{\left(\frac{d E}{d G_{a}}\right)}{\left(\frac{d C}{d G_{a}}\right)} \cdot f_{K a}\left(K_{a}\right) \cdot \frac{\tilde{t}_{K}}{-\left(E\left(G_{a}\right) \cdot f_{K a K a}\left(K_{a}\right)+E\left(G_{b}\right) \cdot f_{K b K b}\left(K_{b}\right)\right)}=1
$$

und die der Regierung b zu:

$$
\frac{\left(\frac{d E}{d G_{b}}\right)}{\left(\frac{d C}{d G_{b}}\right)} \cdot f_{K b}\left(K_{b}\right) \cdot \frac{\tilde{t}_{K}}{-\left(E\left(G_{a}\right) \cdot f_{K a K a}\left(K_{a}\right)+E\left(G_{b}\right) \cdot f_{K b K b}\left(K_{b}\right)\right)}=1 .
$$

Im Gleichgewicht müssen nun beide Regierungen gleichzeitig ihr Surplusmaximum erreichen, d.h. beide Bedingungen (Gleichung (4.33) und Gleichung (4.34)) müssen gleichzeitig erfüllt sein. ${ }^{1}$ Ob dies möglich ist, lässt sich ermitteln, indem die beiden Bedingungen gleichgesetzt werden:

$$
\frac{\left(\frac{d E}{d G_{a}}\right)}{\left(\frac{d C}{d G_{a}}\right)} \cdot f_{K a}\left(K_{a}\right)=\frac{\left(\frac{d E}{d G_{b}}\right)}{\left(\frac{d C}{d G_{b}}\right)} \cdot f_{K b}\left(K_{b}\right) \text {. }
$$

Es liegt demnach ein Nash-Gleichgewicht vor, wenn das Verhältnis von marginaler Effizienz zu marginalen Kosten des öffentlichen Zwischenproduktes, gewichtet mit der Grenzproduktivität des Kapitals, bei Region a und bei Region b übereinstimmt.

Eine Lösung für Gleichung (4.35) besteht, wenn in beiden Regionen die gleiche, für die Regierungen optimale Menge an öffentlichem Input $\left(G_{i}^{\otimes}\right)$ bereitgestellt wird:

$$
G_{a}=G_{b}=G_{i}^{\otimes} \text {. }
$$

Setzt man Gleichung (4.36) in Gleichung (4.35) ein, so erhält man:

\footnotetext{
${ }^{\prime}$ Vgl. Heap /Varoufakis (1995, S. 55).
} 


$$
\frac{\left(\left.\frac{d E}{d G_{i}}\right|_{G_{i}=G_{i}^{\otimes}}\right)}{\left(\left.\frac{d C}{d G_{i}}\right|_{G_{i}=G_{i}^{\otimes}}\right)} \cdot f_{K a}\left(K_{a}\right)=\frac{\left(\left.\frac{d E}{d G_{i}}\right|_{G_{i}=G_{i}^{\otimes}}\right)}{\left(\left.\frac{d C}{d G_{i}}\right|_{G_{i}=G_{i}^{\otimes}}\right)} \cdot f_{K b}\left(K_{b}\right) .
$$

$\mathrm{Da}$ die Regionen vollkommen identisch sind, führt eine gleiche Menge an öffentlichem Input zu einer Gleichverteilung des Kapitals. Weil der Kapitalstock mit $\bar{K}$ exogen vorgegeben ist, entfällt auf jede Region somit eine Kapitalmenge in Höhe von $\frac{\bar{K}}{2}$ :

$$
K_{a}=K_{b}=\frac{\bar{K}}{2} .
$$

Gleichung (4.37) kann daher wie folgt geschrieben werden:

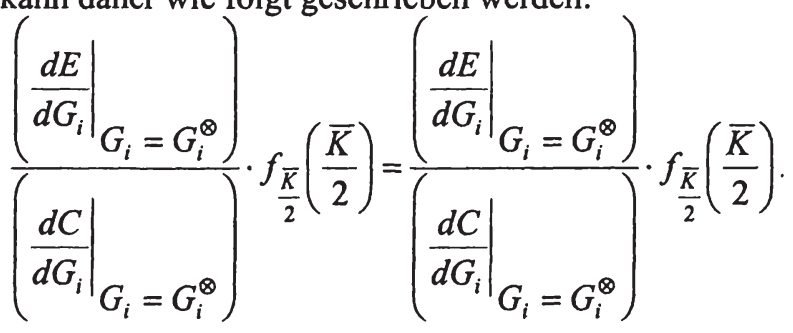

Da es sich bei Gleichung (4.39) um eine wahre Aussage handelt, konnte gezeigt werden, dass ein Nash-Gleichgewicht vorliegt, wenn beide Regierungen dieselbe, für sie optimale Menge an öffentlichem Input anbieten. Dieses NashGleichgewicht ist eindeutig. ${ }^{1}$ Die Höhe der gleichgewichtigen Menge $\left(G_{i}^{\otimes}\right)$ kann wahlweise aus Gleichung (4.33) oder Gleichung (4.34) ermittelt werden. Setzt man Gleichung (4.36) und Gleichung (4.38) ein, so ergibt sich:

${ }^{1}$ Für den Beweis, dass das Nash-Gleichgewicht eindeutig ist, siehe Anhang 10. 
$(4.40)$

$$
\frac{\left(\left.\frac{d E}{d G_{i}}\right|_{G_{i}=G_{i}^{\otimes}}\right)}{\left(\left.\frac{d C}{d G_{i}}\right|_{G_{i}=G_{i}^{\otimes}}\right) \cdot E\left(G_{i}^{\otimes}\right)}=\frac{(-2)}{\tilde{t}_{K}} \cdot \frac{f_{\bar{K} \bar{K}}\left(\frac{\bar{K}}{2}\right)}{f_{\frac{\bar{K}}{2}\left(\frac{\bar{K}}{2}\right)}} .
$$

Wie Gleichung (4.40) zu entnehmen ist, hängt die Höhe der für die Leviathanregierungen im Nash-Gleichgewicht optimalen Menge an öffentlichem Zwischenprodukt von den Technologien $\left(\Phi_{i}^{2}\right)$ und dem harmonisierten Steuersatz $\left(\tilde{t}_{K}\right)$ ab:

$$
G_{i}^{\otimes}=\Phi_{i}^{2}\left(\tilde{t}_{K}\right)
$$

\subsection{Die Bedeutung des harmonisierten Steuersatzes}

Bisher wurde also untersucht, wie sich Leviathanregierungen im Standortwettbewerb bei einem durch die Finanzverfassung exogen vorgegebenen, harmonisierten Kapitalsteuersatz verhalten. Diese Untersuchung stellt die Basis für die Beantwortung der Frage nach einer optimalen Finanzverfassung dar. Um dieser Frage nachzugehen, wird zunächst die Höhe des harmonisierten Steuersatzes variiert und dabei beobachtet, auf welche Weise der Steuersatz die Höhe der von den Regierungen im Nash-Gleichgewicht bereitgestellten Menge an öffentlichem Zwischenprodukt beeinflusst. Der Zusammenhang zwischen öffentlichem Input und Steuersatz lässt sich bestimmen, indem Gleichung (4.40) nach dem harmonisierten Steuersatz abgeleitet wird:'

${ }^{1} \mathrm{Vgl}$. für die Berechnung von Gleichung (4.42) Anhang 11. 


$$
\begin{aligned}
& \text { (4.42) } \quad \frac{d G_{i}^{\otimes}}{d \tilde{t}_{K}}=\left(\frac { | \frac { d E } { d G _ { i } } | _ { G _ { i } = G _ { i } ^ { \otimes } } ) } { ( \frac { d C } { d G _ { i } } | _ { G _ { i } = G _ { i } ^ { \otimes } } ) \cdot E ( G _ { i } ^ { \otimes } ) } \cdot \left(-\frac{\tilde{t}_{K} \cdot\left(\left.\frac{d^{2} E}{d G_{i}^{2}}\right|_{G_{i}=G_{i}^{\otimes}}\right)}{\left(\left.\frac{d C}{d G_{i}}\right|_{G_{i}=G_{i}^{\otimes}}\right) \cdot E\left(G_{i}^{\otimes}\right)}+\right.\right. \\
& \frac{\tilde{t}_{K} \cdot\left(\left.\frac{d E}{d G_{i}}\right|_{G_{i}=G_{i}^{\otimes}}\right) \cdot\left(\left.\frac{d^{2} C}{d G_{i}^{2}}\right|_{G_{i}=G_{i}^{\otimes}}\right)}{\left(\left.\frac{d C}{d G_{i}}\right|_{G_{i}=G_{i}^{\otimes}}\right)^{2} \cdot E\left(G_{i}^{\otimes}\right)}+\frac{\tilde{t}_{K} \cdot\left(\left.\frac{d E}{d G_{i}}\right|_{G_{i}=G_{i}^{\otimes}}\right)^{2}}{\left(\left.\frac{d C}{d G_{i}}\right|_{G_{i}=G_{i}^{\otimes}}\right) \cdot\left(E\left(G_{i}^{\otimes}\right)\right)^{2}}>0 .
\end{aligned}
$$

Die Menge an öffentlichem Zwischenprodukt ist also umso größer, je höher der Steuersatz ausfällt und umgekehrt (vgl. Abbildung 23).

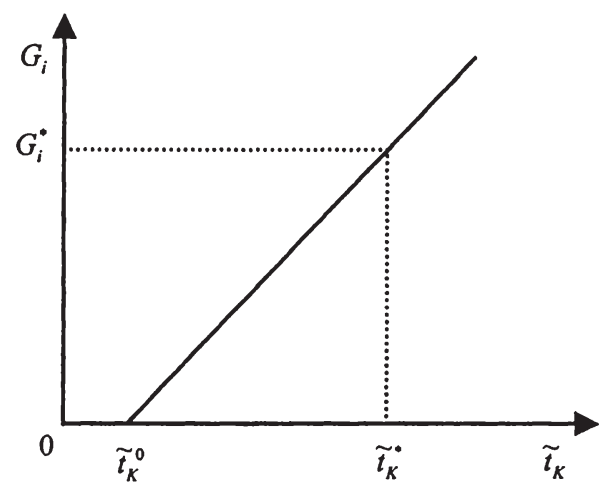

\section{Abbildung 23: Die Wirkung des harmonisierten Steuersatzes ${ }^{1}$}

Durch die verfassungsgemäß vorgeschriebene Höhe des harmonisierten Steuersatzes wird also letztlich indirekt die Menge an öffentlichem Input festgelegt. Der Leviathanstaat maximiert seinen Surplus, wenn die Grenzeinnahmen den

\footnotetext{
${ }^{1}$ Inwiefern der Zusammenhang zwischen dem Steuersatz und der Menge an öffentlichem Input überproportional, proportional oder unterproportional ist, kann nicht geklärt werden. Der Einfachheit halber wurde bei der Darstellung ein proportionaler Zusammenhang angenommen.
} 
Grenzkosten entsprechen. Die Höhe des Steuersatzes beeinflusst die Höhe der Einnahmen und somit auch die Höhe der Grenzeinnahmen. Je höher der harmonisierte Substanzsteuersatz ist, umso höher sind auch die Grenzeinnahmen je eingesetztem öffentlichen Input. Daher stimmen bei einem relativ hohen Steuersatz Grenzeinnahmen und Grenzkosten erst bei einer größeren Menge an öffentlichem Zwischenprodukt überein als bei einem niedrigen Steuersatz.

Durch den in der Verfassung festgelegten, harmonisierten Steuersatz kann nun verhindert werden, dass die Regierungen überhaupt einen Anreiz haben, das öffentliche Zwischenprodukt bereitzustellen. Dies ist der Fall, wenn der Steuersatz $\left(\tilde{t}_{K}\right)$ einem bestimmten kritischen Wert $\left(\tilde{t}_{K}^{0}\right)$ entspricht oder ihn gar unterschreitet (vgl. Abbildung 23):

$$
\tilde{t}_{K} \leq \tilde{t}_{K}^{0} \text {. }
$$

Für den Fall, dass der Steuersatz genau mit dem kritischen Wert übereinstimmt, liegt das globale Surplusmaximum bei $G_{i}^{\otimes}=0$. Es handelt sich also um den in Kapitel 4.2 bereits diskutierten Fall 2, bei dem die Regierungen ihren Surplus maximieren, indem sie das öffentliche Zwischenprodukt nicht anbieten. Liegt der Steuersatz unterhalb dieses kritischen Werts, so besteht ein lokales Maximum bei $G_{i}^{\otimes}=0$. In diesem in Kapitel 4.2 als Fall 1 behandelten Fall stellen die Regierungen das öffentliche Zwischenprodukt ebenfalls nicht bereit. Berücksichtigt man Gleichung (4.40), so zeigt sich, dass dieser kritische Steuersatz

$$
\tilde{t}_{K}^{0}=(-2) \cdot \frac{\left(\left.\frac{d C}{d G_{i}}\right|_{G_{i}=0}\right) \cdot E(0)}{\left(\left.\frac{d E}{d G_{i}}\right|_{G_{i}=0}\right)} \cdot \frac{f_{\frac{\bar{K}}{2} \frac{K}{2}}\left(\frac{\bar{K}}{2}\right)}{f_{\frac{\bar{K}}{2}}\left(\frac{\bar{K}}{2}\right)}>0
$$

entspricht. Ob die Regierungen das öffentliche Zwischenprodukt überhaupt bereitstellen, wird also durch die Finanzverfassung bereits festgelegt. Damit die Leviathanstaaten einen eigenständigen finanziellen Anreiz haben, das öffentliche Zwischenprodukt anzubieten, muss der harmonisierte Steuersatz größer als der kritische Steuersatz sein:

$$
\tilde{t}_{K}>\tilde{t}_{K}^{0} \text {. }
$$


Fraglich ist dann jedoch immer noch, ob das öffentliche Zwischenprodukt effizient bereitgestellt wird. Nach der Kaizuka-Regel ${ }^{1}$ wird das öffentliche Zwischenprodukt effizient angeboten, wenn die Menge des öffentlichen Inputs so gewählt wird, dass die Grenzkosten der Produktion des öffentlichen Zwischenprodukts gerade mit dem Wertgrenzertrag aus dem Einsatz des öffentlichen Zwischenprodukts übereinstimmen: ${ }^{2}$

$$
\left(\left.\frac{d E}{d G_{i}}\right|_{G_{i}=G_{i}^{\otimes}}\right) \cdot f_{i}\left(\frac{\bar{K}}{2}, \bar{L}_{i}\right)=\left(\left.\frac{d C}{d G_{i}}\right|_{G_{i}=G_{i}^{\otimes}}\right) .
$$

Betrachtet man Gleichung (4.40), so zeigt sich, dass die Regierungen freiwillig so viel öffentliches Gut bereitstellen, dass

$$
\left(\left.\frac{d E}{d G_{i}}\right|_{G_{i}=G_{i}^{\otimes}}\right) \cdot \frac{\tilde{t}_{K}}{(-2) \cdot E\left(G_{i}^{\otimes}\right)} \cdot \frac{f_{\bar{K}}\left(\frac{\bar{K}}{2}\right)}{f_{\frac{\bar{K}}{2} \frac{\bar{K}}{2}}\left(\frac{\bar{K}}{2}\right)}=\left(\left.\frac{d C}{d G_{i}}\right|_{G_{i}=G_{i}^{\otimes}}\right)
$$

gültig ist. Möchte man nun garantieren, dass die Regierungen freiwillig eine effiziente Menge an öffentlichem Input $\left(G_{i}^{*}\right)$ anbieten, dann ist der harmonisierte Steuersatz $\left(\tilde{t}_{K}^{*}\right)$ von der Verfassung so vorzugeben, dass

$$
\frac{\tilde{t}_{K}^{*}}{(-2) \cdot E\left(G_{i}^{*}\right)} \cdot \frac{f_{\bar{K}}\left(\frac{\bar{K}}{2}\right)}{f_{\frac{\bar{K}}{2} \bar{K} \frac{\bar{K}}{2}}\left(\frac{\bar{K}}{2}\right)}=f_{i}\left(\frac{\bar{K}}{2}, \bar{L}_{i}\right)
$$

vorliegt. Im Nash-Gleichgewicht bieten daher die Regierungen eine effiziente Menge an öffentlichem Input an, wenn der harmonisierte Steuersatz genau

${ }^{1}$ Diese Regel wurde von Kaizuka (1965) in Anlehnung an die Samuelsonbedingung aufgestellt. Während die Samuelsonbedingung eine Bedingung für den effizienten Einsatz von öffentlichen Konsumgütern darstellt, beschreibt die Kaizuka-Regel die Bedingung für die produktionseffiziente Bereitstellung von offentlichen Inputs. Vgl. Samuelson (1954), Feehan (1989, S. 238), Colombier / Pickhardt (1999, S. 27 ff.), Colombier (2001, S. 28).

${ }^{2}$ Vgl. Colombier (2001, S. 32). 


$$
\left.\tilde{t}_{K}^{*}=E\left(G_{i}^{*}\right) \cdot f_{i}\left(\frac{\bar{K}}{2}, \bar{L}_{i}\right)^{(-2) \cdot f_{\frac{\bar{K}}{2} \frac{\bar{K}}{2}}\left(\frac{\bar{K}}{2}\right)} f_{\frac{\bar{K}}{2}\left(\frac{\bar{K}}{2}\right)}\right) 0
$$

entspricht (siehe Abbildung 23). Liegt der harmonisierte Steuersatz nämlich bei $\tilde{t}_{K}^{*}$, so können beide Leviathanregierungen ihren Surplus nur maximieren, wenn sie das öffentliche Zwischenprodukt effizient bereitstellen. Wie Gleichung (4.49) zu entnehmen ist, wird die Höhe des harmonisierten Steuersatzes, die zu einer effizienten Bereitstellung des öffentlichen Inputs führt, letztlich durch die Technologien zur Produktion des privaten Gutes determiniert.

\subsection{Zusammenfassung}

Es hat sich somit gezeigt, dass die Regierungen auch bei einer Harmonisierung der Steuern in einer Föderation einen Wettbewerb um Kapital eingehen. Bei diesem Wettbewerb handelt es sich nicht um einen Steuerwettbewerb, weil der aufgrund der Steuerharmonisierung unterbunden wird, sondern um einen Ausgabenwettbewerb. In diesem Ausgabenwettbewerb versuchen die einzelnen Regierungen, über ein unterschiedliches Angebot an öffentlichen Zwischenprodukten Kapital in ihre Region zu locken. Dadurch kann dann für die einzelnen Regierungen ein eigener finanzieller Anreiz entstehen, das öffentliche Zwischenprodukt anzubieten.

Des Weiteren konnte dargelegt werden, dass die Höhe des durch die Finanzverfassung vorgegebenen, harmonisierten Steuersatzes von fundamentaler Bedeutung für den Ausgabenwettbewerb ist. Wird der Steuersatz zu niedrig angesetzt, so entsteht dadurch für die Regierungen der Anreiz, die Regionen mit öffentlichen Zwischenprodukten unterzuversorgen. Eine solche Finanzverfassung ist somit weder im Interesse der Bürger, da die Versorgung mit öffentlichen Zwischenprodukten suboptimal ist, noch im Interesse der Leviathanstaaten, da deren Einnahmen stark begrenzt werden. Erst ein entsprechend hoher Steuersatz auf das Kapital $\left(\tilde{t}_{K}^{*}\right)$ bietet dem Leviathan im Standortwettbewerb genügend Anreiz, um das öffentliche Zwischenprodukt in effizientem Umfang bereitzustellen. Schreibt die Finanzverfassung also den Kapitalsteuersatz $\tilde{t}_{\kappa}^{*}$ vor, so ist die- 
se Finanzverfassung für die Bürger insofern optimal, als sie ein effizientes Angebot an öffentlichem Zwischenprodukt sicherstellt und die Besteuerungsmacht des Leviathans im notwendigen Maße einschränkt.

\section{Fazit und Ausblick}

In dieser Arbeit wurde die Bedeutung von Ausgaben für öffentliche Zwischenprodukte im zwischenstaatlichen Wettbewerb um Kapital herausgearbeitet. Es wurde gezeigt, dass die fiskalpolitischen Instrumente - Steuern und Ausgaben für öffentliche Zwischenprodukte - als strategische Variablen zur Attrahierung von Kapital substituierbar sind. Untersuchungen zum fiskalischen Standortwettbewerb, die sich ausschließlich auf den Steuerwettbewerb beziehen, greifen somit zu kurz. Steuersatzsenkungen in einer Region führen nämlich nicht notwendigerweise zu einem höheren fiskalpolitischen Instrumenten-Index in dieser Region. Bei einer gleichzeitig zur Finanzierung der Steuersenkung vorgenommenen Ausgabensenkung ist insbesondere bei einem relativ niedrigen Ausgangsniveau des Steuersatzes zu erwarten, dass der fiskalpolitische Instrumenten-Index sinkt. C.p. bewirkt dann diese Kombination von Steuersatz- und Ausgabensenkung, dass die Menge an attrahiertem Kapital in der betreffenden Region sinkt. Untersuchungen, die ausschließlich den Steuerwettbewerb analysieren, würden jedoch bei einer Steuersatzsenkung einer Region generell eine Erhöhung der Menge an Kapital in dieser Region vorhersagen, da die Wirkung des öffentlichen Zwischenproduktes modelltheoretisch nicht erfasst wird und somit unbeachtet bleibt. Diese konträren Ergebnisse veranschaulichen die Wichtigkeit, öffentliche Zwischenprodukte im Modell zu integrieren und insbesondere ihre Wirkung auf das Kapital explizit im Modell einzubeziehen.

Unter diesem Aspekt ist dann auch die Vorstellung unhaltbar, dass die Harmonisierung von Steuern den fiskalischen Wettbewerb der Regionen unterbinden könnte. Durch eine Harmonisierung der Steuern in einer Föderation wird lediglich Steuerwettbewerb verhindert. Da der Steuerwettbewerb jedoch nur einen Teil des fiskalischen Wettbewerbs darstellt, konkurrieren die Regionen im Rahmen des Ausgabenwettbewerbs weiterhin miteinander um Kapital. Allerdings wird dieser Ausgabenwettbewerb nicht unmaßgeblich von den durch die Verfassung festgesetzten Regeln zur Harmonisierung der Steuern beeinflusst. Liegt 
der harmonisierte Steuersatz zu niedrig, so werden die Regierungen eine zu geringe Menge an öffentlichem Zwischenprodukt bereitstellen. Es kommt zu einer Unterversorgung mit öffentlichen Zwischenprodukten. Erst ein entsprechend hoher Steuersatz $\left(\tilde{t}_{k}^{*}\right)$ gibt den Regierungen den Anreiz, dass sie das öffentliche Zwischenprodukt in effizientem Umfang anbieten. Generell ist ein positiver $\mathrm{Zu}$ sammenhang zwischen der Höhe des Steuersatzes und dem aus dem zwischenstaatlichen Wettbewerb resultierenden Angebot an öffentlichem Input festzustellen. Indirekt beeinflussen somit die Verfassungsregeln zur Steuerharmonisierung das Ergebnis des Ausgabenwettbewerbs.

Diese Resultate wurden mit Hilfe eines Kapitalmarktmodells erhalten, dass einen exogen vorgegebenen Kapitalbestand annimmt. Änderungen der Fiskalpolitik führen daher zu einer Anpassung des Nettozinssatzes und zu einer Kapitalrelokation zwischen den Regionen. Da eine Region Kapital von den anderen Regionen abziehen muss, um selbst zusätzliches Kapital zu erhalten, ist dieses Kapitalmarktmodell in der Lage, fiskalischen Wettbewerb um Kapital darzustellen. Durch die Verwendung dieses Modells wurde deutlich, dass bei einer Steuersatzsenkung einer Region nicht nur durch die gleichzeitig notwendige Ausgabenreduktion eine Attrahierung von zusätzlichem Kapital verhindert werden kann. Ebenso kann durch die Fiskalpolitik der Konkurrenzregionen selbst bei steigendem fiskalpolitischen Instrumenten-Index Kapital aus der eigenen Region abgezogen werden. Dieser Effekt würde bei einem Modell, dass von kleinen Regionen mit vollkommen preiselastischem Kapitalangebot ausgeht, nicht sichtbar werden. Daher ist das Modell mit vollkommen preiselastischem Kapitalangebot auch nicht geeignet, um fiskalischen Wettbewerb um Kapital abzubilden. Es ist jedoch zu bedenken, dass die Ergebnisse der Arbeit zumindest in Teilen auf der Annahme einer Leviathanregierung basieren. Diese Annahme wurde als erste Näherung für eine eigennützige Regierung verwendet, um die Untersuchung alleinig auf die unmittelbare Wirkung des fiskalischen Wettbewerbs auf eigennützige Regierungen zu konzentrieren. Durch sie war ein praktikabler Einstieg in die Untersuchung des Ausgabenwettbewerbs zwischen eigennützigen Regierungen möglich. Weitaus realistischer wäre hingegen die Verwendung eines Modells mit einer eigennützigen Regierung, wie sie Edwards und Keen vorschlagen, gewesen. Eine entsprechende Erweiterung des Modells wird in einer späteren Untersuchung angestrebt. 
Darüber hinaus wurden die Untersuchungen auf die Analyse der allokativen Wirkung von fiskalischem Standortwettbewerb beschränkt. Allerdings weisen die in Kapitel 3 Abschnitt 4.3 erhaltenen Ergebnisse auf die Bedeutung der distributiven Wirkung von fiskalischem Wettbewerb hin. Dort hatte sich gezeigt, dass eine ruinöse Wettbewerbsspirale in Gang gesetzt wird, wenn beide Regierungen gleichzeitig das Ziel verfolgen, zusätzliches Kapital zu attrahieren. Dabei bleibt die Kapitallokation unverändert, wobei der Nettozinssatz stetig ansteigt. Die intendierte allokative Wirkung unterbleibt somit. Demgegenüber führt der Wettbewerb zwischen den Regierungen unter Umständen eine von den Regierungen nicht intendierte distributive Wirkung mit sich. Diese im Rahmen dieser Untersuchung lediglich angerissene Problematik der Änderung der Einkommensdistribution durch fiskalischen Wettbewerb bedarf ebenfalls tiefgehenderer Untersuchungen. Insbesondere wäre die unterschiedliche distributive Wirkung von Steuer- und Ausgabenwettbewerb herauszuarbeiten. 


\section{Anhang 1}

\section{Abschnitt a}

Gleichung (2.38) stellt eine implizite Funktion des Nettozinssatzes (r) dar:

$\bar{K}=f_{z a}\left(r+t_{a, s}\right)+f_{z b}\left(r+t_{b, s}\right)$.

Leitet man Gleichung (2.38) nach $t_{a, s} a b$, so ergibt sich:

$$
\begin{aligned}
& \frac{\partial \bar{K}}{\partial t_{a, s}}=f_{z a z a}\left(r+t_{a, s}\right) \cdot \frac{\partial\left(r+t_{a, s}\right)}{\partial t_{a, s}}+f_{z b z b}\left(r+t_{b, s}\right) \cdot \frac{\partial\left(r+t_{b, s}\right)}{\partial t_{a, s}} \\
& \Leftrightarrow \quad 0=f_{z a z a}\left(r+t_{a, s}\right) \cdot\left(\frac{\partial r}{\partial t_{a, s}}+1\right)+f_{z b z b}\left(r+t_{b, s}\right) \cdot \frac{\partial r}{\partial t_{a, s}} \\
& \Leftrightarrow \quad 0=f_{z a z a}\left(r+t_{a, s}\right)+f_{z a z a}\left(r+t_{a, s}\right) \cdot \frac{\partial r}{\partial t_{a, s}}+f_{z b z b}\left(r+t_{b, s}\right) \cdot \frac{\partial r}{\partial t_{a, s}} \\
& \Leftrightarrow \quad-f_{z a z a}\left(r+t_{a, s}\right)=\left(f_{z a z a}\left(r+t_{a, s}\right)+f_{z b z b}\left(r+t_{b, s}\right)\right) \cdot \frac{\partial r}{\partial t_{a, s}} \\
& \Leftrightarrow \quad \frac{\partial r}{\partial t_{a, s}}=-\frac{f_{z a z a}\left(r+t_{a, s}\right)}{\left(f_{z a z a}\left(r+t_{a, s}\right)+f_{z b z b}\left(r+t_{b, s}\right)\right)}
\end{aligned}
$$

Die Ableitung des Nettozinssatzes nach dem Substanzsteuersatz $\left(t_{a, s}\right)$ entspricht also:

$$
\frac{\partial r}{\partial t_{a, s}}=\frac{-1}{\left(1+\frac{f_{z b z b}\left(r+t_{b, s}\right)}{f_{z a z a}\left(r+t_{a, s}\right)}\right)} \text {. }
$$

\section{Abschnitt b}

Gleichung (2.39) ist eine implizite Funktion des Nettozinssatzes (r):

$$
\bar{K}=f_{z a}\left(\frac{r}{\left(1-t_{a, e}\right)}\right)+f_{z b}\left(\frac{r}{\left(1-t_{b, e}\right)}\right) \text {. }
$$

Durch Ableitung der Funktion nach $\mathrm{t}_{\mathrm{a}, \mathrm{e}}$ und Umformung gelangt man zu: 
88

$$
\begin{aligned}
& \frac{\partial \bar{K}}{\partial t_{a, e}}=f_{z a z a}\left(\frac{r}{\left(1-t_{a, e}\right)}\right) \cdot \frac{\partial\left(\frac{r}{\left(1-t_{a, e}\right)}\right)}{\partial t_{a, e}}+f_{z b z b}\left(\frac{r}{\left(1-t_{b, e}\right)}\right) \cdot \frac{\partial\left(\frac{r}{\left(1-t_{b, e}\right)}\right)}{\partial t_{a, e}} \\
& \Leftrightarrow 0=f_{z a z a}\left(\frac{r}{\left(1-t_{a, e}\right)}\right) \cdot\left(\frac{\partial r}{\partial t_{a, e}} \cdot \frac{1}{\left(1-t_{a, e}\right)}+\frac{r}{\left(1-t_{a, e}\right)^{2}}\right) \\
& +f_{z b z b}\left(\frac{r}{\left(1-t_{b, e}\right)}\right) \cdot\left(\frac{\partial r}{\partial t_{a, e}} \cdot \frac{1}{\left(1-t_{b, e}\right)}\right) \\
& \Leftrightarrow 0=\frac{f_{z a z a}\left(\frac{r}{\left(1-t_{a, e}\right)}\right)}{\left(1-t_{a, e}\right)} \cdot \frac{\partial r}{\partial t_{a, e}}+\frac{f_{z a z a}\left(\frac{r}{\left(1-t_{a, e}\right)}\right)}{\left(1-t_{a, e}\right)} \cdot \frac{r}{\left(1-t_{a, e}\right)} \\
& +\frac{f_{z b z b}\left(\frac{r}{\left(1-t_{b, e}\right)}\right)}{\left(1-t_{b, e}\right)} \cdot \frac{\partial r}{\partial t_{a, e}} \\
& \Leftrightarrow\left(\frac{f_{z a z a}\left(\frac{r}{\left(1-t_{a, e}\right)}\right)}{\left(1-t_{a, e}\right)}+\frac{f_{z b z b}\left(\frac{r}{\left(1-t_{b, e}\right)}\right)}{\left(1-t_{b, e}\right)}\right) \cdot \frac{\partial r}{\partial t_{a, e}}=\frac{-r}{\left(1-t_{a, e}\right)} \cdot \frac{f_{z a z a}\left(\frac{r}{\left(1-t_{a, e}\right)}\right)}{\left(1-t_{a, e}\right)}
\end{aligned}
$$

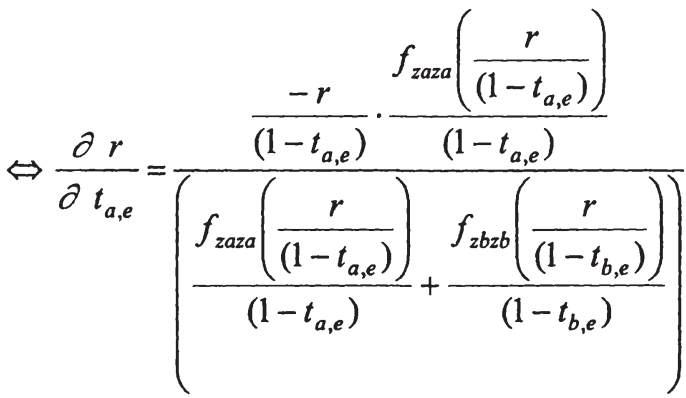

Claudia Hensberg - 978-3-631-75213-5

Downloaded from PubFactory at 01/11/2019 07:02:00AM

via free access 


$$
\begin{aligned}
& \Leftrightarrow \frac{\partial r}{\partial t_{a, e}}=\frac{-1}{\left(1-t_{a, e}\right)} \cdot \frac{\frac{f_{z a z a}\left(\frac{r}{\left(1-t_{a, e}\right)}\right)}{\left(1-t_{a, e}\right)}}{\left(\frac{f_{z a z a}\left(\frac{r}{\left(1-t_{a, e}\right)}\right)}{\partial t_{a, e}}+\frac{f_{z b z b}\left(\frac{r}{\left(1-t_{a, e}\right)}\right)}{\left(1-t_{b, e}\right)}\right)} \\
& \left.\Leftrightarrow \frac{-1}{\left(1-t_{a, e}\right)} \cdot \frac{\frac{r}{f_{z b z b}\left(\frac{r}{\left(1-t_{b, e}\right)}\right)}}{\left(1-t_{b, e}\right)}\right) \\
& \left.\frac{f_{z a z a}\left(\frac{r}{\left(1-t_{a, e}\right)}\right)}{\left(1-t_{a, e}\right)}\right)
\end{aligned}
$$

Als Ergebnis erhält man somit:

$$
\frac{\partial r}{\partial t_{a, e}}=\frac{-1}{\left(1-t_{a, e}\right)} \cdot \frac{r}{\left.1+\frac{\left(1-t_{a, e}\right) \cdot f_{z b z b}\left(\frac{r}{\left(1-t_{b, e}\right)}\right)}{\left(1-t_{b, e}\right) \cdot f_{z a z a}\left(\frac{r}{\left(1-t_{a, e}\right)}\right)}\right)} \text {. }
$$




\section{Anhang 2}

\section{Abschnitt a}

Nach Gleichung (2.45) und Gleichung (2.35) kommt folgende Kapitalmenge in Region a zum Einsatz:

$K_{a}=f_{z a}\left(r+t_{a, s}\right)$.

Durch Ableitung dieser Gleichung nach $\mathrm{t}_{\mathrm{a}, \mathrm{s}}$ resultiert:

$\frac{\partial K_{a}}{\partial t_{a, s}}=f_{z a z a}\left(r+t_{a, s}\right) \cdot\left(\frac{\partial r}{\partial t_{a, s}}+1\right)$.

Ersetzt man nun $\frac{\partial r}{\partial t_{a, s}}$ durch $\frac{-1}{\left(1+\frac{f_{z b z b}\left(r+t_{b, s}\right)}{f_{z a z a}\left(r+t_{a, s}\right)}\right)}$

(vgl. Gleichung (2.40)) und

formt um, dann folgt daraus:

$\frac{\partial K_{a}}{\partial t_{a, s}}=f_{z a z a}\left(r+t_{a, s}\right) \cdot\left(\frac{-1}{\left(1+\frac{f_{z b z b}\left(r+t_{b, s}\right)}{f_{z a z a}\left(r+t_{a, s}\right)}\right)}+1\right)$

$\Leftrightarrow \frac{\partial K_{a}}{\partial t_{a, s}}=f_{z a z a}\left(r+t_{a, s}\right) \cdot\left(\frac{-1+\left(1+\frac{f_{z b z b}\left(r+t_{b, s}\right)}{f_{z a z a}\left(r+t_{a, s}\right)}\right)}{\left(1+\frac{f_{z b z b}\left(r+t_{b, s}\right)}{f_{z a z a}\left(r+t_{a, s}\right)}\right)}\right)$

$\left.\Leftrightarrow \frac{\partial K_{a}}{\partial t_{a, s}}=f_{z a z a}\left(r+t_{a, s}\right) \cdot\left(\frac{\frac{f_{z b z b}\left(r+t_{b, s}\right)}{f_{z a z a}\left(r+t_{a, s}\right)}}{\left(1+\frac{f_{z b z b}\left(r+t_{b, s}\right)}{f_{z a z a}\left(r+t_{a, s}\right.}\right)}\right)\right)$

$\left.\Leftrightarrow \frac{\partial K_{a}}{\partial t_{a, s}}=\left(\frac{f_{z b z b}\left(r+t_{b, s}\right)}{\left(1+\frac{f_{z b z b}\left(r+t_{b, s}\right.}{f_{z a z a}\left(r+t_{a, s}\right.}\right)}\right)\right)$ 
$\Leftrightarrow \frac{\partial K_{a}}{\partial t_{a, s}}=\left(\frac{f_{z b z b}\left(r+t_{b, s}\right)}{f_{z b z b}\left(r+t_{b, s}\right) \cdot\left(\frac{1}{f_{z b z b}\left(r+t_{b, s}\right)}+\frac{1}{f_{z a z a}\left(r+t_{a, s}\right)}\right)}\right)$.

Man gelangt dadurch zu folgendem Resultat:

$$
\frac{\partial K_{a}}{\partial t_{a, s}}=\left(\frac{1}{\left(\frac{1}{f_{z b z b}\left(r+t_{b, s}\right)}+\frac{1}{f_{z a z a}\left(r+t_{a, s}\right)}\right)}\right) \text {. }
$$

\section{Abschnitt b}

Nach Gleichung (2.47) und Gleichung (2.35) wird folgende Kapitalmenge in Region a eingesetzt:

$$
K_{a}=f_{z a}\left(\frac{r}{\left(1-t_{a, e}\right)}\right) \text {. }
$$

Durch Ableitung dieser Gleichung nach $\mathrm{t}_{\mathrm{a}, \mathrm{e}}$ erhält man:

$$
\frac{\partial K_{a}}{\partial t_{a, e}}=f_{z a z a}\left(\frac{r}{\left(1-t_{a, e}\right)}\right) \cdot\left(\frac{\partial r}{\partial t_{a, e}} \cdot \frac{1}{\left(1-t_{a, e}\right)}+\frac{r}{\left(1-t_{a, e}\right)^{2}}\right) \text {. }
$$

Ersetzt man nun $\frac{\partial r}{\partial t_{a, e}}$ durch $\frac{-1}{\left(1-t_{a, e}\right)} \cdot \frac{r}{\left(1+\frac{\left(1-t_{a, e}\right) \cdot f_{z b z b}\left(\frac{r}{\left(1-t_{b, e}\right)}\right)}{\left(1-t_{b, e}\right) \cdot f_{z a z a}\left(\frac{r}{\left(1-t_{a, e}\right)}\right)}\right)}$ (siehe

Gleichung (2.42)) und formt um, dann ergibt sich: 


$$
\begin{aligned}
& \frac{\partial K_{a}}{\partial t_{a, e}}=\frac{f_{z a z a}\left(\frac{r}{\left(1-t_{a, e}\right)}\right)}{\left(1-t_{a, e}\right)} \cdot\left(\frac{-1}{\left(1-t_{a, e}\right)} \cdot \frac{r}{\left(1+\frac{\left(1-t_{a, e}\right) \cdot f_{z b z b}\left(\frac{r}{\left(1-t_{b, e}\right)}\right)}{\left(1-t_{b, e}\right) \cdot f_{z a z a}\left(\frac{r}{\left(1-t_{a, e}\right)}\right)}+\frac{r}{\left(1-t_{a, e}\right)}\right)}\right. \\
& \Leftrightarrow \frac{\partial K_{a}}{\partial t_{a, e}}=\frac{f_{z a z a}\left(\frac{r}{\left(1-t_{a, e}\right)}\right)}{\left(1-t_{a, e}\right)} \cdot \frac{r}{\left(1-t_{a, e}\right)}\left(\frac{-1}{\left(1-\frac{\left(1-t_{a, e}\right) \cdot f_{z b z b}\left(\frac{r}{\left(1-t_{b, e}\right)}\right)}{\left(1-t_{b, e}\right) \cdot f_{z a z a}\left(\frac{r}{\left(1-t_{a, e}\right)}\right)}\right)}\right)
\end{aligned}
$$

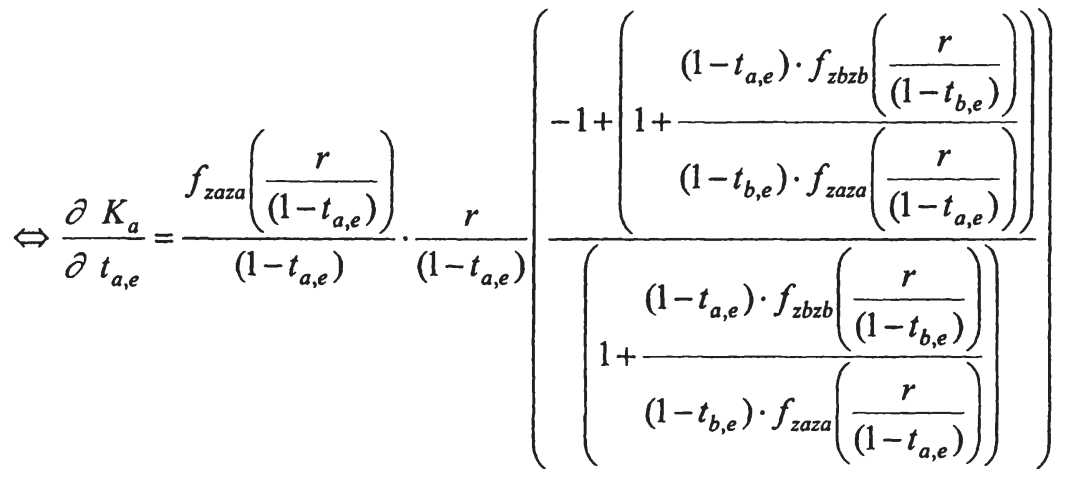




$$
\begin{aligned}
& \Leftrightarrow \frac{\partial K_{a}}{\partial t_{a, e}}=\frac{f_{z a z a}\left(\frac{r}{\left(1-t_{a, e}\right)}\right)}{\left(1-t_{a, e}\right)} \cdot \frac{r}{\left(1-t_{a, e}\right)}\left(\frac{\left(1-t_{a, e}\right) \cdot f_{z b z b}\left(\frac{r}{\left(1-t_{b, e}\right)}\right)}{\left(1-t_{b, e}\right) \cdot f_{z a z a}\left(\frac{r}{\left(1-t_{a, e}\right)}\right)}\right) \\
& \Leftrightarrow \frac{\partial K_{a}}{\partial t_{a, e}}=\frac{r}{\left(1-t_{a, e}\right)} \cdot\left(\frac{f_{z b z b}\left(\frac{r}{\left(1-t_{b, e}\right)}\right)}{\left(1-t_{b, e}\right)}\left(\frac{\left(1-t_{a, e}\right) \cdot f_{z b z b}\left(\frac{r}{\left(1-t_{b, e}\right)}\right)}{\left(1+\frac{r}{\left(1-t_{b, e}\right) \cdot f_{z a z a}\left(\frac{r}{\left(1-t_{a, e}\right)}\right)}\right)}\right)\right.
\end{aligned}
$$

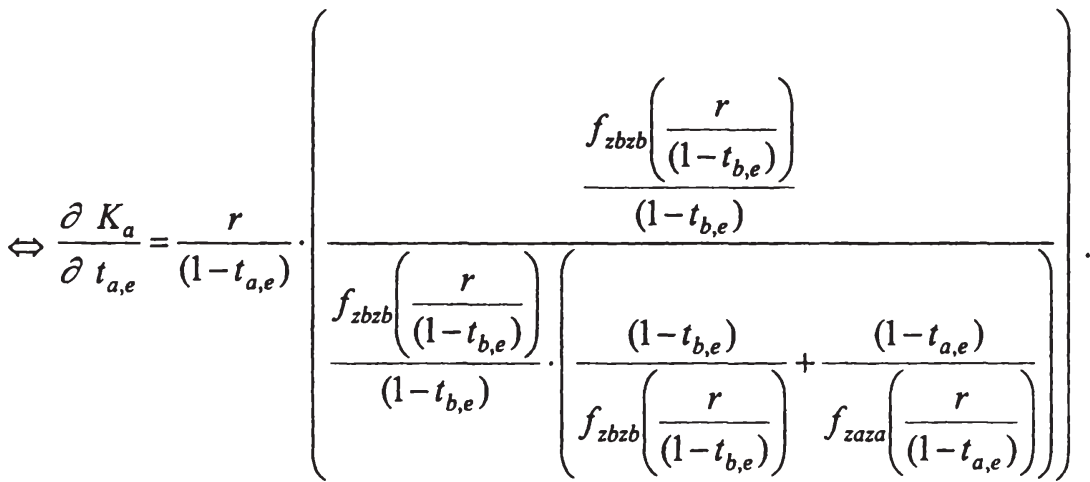

Die Ableitung des Kapitals $\left(\mathrm{K}_{\mathrm{a}}\right)$ nach dem Kapitalertragsteuersatz $\left(\mathrm{t}_{\mathrm{a}, \mathrm{e}}\right)$ stellt sich demnach dar als: 
$\frac{\partial K_{a}}{\partial t_{a, e}}=\frac{r}{\left(1-t_{a, e}\right)} \cdot\left(\frac{1}{\left(\frac{\left(1-t_{b, e}\right)}{f_{z b z b}\left(\frac{r}{\left(1-t_{b, e}\right)}\right)}+\frac{\left(1-t_{a, e}\right)}{f_{z a z a}\left(\frac{r}{\left(1-t_{a, e}\right)}\right)}\right)}\right)$. 


\section{Anhang 3}

\section{Abschnitt a}

Nach Gleichung (2.45) und Gleichung (2.35) entspricht die Kapitalmenge in Region a:

$K_{a}=f_{z a}\left(r+t_{a, s}\right)$.

Durch Ableitung dieser Gleichung nach $t_{b, s}$ folgt:

$\frac{\partial K_{a}}{\partial t_{b, s}}=f_{z a z a}\left(r+t_{a, s}\right) \cdot\left(\frac{\partial r}{\partial t_{b, s}}\right)$.

Ersetzt man nun $\frac{\partial r}{\partial t_{b, s}}$ durch $\frac{-1}{\left(1+\frac{f_{z a z a}\left(r+t_{a, s}\right)}{f_{z b z b}\left(r+t_{b, s}\right)}\right)}$ (vgl. Gleichung (2.41)) und

formt um, dann resultiert daraus:

$\frac{\partial K_{a}}{\partial t_{b, s}}=f_{z a z a}\left(r+t_{a, s}\right) \cdot\left(\frac{-1}{\left(1+\frac{f_{z a z a}\left(r+t_{a, s}\right)}{f_{z b z b}\left(r+t_{b, s}\right)}\right)}\right)$

$\Leftrightarrow \frac{\partial K_{a}}{\partial t_{b, s}}=\left(\frac{-f_{z a z a}\left(r+t_{a, s}\right)}{f_{z a z a}\left(r+t_{a, s}\right) \cdot\left(\frac{1}{f_{z a z a}\left(r+t_{a, s}\right)}+\frac{1}{f_{z b z b}\left(r+t_{b, s}\right)}\right)}\right)$

$\Leftrightarrow \frac{\partial K_{a}}{\partial t_{b, s}}=\left(\frac{-1}{\left(\frac{1}{f_{z b z b}\left(r+t_{b, s}\right)}+\frac{1}{f_{z a z a}\left(r+t_{a, s}\right)}\right)}\right)$. 
Da nach Gleichung (2.51) $\frac{\partial K_{b}}{\partial t_{b, s}}=\left(\frac{1}{\left(\frac{1}{f_{z b z b}\left(r+t_{b, s}\right)}+\frac{1}{f_{z a z a}\left(r+t_{a, s}\right)}\right)}\right)$ vorliegt, ist somit $-\frac{\partial K_{b}}{\partial t_{b, s}}=\frac{\partial K_{a}}{\partial t_{b, s}}$ gegeben.

\section{Abschnitt b}

Nach Gleichung (2.47) und Gleichung (2.35) wird in Region a soviel Kapital eingesetzt, dass gilt:

$K_{a}=f_{z a}\left(\frac{r}{\left(1-t_{a, e}\right)}\right)$.

Durch Ableitung dieser Gleichung nach $\mathrm{t}_{\mathrm{b}, \mathrm{e}}$ erhält man:

$\frac{\partial K_{a}}{\partial t_{b, e}}=f_{z a z a}\left(\frac{r}{\left(1-t_{a, e}\right)}\right) \cdot\left(\frac{\partial r}{\partial t_{b, e}} \cdot \frac{1}{\left(1-t_{a, e}\right)}\right)$.

Ersetzt man nun $\frac{\partial r}{\partial t_{b, e}}$ durch $\frac{-1}{\left(1-t_{b, e}\right)} \cdot \frac{r}{\left(1+\frac{\left(1-t_{b, e}\right) \cdot f_{z a z a}\left(\frac{r}{\left(1-t_{a, e}\right)}\right)}{\left(1-t_{a, e}\right) \cdot f_{z b z b}\left(\frac{r}{\left(1-t_{b, e}\right)}\right)}\right)}$ (siehe

Gleichung (2.43)) und formt um, dann folgt dadurch:

$$
\frac{\partial K_{a}}{\partial t_{b, e}}=\frac{f_{z a z a}\left(\frac{r}{\left(1-t_{a, e}\right)}\right)}{\left(1-t_{a, e}\right)} \cdot \frac{-1}{\left(1-t_{b, e}\right)} \cdot \frac{r}{\left(1+\frac{\left(1-t_{b, e}\right) \cdot f_{z a z a}\left(\frac{r}{\left(1-t_{a, e}\right)}\right)}{\left(1-t_{a, e}\right) \cdot f_{z b z b}\left(\frac{r}{\left(1-t_{b, e}\right)}\right)}\right)}
$$


97

$$
\begin{aligned}
& \Leftrightarrow \frac{\partial K_{a}}{\partial t_{b}}=\frac{-1}{\left(1-t_{b}\right)} \cdot \frac{\frac{f_{\text {zaza }}\left(\frac{r}{\left(1-t_{a, e}\right)}\right)}{\left(1-t_{a, e}\right)} \cdot r}{(-2)} \\
& \Leftrightarrow \frac{\partial K_{a}}{\partial t_{b, e}}=\frac{-1}{\left(1-t_{b, e}\right)} \cdot \frac{\frac{\left(1-t_{a, e}\right)}{f_{z a z a}\left(\frac{r}{\left(1-t_{a, e}\right)}\right)}}{\left.\frac{\left(1-t_{a, e}\right)}{\left(f_{z a z a}\left(\frac{r}{\left(1-t_{a, e}\right)}\right)\right.}+\frac{\left(1-t_{a, e}\right)}{f_{z b z b}\left(\frac{r}{\left(1-t_{b, e}\right)}\right)}\right)} \\
& \Leftrightarrow \frac{\partial K_{a}}{\partial t_{b, e}}=\frac{-1}{\left(1-t_{b, e}\right)} \cdot \frac{r}{\left.\frac{\left(1-t_{a, e}\right)}{f_{z a z a}\left(\frac{r}{\left(1-t_{a, e}\right)}\right)}+\frac{\left(1-t_{b, e}\right)}{f_{z b z b}\left(\frac{r}{\left(1-t_{b, e}\right)}\right)}\right)} \text {. }
\end{aligned}
$$

Da nach Gleichung (2.52)

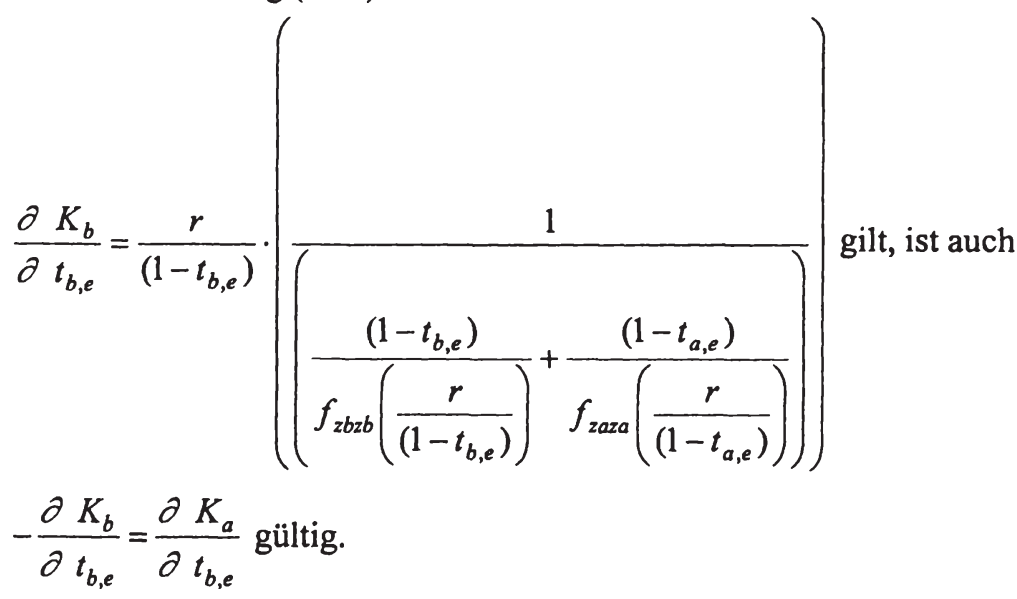

Claudia Hensberg - 978-3-631-75213-5

Downloaded from PubFactory at 01/11/2019 07:02:00AM

via free access 


\section{Anhang 4}

\section{Abschnitt a}

Gleichung (3.24) stellt eine implizite Funktion des Nettozinssatzes (r) dar:

$$
\bar{K}=f_{\mathrm{Pa}}\left(\frac{r}{E\left(G_{a}\right) \cdot\left(1-t_{a}\right)}\right)+f_{\mathrm{Pb}}\left(\frac{r}{E\left(G_{b}\right) \cdot\left(1-t_{b}\right)}\right) \text {. }
$$

Durch Ableitung der Funktion nach $t_{a}$ und Umformung ergibt sich:

$$
\begin{aligned}
& \frac{\partial \bar{K}}{\partial t_{a}}=f_{\mathrm{PaPa}}\left(\frac{r}{E\left(G_{a}\right) \cdot\left(1-t_{a}\right)}\right) \cdot \frac{\partial\left(\frac{r}{E\left(G_{a}\right) \cdot\left(1-t_{a}\right)}\right)}{\partial t_{a}} \\
& +f_{\mathrm{PbP} b}\left(\frac{r}{E\left(G_{b}\right) \cdot\left(1-t_{b}\right)}\right) \cdot \frac{\partial\left(\frac{r}{E\left(G_{b}\right) \cdot\left(1-t_{b}\right)}\right)}{\partial t_{a}} \\
& \Leftrightarrow 0=f_{\mathrm{PaPa}}\left(\frac{r}{E\left(G_{a}\right) \cdot\left(1-t_{a}\right)}\right) \cdot\left(\frac{\partial r}{\partial t_{a}} \cdot \frac{1}{E\left(G_{a}\right) \cdot\left(1-t_{a}\right)}+\frac{r}{E\left(G_{a}\right)} \cdot \frac{1}{\left(1-t_{a}\right)^{2}}\right) \\
& +f_{\mathrm{P} b \mathrm{~Pb}}\left(\frac{r}{E\left(G_{b}\right) \cdot\left(1-t_{b}\right)}\right) \cdot\left(\frac{\partial r}{\partial t_{a}} \cdot \frac{1}{E\left(G_{b}\right) \cdot\left(1-t_{b}\right)}\right) \\
& \Leftrightarrow 0=\frac{f_{\mathrm{PaPa}}\left(\frac{r}{E\left(G_{a}\right) \cdot\left(1-t_{a}\right)}\right)}{E\left(G_{a}\right) \cdot\left(1-t_{a}\right)} \cdot \frac{\partial r}{\partial t_{a}}+\frac{f_{\mathrm{PaPa}}\left(\frac{r}{E\left(G_{a}\right) \cdot\left(1-t_{a}\right)}\right)}{E\left(G_{a}\right) \cdot\left(1-t_{a}\right)} \cdot \frac{r}{\left(1-t_{a}\right)} \\
& +\frac{f_{\mathrm{P} b \mathrm{~Pb}}\left(\frac{r}{E\left(G_{b}\right) \cdot\left(1-t_{b}\right)}\right)}{E\left(G_{b}\right) \cdot\left(1-t_{b}\right)} \cdot \frac{\partial r}{\partial t_{a}} \\
& \Leftrightarrow\left(\frac{f_{\mathrm{PaPa}}\left(\frac{r}{E\left(G_{a}\right) \cdot\left(1-t_{a}\right)}\right)}{E\left(G_{a}\right) \cdot\left(1-t_{a}\right)}+\frac{f_{\mathrm{P} b \mathrm{~Pb}}\left(\frac{r}{E\left(G_{b}\right) \cdot\left(1-t_{b}\right)}\right)}{E\left(G_{b}\right) \cdot\left(1-t_{b}\right)}\right) \cdot \frac{\partial r}{\partial t_{a}} \\
& =\frac{-r}{\left(1-t_{a}\right)} \cdot \frac{f_{\mathrm{PaPa}}\left(\frac{r}{E\left(G_{a}\right) \cdot\left(1-t_{a}\right)}\right)}{E\left(G_{a}\right) \cdot\left(1-t_{a}\right)}
\end{aligned}
$$




$$
\begin{gathered}
\Leftrightarrow \frac{\partial r}{\partial t_{a}}=\frac{\frac{-r}{\left(1-t_{a}\right)} \cdot \frac{f_{\mathrm{PaPa}}\left(\frac{r}{E\left(G_{a}\right) \cdot\left(1-t_{a}\right)}\right)}{E\left(G_{a}\right) \cdot\left(1-t_{a}\right)}}{\left(\frac{r}{f_{\mathrm{PaP} a}\left(\frac{r}{E\left(G_{a}\right) \cdot\left(1-t_{a}\right)}\right)}+\frac{\left.f_{\mathrm{PbPb}}\left(\frac{r}{E\left(G_{b}\right) \cdot\left(1-t_{b}\right)}\right)\right)}{E\left(G_{b}\right) \cdot\left(1-t_{b}\right)}\right)} \\
\left.\Leftrightarrow \frac{\partial r}{\partial t_{a}}=\frac{-1}{\left(1-t_{a}\right)} \cdot \frac{\frac{\left.r \cdot t_{a}\right)}{\mathrm{PaPa}\left(\frac{r}{E\left(G_{a}\right) \cdot\left(1-t_{a}\right)}\right)}}{\left(\frac{E\left(G_{a}\right) \cdot\left(1-t_{a}\right)}{f_{\mathrm{PaPa} a}\left(\frac{r}{E\left(G_{a}\right) \cdot\left(1-t_{a}\right)}\right)}+\frac{f_{\mathrm{PbPb}}\left(\frac{r}{E\left(G_{b}\right) \cdot\left(1-t_{b}\right)}\right)}{E\left(G_{b}\right) \cdot\left(1-t_{b}\right)}\right)}\right)
\end{gathered}
$$$$
\Leftrightarrow \frac{\partial r}{\partial t_{a}}=\frac{-1}{\left(1-t_{a}\right)} \cdot \frac{r}{\left(1+\frac{\frac{f_{\mathrm{PbPb}}\left(\frac{r}{E\left(G_{b}\right) \cdot\left(1-t_{b}\right)}\right)}{E\left(G_{b}\right) \cdot\left(1-t_{b}\right)}}{\frac{f_{\mathrm{PaPa}}\left(\frac{r}{E\left(G_{a}\right) \cdot\left(1-t_{a}\right)}\right)}{E\left(G_{a}\right) \cdot\left(1-t_{a}\right)}}\right)}
$$

$$
\Leftrightarrow \frac{\partial r}{\partial t_{a}}=\frac{-1}{\left(1-t_{a}\right)} \cdot \frac{r}{\left.1+\frac{E\left(G_{a}\right) \cdot\left(1-t_{a}\right) \cdot f_{\mathrm{PbPb}}\left(\frac{r}{E\left(G_{b}\right) \cdot\left(1-t_{b}\right)}\right)}{E\left(G_{b}\right) \cdot\left(1-t_{b}\right) \cdot f_{\mathrm{PaPa}}\left(\frac{r}{E\left(G_{a}\right) \cdot\left(1-t_{a}\right)}\right)}\right)} .
$$

Berücksichtigt man, dass nach Gleichung (3.17) $f_{\mathrm{PiP} i}=\frac{1}{f_{K i K i}}$ gilt, so erhält man: 


$$
\frac{\partial r}{\partial t_{a}}=\frac{-1}{\left(1-t_{a}\right)} \cdot \frac{r}{\left(1+\frac{E\left(G_{a}\right) \cdot\left(1-t_{a}\right) \cdot f_{K a K a}\left(K_{a}\right)}{E\left(G_{b}\right) \cdot\left(1-t_{b}\right) \cdot f_{K b K b}\left(K_{b}\right)}\right)} .
$$

\section{Abschnitt b}

Nach Gleichung (3.15) und Gleichung (3.22) entspricht die Kapitalmenge $\left(\mathrm{K}_{\mathrm{a}}\right)$ :

$K_{a}=f_{\mathrm{Pa}}\left(\frac{r}{E\left(G_{a}\right) \cdot\left(1-t_{a}\right)}\right)$.

Durch Ableitung dieser Gleichung nach $\mathrm{t}_{\mathrm{a}}$ folgt:

$\frac{\partial K_{a}}{\partial t_{a}}=f_{\mathrm{PaPa}}\left(\frac{r}{E\left(G_{a}\right) \cdot\left(1-t_{a}\right)}\right) \cdot\left(\frac{\partial r}{\partial t_{a}} \cdot \frac{1}{E\left(G_{a}\right) \cdot\left(1-t_{a}\right)}+\frac{r}{E\left(G_{a}\right)} \cdot \frac{1}{\left(1-t_{a}\right)^{2}}\right)$.

Ersetzt man nun $\frac{\partial r}{\partial t_{a}}$ und formt um, so resultiert daraus:

$\begin{aligned} \frac{\partial K_{a}}{\partial t_{a}}= & \frac{f_{\mathrm{PaPa}}\left(\frac{r}{E\left(G_{a}\right) \cdot\left(1-t_{a}\right)}\right)}{E\left(G_{a}\right) \cdot\left(1-t_{a}\right)} \\ & \left(\begin{array}{l}\frac{-1}{\left(1-t_{a}\right)} \cdot \frac{r}{E\left(G_{a}\right) \cdot\left(1-t_{a}\right) \cdot f_{\mathrm{PbP} b}\left(\frac{r}{E\left(G_{b}\right) \cdot\left(1-t_{b}\right)}\right)} \\ 1+\frac{r}{E\left(G_{b}\right) \cdot\left(1-t_{b}\right) \cdot f_{\mathrm{PaP} a}\left(\frac{r}{E\left(G_{a}\right) \cdot\left(1-t_{a}\right)}\right)}\end{array}\right)\end{aligned}$ 


$$
\begin{aligned}
& \Leftrightarrow \frac{\partial K_{a}}{\partial t_{a}}=\frac{f_{\mathrm{PaPa}}\left(\frac{r}{E\left(G_{a}\right) \cdot\left(1-t_{a}\right)}\right)}{E\left(G_{a}\right) \cdot\left(1-t_{a}\right)} \cdot \frac{r}{\left(1-t_{a}\right)} \\
& \cdot\left(\frac{-1}{1+\frac{E\left(G_{a}\right) \cdot\left(1-t_{a}\right) \cdot f_{\mathrm{PbP} b}\left(\frac{r}{E\left(G_{b}\right) \cdot\left(1-t_{b}\right)}\right)}{E\left(G_{b}\right) \cdot\left(1-t_{b}\right) \cdot f_{\mathrm{PaPa}}\left(\frac{r}{E\left(G_{a}\right) \cdot\left(1-t_{a}\right)}\right)}}\right) \\
& \Leftrightarrow \frac{\partial K_{a}}{\partial t_{a}}=\frac{f_{\mathrm{PaPa}}\left(\frac{r}{E\left(G_{a}\right) \cdot\left(1-t_{a}\right)}\right)}{E\left(G_{a}\right) \cdot\left(1-t_{a}\right)} \cdot \frac{r}{\left(1-t_{a}\right)} \\
& \cdot\left(\frac{-1+\left(1+\frac{E\left(G_{a}\right) \cdot\left(1-t_{a}\right) \cdot f_{\mathrm{PbP}}\left(\frac{r}{E\left(G_{b}\right) \cdot\left(1-t_{b}\right)}\right)}{E\left(G_{b}\right) \cdot\left(1-t_{b}\right) \cdot f_{\mathrm{PaPa}}\left(\frac{r}{E\left(G_{a}\right) \cdot\left(1-t_{a}\right)}\right)}\right)}{1+\frac{E\left(G_{a}\right) \cdot\left(1-t_{a}\right) \cdot f_{\mathrm{PbP}}\left(\frac{r}{E\left(G_{b}\right) \cdot\left(1-t_{b}\right)}\right)}{E\left(G_{b}\right) \cdot\left(1-t_{b}\right) \cdot f_{\mathrm{PaPa}}\left(\frac{r}{E\left(G_{a}\right) \cdot\left(1-t_{a}\right)}\right)}}\right) \\
& \Leftrightarrow \frac{\partial K_{a}}{\partial t_{a}}=\frac{f_{\mathrm{PaPa}}\left(\frac{r}{E\left(G_{a}\right) \cdot\left(1-t_{a}\right)}\right)}{E\left(G_{a}\right) \cdot\left(1-t_{a}\right)} \cdot \frac{r}{\left(1-t_{a}\right)} .
\end{aligned}
$$




$$
\left(\frac{\frac{E\left(G_{a}\right) \cdot\left(1-t_{a}\right) \cdot f_{\mathrm{PbPb}}\left(\frac{r}{E\left(G_{b}\right) \cdot\left(1-t_{b}\right)}\right)}{E\left(G_{b}\right) \cdot\left(1-t_{b}\right) \cdot f_{\mathrm{PaPa}}\left(\frac{r}{E\left(G_{a}\right) \cdot\left(1-t_{a}\right)}\right)}}{1+\frac{E\left(G_{a}\right) \cdot\left(1-t_{a}\right) \cdot f_{\mathrm{PbP}}\left(\frac{r}{E\left(G_{b}\right) \cdot\left(1-t_{b}\right)}\right)}{E\left(G_{b}\right) \cdot\left(1-t_{b}\right) \cdot f_{\mathrm{PaPa}}\left(\frac{r}{E\left(G_{a}\right) \cdot\left(1-t_{a}\right)}\right)}}\right)
$$

$\Leftrightarrow \frac{\partial K_{a}}{\partial t_{a}}=\frac{r}{\left(1-t_{a}\right)} \cdot\left(\frac{\frac{f_{\mathrm{P} b \mathrm{P} b}\left(\frac{r}{E\left(G_{b}\right) \cdot\left(1-t_{b}\right)}\right)}{E\left(G_{b}\right) \cdot\left(1-t_{b}\right)}}{1+\frac{E\left(G_{a}\right) \cdot\left(1-t_{a}\right) \cdot f_{\mathrm{P} b \mathrm{~Pb}}\left(\frac{r}{E\left(G_{b}\right) \cdot\left(1-t_{b}\right)}\right)}{E\left(G_{b}\right) \cdot\left(1-t_{b}\right) \cdot f_{\mathrm{PaP} a}\left(\frac{r}{E\left(G_{a}\right) \cdot\left(1-t_{a}\right)}\right)}}\right)$

$\Leftrightarrow \frac{\partial K_{a}}{\partial t_{a}}=\frac{r}{\left(1-t_{a}\right)}$

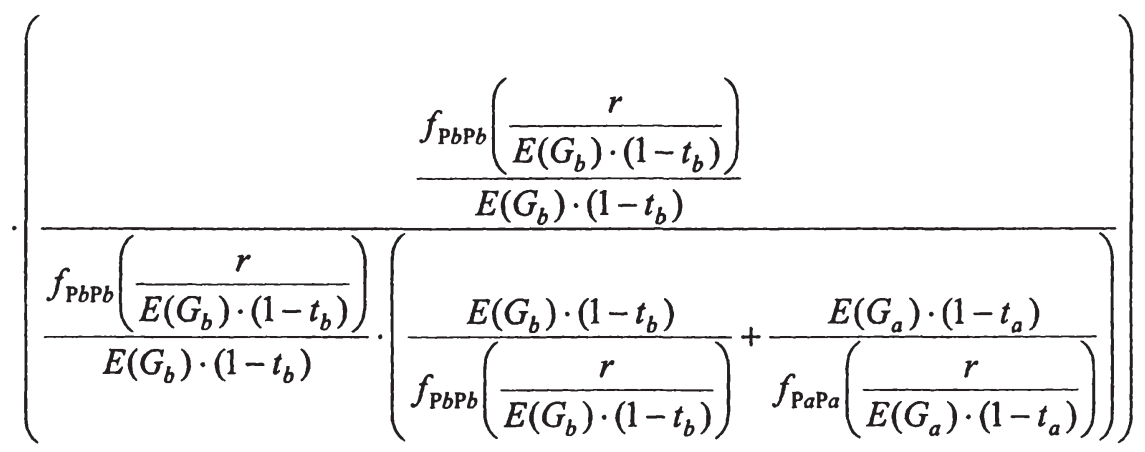




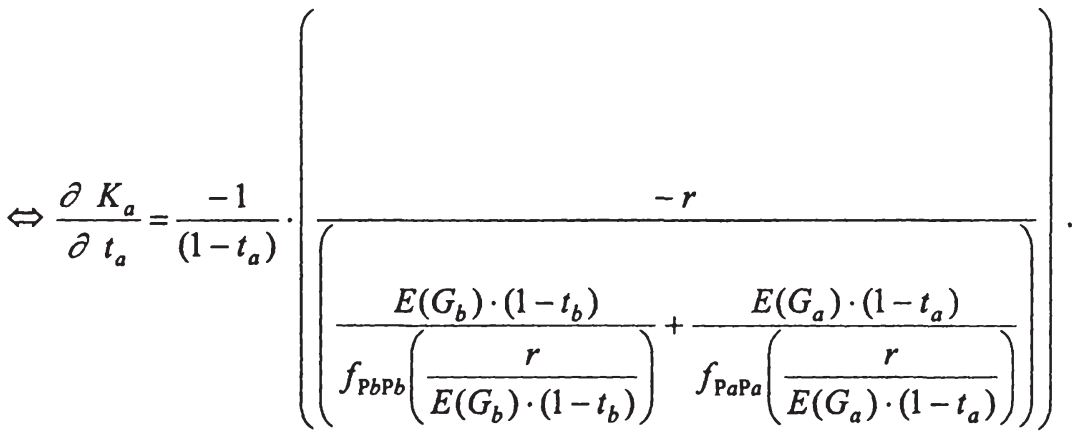

Berücksichtigt man Gleichung (3.17), dann gilt:

$$
\frac{\partial K_{a}}{\partial t_{a}}=\frac{-1}{\left(1-t_{a}\right)}\left(\frac{-r}{\left(E\left(G_{a}\right) \cdot\left(1-t_{a}\right) \cdot f_{K a K a}\left(K_{a}\right)+E\left(G_{b}\right) \cdot\left(1-t_{b}\right) \cdot f_{K b K b}\left(K_{b}\right)\right)}\right) \text {. }
$$

\section{Abschnitt c}

Nach Gleichung (3.15) und Gleichung (3.22) ist die Kapitalmenge $\left(\mathrm{K}_{\mathrm{b}}\right)$ festgelegt als:

$$
K_{b}=f_{\mathrm{Pb}}\left(\frac{r}{E\left(G_{b}\right) \cdot\left(1-t_{b}\right)}\right) \text {. }
$$

Durch Ableitung dieser Gleichung nach $t_{a}$ erhält man:

$$
\begin{aligned}
& \frac{\partial K_{b}}{\partial t_{a}}=f_{\mathrm{P} b \mathrm{~Pb}}\left(\frac{r}{E\left(G_{b}\right) \cdot\left(1-t_{b}\right)}\right) \cdot \frac{\partial r}{\partial t_{a}} \cdot \frac{1}{E\left(G_{b}\right) \cdot\left(1-t_{b}\right)} \\
& \text { bzw. } \frac{\partial K_{b}}{\partial t_{a}}=\frac{f_{\mathrm{PbP} b}\left(\frac{r}{E\left(G_{b}\right) \cdot\left(1-t_{b}\right)}\right)}{E\left(G_{b}\right) \cdot\left(1-t_{b}\right)} \cdot \frac{\partial r}{\partial t_{a}} .
\end{aligned}
$$

Ersetzt man nun $\frac{\partial r}{\partial t_{a}}$ und formt um, dann ergibt sich:

$\frac{\partial K_{b}}{\partial t_{a}}=\frac{f_{\mathrm{PbPb}}\left(\frac{r}{E\left(G_{b}\right) \cdot\left(1-t_{b}\right)}\right)}{E\left(G_{b}\right) \cdot\left(1-t_{b}\right)} \cdot \frac{-1}{\left(1-t_{a}\right)}$. 


$$
\begin{aligned}
& \frac{r}{\left(1+\frac{E\left(G_{a}\right) \cdot\left(1-t_{a}\right) \cdot f_{\mathrm{PbP}}\left(\frac{r}{E\left(G_{b}\right) \cdot\left(1-t_{b}\right)}\right)}{E\left(G_{b}\right) \cdot\left(1-t_{b}\right) \cdot f_{\mathrm{PaPa}}\left(\frac{r}{E\left(G_{a}\right) \cdot\left(1-t_{a}\right)}\right)}\right)} \\
& \Leftrightarrow \frac{\partial K_{b}}{\partial t_{a}}=\frac{-1}{\left(1-t_{a}\right)} \\
& \frac{f_{\mathrm{P} b \mathrm{~Pb}}\left(\frac{r}{E\left(G_{b}\right) \cdot\left(1-t_{b}\right)}\right)}{E\left(G_{b}\right) \cdot\left(1-t_{b}\right)} \cdot r \\
& \frac{f_{\mathrm{P} b \mathrm{~Pb}}\left(\frac{r}{E\left(G_{b}\right) \cdot\left(1-t_{b}\right)}\right)}{E\left(G_{b}\right) \cdot\left(1-t_{b}\right)} \cdot\left(\frac{E\left(G_{b}\right) \cdot\left(1-t_{b}\right)}{f_{\mathrm{P} b \mathrm{P} b}\left(\frac{r}{E\left(G_{b}\right) \cdot\left(1-t_{b}\right)}\right)}+\frac{E\left(G_{a}\right) \cdot\left(1-t_{a}\right)}{f_{\mathrm{PaP} a}\left(\frac{r}{E\left(G_{a}\right) \cdot\left(1-t_{a}\right)}\right)}\right) \\
& \Leftrightarrow \frac{\partial K_{b}}{\partial t_{a}}=\frac{-1}{\left(1-t_{a}\right)} \cdot \frac{r}{\left(\frac{E\left(G_{b}\right) \cdot\left(1-t_{b}\right)}{f_{\mathrm{PbP} b}\left(\frac{r}{E\left(G_{b}\right) \cdot\left(1-t_{b}\right)}\right)}+\frac{E\left(G_{a}\right) \cdot\left(1-t_{a}\right)}{f_{\mathrm{PaP} a}\left(\frac{r}{E\left(G_{a}\right) \cdot\left(1-t_{a}\right)}\right)}\right)} \text {. }
\end{aligned}
$$

Aus Gleichung (3.17) resultiert:

$$
\frac{\partial K_{b}}{\partial t_{a}}=\frac{-1}{\left(1-t_{a}\right)}\left(\frac{r}{\left(E\left(G_{a}\right) \cdot\left(1-t_{a}\right) \cdot f_{K a K a}\left(K_{a}\right)+E\left(G_{b}\right) \cdot\left(1-t_{b}\right) \cdot f_{K b K b}\left(K_{b}\right)\right)}\right) \text {. }
$$




\section{Anhang 5}

\section{Abschnitt a}

Gleichung (3.24) ist eine implizite Funktion des Nettozinssatzes (r):

$$
\bar{K}=f_{\mathrm{Pa}}\left(\frac{r}{E\left(G_{a}\right) \cdot\left(1-t_{a}\right)}\right)+f_{\mathrm{Pb}}\left(\frac{r}{E\left(G_{b}\right) \cdot\left(1-t_{b}\right)}\right) \text {. }
$$

Durch Ableitung der Funktion nach $\mathrm{G}_{\mathrm{a}}$ und Umformung folgt:

$$
\begin{aligned}
& \frac{\partial \bar{K}}{\partial G_{a}}=f_{\mathrm{PaPa}}\left(\frac{r}{E\left(G_{a}\right) \cdot\left(1-t_{a}\right)}\right) \cdot\left(\frac{\partial r}{\partial G_{a}} \cdot \frac{1}{E\left(G_{a}\right) \cdot\left(1-t_{a}\right)}+\frac{r}{\left(1-t_{a}\right)} \cdot(-1) \cdot\left(E\left(G_{a}\right)\right)^{-2}\right. \\
& \left.\cdot \frac{d E\left(G_{a}\right)}{d G_{a}}\right)+f_{\mathrm{PbPb}}\left(\frac{r}{E\left(G_{b}\right) \cdot\left(1-t_{b}\right)}\right) \cdot\left(\frac{\partial r}{\partial G_{a}} \cdot \frac{1}{E\left(G_{b}\right) \cdot\left(1-t_{b}\right)}\right) \\
& \Leftrightarrow 0=f_{\mathrm{PaP} a}\left(\frac{r}{E\left(G_{a}\right) \cdot\left(1-t_{a}\right)}\right) \cdot\left(\frac{\partial r}{\partial G_{a}} \cdot \frac{1}{E\left(G_{a}\right) \cdot\left(1-t_{a}\right)}+\frac{-r}{\left(1-t_{a}\right) \cdot E\left(G_{a}\right)} \cdot \frac{\frac{d E\left(G_{a}\right)}{d G_{a}}}{E\left(G_{a}\right)}\right) \\
& +f_{\mathrm{PbPb}}\left(\frac{r}{E\left(G_{b}\right) \cdot\left(1-t_{b}\right)}\right) \cdot\left(\frac{\partial r}{\partial G_{a}} \cdot \frac{1}{E\left(G_{b}\right) \cdot\left(1-t_{b}\right)}\right) \\
& \Leftrightarrow 0=\frac{f_{\mathrm{PaPa}}\left(\frac{r}{E\left(G_{a}\right) \cdot\left(1-t_{a}\right)}\right)}{E\left(G_{a}\right) \cdot\left(1-t_{a}\right)} \cdot\left(\frac{\partial r}{\partial G_{a}}-r \cdot \frac{\frac{d E\left(G_{a}\right)}{d G_{a}}}{E\left(G_{a}\right)}\right) \\
& +\frac{f_{\mathrm{P} b \mathrm{~Pb}}\left(\frac{r}{E\left(G_{b}\right) \cdot\left(1-t_{b}\right)}\right)}{E\left(G_{b}\right) \cdot\left(1-t_{b}\right)} \cdot \frac{\partial r}{\partial G_{a}} \\
& \Leftrightarrow 0=\frac{f_{\mathrm{PaPa}}\left(\frac{r}{E\left(G_{a}\right) \cdot\left(1-t_{a}\right)}\right)}{E\left(G_{a}\right) \cdot\left(1-t_{a}\right)} \cdot \frac{\partial r}{\partial G_{a}}-r \cdot \frac{\frac{d E\left(G_{a}\right)}{d G_{a}}}{E\left(G_{a}\right)} \cdot \frac{f_{\mathrm{PaPa}}\left(\frac{r}{E\left(G_{a}\right) \cdot\left(1-t_{a}\right)}\right)}{E\left(G_{a}\right) \cdot\left(1-t_{a}\right)} \\
& +\frac{f_{\mathrm{P} b \mathrm{~Pb}}\left(\frac{r}{E\left(G_{b}\right) \cdot\left(1-t_{b}\right)}\right)}{E\left(G_{b}\right) \cdot\left(1-t_{b}\right)} \cdot \frac{\partial r}{\partial G_{a}}
\end{aligned}
$$




$$
\begin{aligned}
& \Leftrightarrow 0=\left(\frac{f_{\mathrm{PaPa}}\left(\frac{r}{E\left(G_{a}\right) \cdot\left(1-t_{a}\right)}\right)}{E\left(G_{a}\right) \cdot\left(1-t_{a}\right)}+\frac{f_{\mathrm{PbPb}}\left(\frac{r}{E\left(G_{b}\right) \cdot\left(1-t_{b}\right)}\right)}{E\left(G_{b}\right) \cdot\left(1-t_{b}\right)}\right) \cdot \frac{\partial r}{\partial G_{a}} \\
& -r \cdot \frac{\frac{d E\left(G_{a}\right)}{d G_{a}}}{E\left(G_{a}\right)} \cdot \frac{f_{\mathrm{PaPa}}\left(\frac{r}{E\left(G_{a}\right) \cdot\left(1-t_{a}\right)}\right)}{E\left(G_{a}\right) \cdot\left(1-t_{a}\right)} \\
& \Leftrightarrow\left(\frac{f_{\mathrm{PaPa} a}\left(\frac{r}{E\left(G_{a}\right) \cdot\left(1-t_{a}\right)}\right)}{E\left(G_{a}\right) \cdot\left(1-t_{a}\right)}+\frac{f_{\mathrm{PbPb}}\left(\frac{r}{E\left(G_{b}\right) \cdot\left(1-t_{b}\right)}\right)}{E\left(G_{b}\right) \cdot\left(1-t_{b}\right)}\right) \cdot \frac{\partial r}{\partial G_{a}} \\
& =r \cdot \frac{\frac{d E\left(G_{a}\right)}{d G_{a}}}{E\left(G_{a}\right)} \cdot \frac{f_{\mathrm{PaP} a}\left(\frac{r}{E\left(G_{a}\right) \cdot\left(1-t_{a}\right)}\right)}{E\left(G_{a}\right) \cdot\left(1-t_{a}\right)} \\
& \Leftrightarrow \frac{\partial r}{\partial G_{a}}=\frac{r \cdot \frac{\frac{d E\left(G_{a}\right)}{d G_{a}}}{E\left(G_{a}\right)} \cdot \frac{f_{\mathrm{PaPa} a}\left(\frac{r}{E\left(G_{a}\right) \cdot\left(1-t_{a}\right)}\right)}{E\left(G_{a}\right) \cdot\left(1-t_{a}\right)}}{\left.\left(\frac{r}{\mathrm{PaPa}\left(\frac{r}{E\left(G_{a}\right) \cdot\left(1-t_{a}\right)}\right)}\right)+\frac{f_{\mathrm{PPPb}}\left(\frac{r}{E\left(G_{b}\right) \cdot\left(1-t_{b}\right)}\right)}{E\left(G_{a}\right) \cdot\left(1-t_{a}\right)}\right)} \\
& \Leftrightarrow \frac{\partial r}{\partial G_{a}}=\frac{r \cdot \frac{\frac{d E\left(G_{a}\right)}{d G_{a}}}{E\left(G_{a}\right)} \cdot \frac{f_{\mathrm{PaPa} a}\left(\frac{r}{E\left(G_{a}\right) \cdot\left(1-t_{a}\right)}\right)}{E\left(G_{a}\right) \cdot\left(1-t_{a}\right)}}{\frac{f_{\mathrm{PaPa}}\left(\frac{r}{E\left(G_{a}\right) \cdot\left(1-t_{a}\right)}\right)}{E\left(G_{a}\right) \cdot\left(1-t_{a}\right)} \cdot\left(1+\frac{\frac{f_{\mathrm{PbP}}\left(\frac{r}{E\left(G_{b}\right) \cdot\left(1-t_{b}\right)}\right)}{E\left(G_{b}\right) \cdot\left(1-t_{b}\right)}}{\left.\frac{f_{\mathrm{PaPa}}\left(\frac{r}{E\left(G_{a}\right) \cdot\left(1-t_{a}\right)}\right)}{E\left(G_{a}\right) \cdot\left(1-t_{a}\right)}\right)}\right.}
\end{aligned}
$$




$$
\begin{aligned}
& \Leftrightarrow \frac{\partial r}{\partial G_{a}}=\frac{\frac{\frac{d E\left(G_{a}\right)}{d G_{a}} \cdot r}{E\left(G_{a}\right)}}{\left(1+\frac{\frac{r}{\mathrm{PbPb}\left(\frac{E\left(G_{b}\right) \cdot\left(1-t_{b}\right)}{E\left(G_{b}\right) \cdot\left(1-t_{b}\right)}\right)}}{\frac{f_{\mathrm{PaPa}}\left(\frac{r}{E\left(G_{a}\right) \cdot\left(1-t_{a}\right)}\right)}{E\left(G_{a}\right) \cdot\left(1-t_{a}\right)}}\right)} \\
& \Leftrightarrow \frac{\partial r}{\partial G_{a}}=\frac{\frac{d E\left(G_{a}\right)}{d G_{a}}}{E\left(G_{a}\right)} \cdot \frac{r}{\left.1+\frac{E\left(G_{a}\right) \cdot\left(1-t_{a}\right) \cdot f_{\mathrm{PbP} b}\left(\frac{r}{E\left(G_{b}\right) \cdot\left(1-t_{b}\right)}\right)}{E\left(G_{b}\right) \cdot\left(1-t_{b}\right) \cdot f_{\mathrm{PaPa}}\left(\frac{r}{E\left(G_{a}\right) \cdot\left(1-t_{a}\right)}\right)}\right)} .
\end{aligned}
$$

Aus der Berücksichtigung von Gleichung (3.17) ergibt sich:

$$
\frac{\partial r}{\partial G_{a}}=\frac{\frac{d E\left(G_{a}\right)}{d G_{a}}}{E\left(G_{a}\right)} \cdot \frac{r}{\left(1+\frac{E\left(G_{a}\right) \cdot\left(1-t_{a}\right) \cdot f_{K a K a}\left(K_{a}\right)}{E\left(G_{b}\right) \cdot\left(1-t_{b}\right) \cdot f_{K b K b}\left(K_{b}\right)}\right)} \text {. }
$$

\section{Abschnitt b}

Nach Gleichung (3.15) in Verbindung mit Gleichung (3.22) entspricht die Kapitalmenge $\left(\mathrm{K}_{\mathrm{a}}\right)$ :

$$
K_{a}=f_{\mathrm{Pa}}\left(\frac{r}{E\left(G_{a}\right) \cdot\left(1-t_{a}\right)}\right) \text {. }
$$

Durch Ableitung dieser Gleichung nach $\mathrm{G}_{\mathrm{a}}$ und Umformung erhält man: 


$$
\begin{aligned}
& \frac{\partial K_{a}}{\partial G_{a}}=f_{\mathrm{PaPa}}\left(\frac{r}{E\left(G_{a}\right) \cdot\left(1-t_{a}\right)}\right) \\
& \cdot\left(\frac{\partial r}{\partial G_{a}} \cdot \frac{1}{E\left(G_{a}\right) \cdot\left(1-t_{a}\right)}+\frac{r}{\left(1-t_{a}\right)} \cdot(-1) \cdot E\left(G_{a}\right)^{-2} \cdot \frac{d E\left(G_{a}\right)}{d G_{a}}\right) \\
& \Leftrightarrow \frac{\partial K_{a}}{\partial G_{a}}=f_{\mathrm{PaPa}}\left(\frac{r}{E\left(G_{a}\right) \cdot\left(1-t_{a}\right)}\right) \\
& \\
& \cdot\left(\frac{\partial r}{\partial G_{a}} \cdot \frac{1}{E\left(G_{a}\right) \cdot\left(1-t_{a}\right)}+\frac{1}{E\left(G_{a}\right) \cdot\left(1-t_{a}\right)} \cdot \frac{-r \cdot \frac{d E\left(G_{a}\right)}{d G_{a}}}{E\left(G_{a}\right)}\right) \\
& \Leftrightarrow \frac{\partial K_{a}}{\partial G_{a}}=\frac{f_{\mathrm{PaPa}}\left(\frac{r}{E\left(G_{a}\right) \cdot\left(1-t_{a}\right)}\right)}{E\left(G_{a}\right) \cdot\left(1-t_{a}\right)} \cdot\left(\frac{\partial r}{\partial G_{a}}+\frac{-r \cdot \frac{d E\left(G_{a}\right)}{d G_{a}}}{E\left(G_{a}\right)}\right) .
\end{aligned}
$$

Ersetzt man nun $\frac{\partial r}{\partial G_{a}}$ und formt um, so resultiert daraus:

$$
\left.\begin{array}{rl}
\frac{\partial K_{a}}{\partial G_{a}}= & \frac{f_{\mathrm{PaPa}}\left(\frac{r}{E\left(G_{a}\right) \cdot\left(1-t_{a}\right)}\right) \frac{\frac{d E\left(G_{a}\right)}{d G_{a}}}{E\left(G_{a}\right)} \cdot r}{E\left(G_{a}\right) \cdot\left(1-t_{a}\right)} \\
& \cdot \frac{1}{\left(\frac{E\left(G_{a}\right) \cdot\left(1-t_{a}\right) \cdot f_{\mathrm{PbP} b}\left(\frac{r}{E\left(G_{b}\right) \cdot\left(1-t_{b}\right)}\right)}{E\left(G_{b}\right) \cdot\left(1-t_{b}\right) \cdot f_{\mathrm{PaPa} a}\left(\frac{r}{E\left(G_{a}\right) \cdot\left(1-t_{a}\right)}\right)}\right)}-1
\end{array}\right)
$$




$$
\begin{aligned}
& \Leftrightarrow \frac{\partial K_{a}}{\partial G_{a}}=\frac{f_{\mathrm{PaPa}}\left(\frac{r}{E\left(G_{a}\right) \cdot\left(1-t_{a}\right)}\right)}{E\left(G_{a}\right) \cdot\left(1-t_{a}\right)} \cdot \frac{\frac{d E\left(G_{a}\right)}{d G_{a}}}{E\left(G_{a}\right)} \cdot r \\
& \left(\begin{array}{c}
-\frac{E\left(G_{a}\right) \cdot\left(1-t_{a}\right) \cdot f_{\mathrm{PbP} b}\left(\frac{r}{E\left(G_{b}\right) \cdot\left(1-t_{b}\right)}\right)}{E\left(G_{b}\right) \cdot\left(1-t_{b}\right) \cdot f_{\mathrm{PaPa}}\left(\frac{r}{E\left(G_{a}\right) \cdot\left(1-t_{a}\right)}\right)} \\
\left(\begin{array}{c}
E\left(G_{a}\right) \cdot\left(1-t_{a}\right) \cdot f_{\mathrm{PbP} b}\left(\frac{r}{E\left(G_{b}\right) \cdot\left(1-t_{b}\right)}\right) \\
E\left(G_{b}\right) \cdot\left(1-t_{b}\right) \cdot f_{\mathrm{PaPa}}\left(\frac{r}{E\left(G_{a}\right) \cdot\left(1-t_{a}\right)}\right)
\end{array}\right)
\end{array}\right.
\end{aligned}
$$$$
\Leftrightarrow \frac{\partial K_{a}}{\partial G_{a}}=\frac{\frac{d E\left(G_{a}\right)}{d G_{a}}}{E\left(G_{a}\right)} \cdot r \cdot\left(\frac{f_{\mathrm{PbP} b}\left(\frac{r}{E\left(G_{b}\right) \cdot\left(1-t_{b}\right)}\right)}{E\left(G_{b}\right) \cdot\left(1-t_{b}\right)}\left(\frac{E\left(G_{a}\right) \cdot\left(1-t_{a}\right) \cdot f_{\mathrm{PbP} b}\left(\frac{r}{E\left(G_{b}\right) \cdot\left(1-t_{b}\right)}\right)}{\left.1+\frac{r}{E\left(G_{b}\right) \cdot\left(1-t_{b}\right) \cdot f_{\mathrm{PaPa}}\left(\frac{r}{E\left(G_{a}\right) \cdot\left(1-t_{a}\right)}\right)}\right)}\right)\right.
$$

$\Leftrightarrow \frac{\partial K_{a}}{\partial G_{a}}=\frac{\frac{d E\left(G_{a}\right)}{d G_{a}}}{E\left(G_{a}\right)} \cdot r$

$$
\left.\left(\frac{-\frac{f_{\mathrm{P} b \mathrm{~Pb}}\left(\frac{r}{E\left(G_{b}\right) \cdot\left(1-t_{b}\right)}\right)}{E\left(G_{b}\right) \cdot\left(1-t_{b}\right)}}{\frac{f_{\mathrm{P} b \mathrm{~Pb}}\left(\frac{r}{E\left(G_{b}\right) \cdot\left(1-t_{b}\right)}\right)}{E\left(G_{b}\right) \cdot\left(1-t_{b}\right)} \cdot\left(\frac{E\left(G_{b}\right) \cdot\left(1-t_{b}\right)}{f_{\mathrm{P} b \mathrm{~Pb}}\left(\frac{r}{E\left(G_{b}\right) \cdot\left(1-t_{b}\right)}\right)}+\frac{E\left(G_{a}\right) \cdot\left(1-t_{a}\right)}{f_{\mathrm{PaPa}}\left(\frac{r}{E\left(G_{a}\right) \cdot\left(1-t_{a}\right)}\right)}\right)}\right)\right)
$$




$$
\Leftrightarrow \frac{\partial K_{a}}{\partial G_{a}}=\frac{\frac{d E\left(G_{a}\right)}{d G_{a}}}{E\left(G_{a}\right)} \cdot\left(\frac{-r}{\left(\frac{E\left(G_{b}\right) \cdot\left(1-t_{b}\right)}{f_{\mathrm{PbPb}}\left(\frac{r}{E\left(G_{b}\right) \cdot\left(1-t_{b}\right)}\right)}+\frac{E\left(G_{a}\right) \cdot\left(1-t_{a}\right)}{f_{\mathrm{PPP} a}\left(\frac{r}{E\left(G_{a}\right) \cdot\left(1-t_{a}\right)}\right)}\right)}\right) .
$$

Unter Beachtung von Gleichung (3.17) kann auch geschrieben werden:

$$
\frac{\partial K_{a}}{\partial G_{a}}=\frac{\frac{d E\left(G_{a}\right)}{d G_{a}}}{E\left(G_{a}\right)} \cdot\left(\frac{-r}{E\left(G_{a}\right) \cdot\left(1-t_{a}\right) \cdot f_{K a K a}\left(K_{a}\right)+E\left(G_{b}\right) \cdot\left(1-t_{b}\right) \cdot f_{K b K b}\left(K_{b}\right)}\right) \text {. }
$$

\section{Abschnitt c}

Nach Gleichung (3.15) und Gleichung (3.22) ist die Kapitalmenge $\left(\mathrm{K}_{\mathrm{b}}\right)$ gegeben als:

$K_{b}=f_{\mathrm{Pb}}\left(\frac{r}{E\left(G_{b}\right) \cdot\left(1-t_{b}\right)}\right)$.

Durch Ableitung dieser Gleichung nach $\mathrm{G}_{\mathrm{a}}$ ergibt sich:

$\frac{\partial K_{b}}{\partial G_{a}}=f_{\mathrm{PbPb}}\left(\frac{r}{E\left(G_{b}\right) \cdot\left(1-t_{b}\right)}\right) \cdot \frac{\partial r}{\partial G_{a}} \cdot \frac{1}{E\left(G_{b}\right) \cdot\left(1-t_{b}\right)}$

bzw. $\frac{\partial K_{b}}{\partial G_{a}}=\frac{f_{\mathrm{PbPb}}\left(\frac{r}{E\left(G_{b}\right) \cdot\left(1-t_{b}\right)}\right)}{E\left(G_{b}\right) \cdot\left(1-t_{b}\right)} \cdot \frac{\partial r}{\partial G_{a}}$.

Ersetzt man nun $\frac{\partial r}{\partial G_{a}}$ und formt um, so folgt: 


$$
\begin{aligned}
& \frac{\partial K_{b}}{\partial G_{a}}=\frac{f_{\mathrm{P} b \mathrm{~Pb}}\left(\frac{r}{E\left(G_{b}\right) \cdot\left(1-t_{b}\right)}\right)}{E\left(G_{b}\right) \cdot\left(1-t_{b}\right)} \cdot \frac{\frac{d E\left(G_{a}\right)}{d G_{a}}}{E\left(G_{a}\right)} \\
& \cdot \frac{r}{1+\frac{E\left(G_{a}\right) \cdot\left(1-t_{a}\right) \cdot f_{\mathrm{PbPb}}\left(\frac{r}{E\left(G_{b}\right) \cdot\left(1-t_{b}\right)}\right)}{E\left(G_{b}\right) \cdot\left(1-t_{b}\right) \cdot f_{\mathrm{PaPa}}\left(\frac{r}{E\left(G_{a}\right) \cdot\left(1-t_{a}\right)}\right)}} \\
& \Leftrightarrow \frac{\partial K_{b}}{\partial G_{a}}=\frac{\frac{d E\left(G_{a}\right)}{d G_{a}}}{E\left(G_{a}\right)} \\
& r \cdot \frac{f_{\mathrm{P} b \mathrm{~Pb}}\left(\frac{r}{E\left(G_{b}\right) \cdot\left(1-t_{b}\right)}\right)}{E\left(G_{b}\right) \cdot\left(1-t_{b}\right)} \\
& \frac{f_{\mathrm{PbPb}}\left(\frac{r}{E\left(G_{b}\right) \cdot\left(1-t_{b}\right)}\right)}{E\left(G_{b}\right) \cdot\left(1-t_{b}\right)} \cdot\left(\frac{E\left(G_{b}\right) \cdot\left(1-t_{b}\right)}{f_{\mathrm{PbP} b}\left(\frac{r}{E\left(G_{b}\right) \cdot\left(1-t_{b}\right)}\right)}+\frac{E\left(G_{a}\right) \cdot\left(1-t_{a}\right)}{f_{\mathrm{PaPa}}\left(\frac{r}{E\left(G_{a}\right) \cdot\left(1-t_{a}\right)}\right)}\right) \\
& \Leftrightarrow \frac{\partial K_{b}}{\partial G_{a}}=\frac{\frac{d E\left(G_{a}\right)}{d G_{a}}}{E\left(G_{a}\right)} \cdot \frac{r}{\left(\frac{E\left(G_{b}\right) \cdot\left(1-t_{b}\right)}{f_{\mathrm{PbPb}}\left(\frac{r}{E\left(G_{b}\right) \cdot\left(1-t_{b}\right)}\right)}+\frac{E\left(G_{a}\right) \cdot\left(1-t_{a}\right)}{f_{\mathrm{PaPa}}\left(\frac{r}{E\left(G_{a}\right) \cdot\left(1-t_{a}\right)}\right)}\right)} .
\end{aligned}
$$

Unter Berücksichtigung von Gleichung (3.17) erhält man:

$$
\frac{\partial K_{b}}{\partial G_{a}}=\frac{\frac{d E\left(G_{a}\right)}{d G_{a}}}{E\left(G_{a}\right)} \cdot \frac{r}{\left(E\left(G_{a}\right) \cdot\left(1-t_{a}\right) \cdot f_{K a K a}\left(K_{a}\right)+E\left(G_{b}\right) \cdot\left(1-t_{b}\right) \cdot f_{K b K b}\left(K_{b}\right)\right)} .
$$




\section{Anhang 6}

Eine Änderung der fiskalpolitischen Instrumente führt zu keinerlei Änderungen auf dem Kapitalmarkt, wenn gleichzeitig

(i) $\frac{\partial K_{a}}{\partial t_{a}} \cdot d t_{a}+\frac{\partial K_{a}}{\partial G_{a}} \cdot d G_{a}=0$

(ii) $\frac{\partial K_{b}}{\partial t_{a}} \cdot d t_{a}+\frac{\partial K_{b}}{\partial G_{a}} \cdot d G_{a}=0$

und

(iii) $\frac{\partial r}{\partial t_{a}} \cdot d t_{a}+\frac{\partial r}{\partial G_{a}} \cdot d G_{a}=0$

gilt. Unter Berücksichtigung von Gleichung (3.27) und Gleichung (3.34) entspricht Bedingung (i):

$$
\begin{aligned}
& 0=\frac{-1}{\left(1-t_{a}\right)} \cdot \frac{-r}{\left(E\left(G_{a}\right) \cdot\left(1-t_{a}\right) \cdot f_{K a K a}\left(K_{a}\right)+E\left(G_{b}\right) \cdot\left(1-t_{b}\right) \cdot f_{K b K b}\left(K_{b}\right)\right)} \cdot d t_{a} \\
& +\frac{\frac{d E\left(G_{a}\right)}{d G_{a}}}{E\left(G_{a}\right)} \cdot\left(\frac{-r}{E\left(G_{a}\right) \cdot\left(1-t_{a}\right) \cdot f_{K a K a}\left(K_{a}\right)+E\left(G_{b}\right) \cdot\left(1-t_{b}\right) \cdot f_{K b K b}\left(K_{b}\right)}\right) \cdot d G_{a} \\
& \Leftrightarrow 0=\left(\frac{-1}{\left(1-t_{a}\right)} \cdot d t_{a}+\frac{\frac{d E\left(G_{a}\right)}{d G_{a}}}{E\left(G_{a}\right)} \cdot d G_{a}\right) \\
& \left(\frac{-r}{E\left(G_{a}\right) \cdot\left(1-t_{a}\right) \cdot f_{K a K a}\left(K_{a}\right)+E\left(G_{b}\right) \cdot\left(1-t_{b}\right) \cdot f_{K b K b}\left(K_{b}\right)}\right) \\
& \Leftrightarrow 0=\left(\frac{-1}{\left(1-t_{a}\right)} \cdot d t_{a}+\frac{\frac{d E\left(G_{a}\right)}{d G_{a}}}{E\left(G_{a}\right)} \cdot d G_{a}\right)
\end{aligned}
$$

Unter Beachtung von Gleichung (3.30) und Gleichung (3.37) erhält man für Bedingung (ii) auch: 


$$
\begin{aligned}
& 0=\frac{-1}{\left(1-t_{a}\right)} \cdot \frac{r}{\left(E\left(G_{a}\right) \cdot\left(1-t_{a}\right) \cdot f_{K a K a}\left(K_{a}\right)+E\left(G_{b}\right) \cdot\left(1-t_{b}\right) \cdot f_{K b K b}\left(K_{b}\right)\right)} \cdot d t_{a} \\
& +\frac{\frac{d E\left(G_{a}\right)}{d G_{a}}}{E\left(G_{a}\right)} \cdot \frac{r}{\left(E\left(G_{a}\right) \cdot\left(1-t_{a}\right) \cdot f_{K a K a}\left(K_{a}\right)+E\left(G_{b}\right) \cdot\left(1-t_{b}\right) \cdot f_{K b K b}\left(K_{b}\right)\right)} \cdot d G_{a} \\
& \Leftrightarrow 0=\left(\frac{-1}{\left(1-t_{a}\right)} \cdot d t_{a}+\frac{\frac{d E\left(G_{a}\right)}{d G_{a}}}{E\left(G_{a}\right)} \cdot d G_{a}\right) \\
& \cdot\left(\frac{r}{E\left(G_{a}\right) \cdot\left(1-t_{a}\right) \cdot f_{K a K a}\left(K_{a}\right)+E\left(G_{b}\right) \cdot\left(1-t_{b}\right) \cdot f_{K b K b}\left(K_{b}\right)}\right) \\
& \Leftrightarrow 0=\left(\frac{-1}{\left(1-t_{a}\right)} \cdot d t_{a}+\frac{\frac{d E\left(G_{a}\right)}{d G_{a}}}{E\left(G_{a}\right)} \cdot d G_{a}\right)
\end{aligned}
$$

Berücksichtigt man Gleichung (3.25) und Gleichung (3.32), so resultiert aus Bedingung (iii):

$$
\begin{aligned}
& 0=\frac{-1}{\left(1-t_{a}\right)} \cdot \frac{r}{\left(1+\frac{E\left(G_{a}\right) \cdot\left(1-t_{a}\right) \cdot f_{K a K a}\left(K_{a}\right)}{E\left(G_{b}\right) \cdot\left(1-t_{b}\right) \cdot f_{K b K b}\left(K_{b}\right)}\right)} \cdot d t_{a} \\
& +\frac{\frac{d E\left(G_{a}\right)}{d G_{a}}}{E\left(G_{a}\right)} \cdot \frac{r}{\left(1+\frac{E\left(G_{a}\right) \cdot\left(1-t_{a}\right) \cdot f_{K a K a}\left(K_{a}\right)}{E\left(G_{b}\right) \cdot\left(1-t_{b}\right) \cdot f_{K b K b}\left(K_{b}\right)}\right)} \cdot d G_{a} \\
& \Leftrightarrow 0=\left(\frac{-1}{\left(1-t_{a}\right)} \cdot d t_{a}+\frac{\frac{d E\left(G_{a}\right)}{d G_{a}}}{E\left(G_{a}\right)} \cdot d G_{a}\right) \cdot\left(\frac{r}{\left(1+\frac{E\left(G_{a}\right) \cdot\left(1-t_{a}\right) \cdot f_{K a K a}\left(K_{a}\right)}{E\left(G_{b}\right) \cdot\left(1-t_{b}\right) \cdot f_{K b K b}\left(K_{b}\right)}\right)}\right) \\
& \Leftrightarrow 0=\left(\frac{-1}{\left(1-t_{a}\right)} \cdot d t_{a}+\frac{\frac{d E\left(G_{a}\right)}{d G_{a}}}{E\left(G_{a}\right)} \cdot d G_{a}\right)
\end{aligned}
$$




\section{Anhang 7}

\section{Abschnitt a}

Gleichung (3.62) ist eine implizite Funktion des Nettozinssatzes (r):

$\bar{K}=f_{\mathrm{Pa}}\left(\frac{r}{e^{I_{a}}}\right)+f_{\mathrm{Pb}}\left(\frac{r}{e^{I_{b}}}\right)$.

Aus der Ableitung der Funktion nach $\mathrm{I}_{\mathrm{a}}$ und Umformung folgt:

$\frac{\partial \bar{K}}{\partial I_{a}}=f_{\mathrm{PaPa} a}\left(\frac{r}{e^{I_{a}}}\right) \cdot \frac{\partial\left(\frac{r}{e^{I_{a}}}\right)}{\partial I_{a}}+f_{\mathrm{PbPb}}\left(\frac{r}{e^{I_{b}}}\right) \cdot \frac{\partial\left(\frac{r}{e^{I_{b}}}\right)}{\partial I_{a}}$

$\Leftrightarrow 0=f_{\mathrm{PaP} a}\left(\frac{r}{e^{I_{a}}}\right) \cdot\left(\frac{\partial r}{\partial I_{a}} \cdot \frac{1}{e^{I_{a}}}+r \cdot(-1) \cdot\left(e^{I_{a}}\right)^{-2} \cdot e^{I_{a}}\right)+f_{\mathrm{PbP} b}\left(\frac{r}{e^{I_{b}}}\right) \cdot\left(\frac{\partial r}{\partial I_{a}} \cdot \frac{1}{e^{I b}}\right)$

$\Leftrightarrow 0=f_{\mathrm{PaP} a}\left(\frac{r}{e^{I_{a}}}\right) \cdot\left(\frac{\partial r}{\partial I_{a}} \cdot \frac{1}{e^{I_{a}}}-r \cdot \frac{1}{e^{I_{a}}}\right)+f_{\mathrm{PbP} b}\left(\frac{r}{e^{I_{b}}}\right) \cdot\left(\frac{\partial r}{\partial I_{a}} \cdot \frac{1}{e^{I b}}\right)$

$\Leftrightarrow 0=\frac{f_{\mathrm{PaPa}}\left(\frac{r}{e^{I_{a}}}\right)}{e^{I_{a}}} \cdot\left(\frac{\partial r}{\partial I_{a}}\right)-\frac{f_{\mathrm{PaPa}}\left(\frac{r}{e^{I_{a}}}\right)}{e^{I_{a}}} \cdot r+\frac{f_{\mathrm{P} b \mathrm{~Pb}}\left(\frac{r}{e^{I_{b}}}\right)}{e^{I_{b}}} \cdot\left(\frac{\partial r}{\partial I_{a}}\right)$

$\Leftrightarrow \frac{f_{\mathrm{PaPa}}\left(\frac{r}{e^{I_{a}}}\right)}{e^{I_{a}}} \cdot r=\left(\frac{f_{\mathrm{PaPa}}\left(\frac{r}{e^{I_{a}}}\right)}{e^{I_{a}}}+\frac{f_{\mathrm{PbPb}}\left(\frac{r}{e^{I_{b}}}\right)}{e^{I_{b}}}\right) \cdot\left(\frac{\partial r}{\partial I_{a}}\right)$

$\Leftrightarrow \frac{\partial r}{\partial I_{a}}=\frac{\frac{f_{\mathrm{PaP} a}\left(\frac{r}{e^{I_{a}}}\right)}{e^{I_{a}}} \cdot r}{\left(\frac{f_{\mathrm{PaPa}}\left(\frac{r}{e^{I_{a}}}\right)}{e^{I_{a}}}+\frac{f_{\mathrm{Pb} b}\left(\frac{r}{e^{I_{b}}}\right)}{e^{I_{b}}}\right)}$ 


$$
\left.\Leftrightarrow \frac{\partial r}{\partial I_{a}}=\frac{\frac{f_{\mathrm{PaPa}}\left(\frac{r}{e^{I_{a}}}\right)}{e_{\mathrm{PaPa}}\left(\frac{r}{e^{I_{a}}}\right)} \cdot\left(1+\frac{\frac{f_{\mathrm{PbPb}}\left(\frac{r}{e^{I_{b}}}\right)}{e^{I_{a}}}}{\frac{e^{I_{b}}}{f_{\mathrm{PaPa}}\left(\frac{r}{e^{I_{a}}}\right)}}\right)}{e^{I_{a}}}\right)
$$

$\Leftrightarrow \frac{\partial r}{\partial I_{a}}=\frac{r}{\left(1+\frac{\frac{f_{\mathrm{PbPb}}\left(\frac{r}{e^{I_{b}}}\right)}{e^{I_{b}}}}{\frac{f_{\mathrm{PaPa}}\left(\frac{r}{e^{I_{a}}}\right)}{e^{I_{a}}}}\right)}$

$$
\Leftrightarrow \frac{\partial r}{\partial I_{a}}=\frac{r}{\left(1+\frac{e^{I_{a}} \cdot f_{\mathrm{PbPb}}\left(\frac{r}{e^{I_{b}}}\right)}{e^{I_{b}} \cdot f_{\mathrm{PaPa}}\left(\frac{r}{e^{I_{a}}}\right)}\right)}
$$

Berücksichtigt man, dass nach Gleichung (3.17) $f_{\mathrm{P} i \mathrm{P} i}=\frac{1}{f_{K i K i}}$ gilt, so ergibt sich:

$$
\frac{\partial r}{\partial I_{a}}=\frac{r}{\left(1+\frac{e^{I_{a}} \cdot f_{K a K a}\left(K_{a}\right)}{e^{I_{b}} \cdot f_{K b K b}\left(K_{b}\right)}\right)}
$$

\section{Abschnitt b}

Nach Gleichung (3.15) und Gleichung (3.22) entspricht die Kapitalmenge $\left(\mathrm{K}_{\mathrm{a}}\right)$ :

$$
K_{a}=f_{\mathrm{Pa}}\left(\frac{r}{E\left(G_{a}\right) \cdot\left(1-t_{a}\right)}\right) \text {. }
$$


Unter Beachtung von Gleichung (3.49) kann diese in Abhängigkeit des fiskalpolitischen Instrumenten-Indexes ausgedrückt werden:

$$
K_{a}=f_{\mathrm{Pa}}\left(\frac{r}{e^{I_{a}}}\right) \text {. }
$$

Durch Ableitung dieser Gleichung nach $\mathrm{I}_{\mathrm{a}}$ erhält man:

$$
\begin{aligned}
& \frac{\partial K_{a}}{\partial I_{a}}=f_{\mathrm{PaPa}}\left(\frac{r}{e^{I_{a}}}\right) \cdot \frac{\partial\left(\frac{r}{e^{I_{a}}}\right)}{\partial I_{a}} \\
& \Leftrightarrow \frac{\partial K_{a}}{\partial I_{a}}=f_{\mathrm{PaPa}}\left(\frac{r}{e^{I_{a}}}\right) \cdot\left(\frac{\partial r}{\partial I_{a}} \cdot \frac{1}{e^{I_{a}}}+r \cdot(-1) \cdot\left(e^{I_{a}}\right)^{-2} \cdot e^{I_{a}}\right) \\
& \Leftrightarrow \frac{\partial K_{a}}{\partial I_{a}}=f_{\mathrm{PaPa}}\left(\frac{r}{e^{I_{a}}}\right) \cdot\left(\frac{\partial r}{\partial I_{a}} \cdot \frac{1}{e^{I_{a}}}-r \cdot \frac{1}{e^{I_{a}}}\right) \\
& \Leftrightarrow \frac{\partial K_{a}}{\partial I_{a}}=\frac{f_{\mathrm{PaPa}}\left(\frac{r}{e^{I_{a}}}\right)}{e^{I_{a}}} \cdot\left(\left(\frac{\partial r}{\partial I_{a}}\right)-r\right) .
\end{aligned}
$$

Ersetzt man nun $\frac{\partial r}{\partial I_{a}} \operatorname{durch} \frac{r}{\left(1+\frac{e^{I_{a}} \cdot f_{\mathrm{PbPb}}\left(\frac{r}{e^{I_{b}}}\right)}{e^{I_{b}} \cdot f_{\mathrm{PaPa}}\left(\frac{r}{e^{I_{a}}}\right)}\right)}$, so resultiert daraus:

$$
\frac{\partial K_{a}}{\partial I_{a}}=\frac{f_{\mathrm{PaPa}}\left(\frac{r}{e^{I_{a}}}\right)}{e^{I_{a}}} \cdot\left(\frac{r}{\left(1+\frac{e^{I_{a}} \cdot f_{\mathrm{P} b \mathrm{~Pb}}\left(\frac{r}{e^{I_{b}}}\right)}{e^{I_{b}} \cdot f_{\mathrm{PaPa}}\left(\frac{r}{e^{I_{a}}}\right)}\right)}-r\right)
$$




$$
\begin{aligned}
& \Leftrightarrow \frac{\partial K_{a}}{\partial I_{a}}=-r \cdot \frac{f_{\mathrm{PaPa}}\left(\frac{r}{e^{I_{a}}}\right)}{e^{I_{a}}} \cdot \frac{\left(\frac{e^{I_{a}} \cdot f_{\mathrm{PbPb}}\left(\frac{r}{e^{I_{b}}}\right)}{e^{I_{b}} \cdot f_{\mathrm{PaPa}}\left(\frac{r}{e^{I_{a}}}\right)}\right)}{\left(1+\frac{e^{I_{a}} \cdot f_{\mathrm{P} b \mathrm{~Pb}}\left(\frac{r}{e^{I_{b}}}\right)}{e^{I_{b}} \cdot f_{\mathrm{PaPa}}\left(\frac{r}{e^{I_{a}}}\right)}\right)} \\
& \Leftrightarrow \frac{\partial K_{a}}{\partial I_{a}}=-r \cdot \frac{\left(\frac{f_{\mathrm{PbP} b}\left(\frac{r}{e^{I_{b}}}\right)}{e^{I_{b}}}\right)}{\left(\frac{f_{\mathrm{P} b \mathrm{~Pb}}\left(\frac{r}{e^{I_{b}}}\right)}{e^{I_{b}}}\right) \cdot\left(\frac{e^{I_{b}}}{f_{\mathrm{P} b \mathrm{~Pb}}\left(\frac{r}{e^{I_{b}}}\right)}+\frac{e^{I_{a}}}{f_{\mathrm{PaPa}}\left(\frac{r}{e^{I_{a}}}\right)}\right)} \\
& \Leftrightarrow \frac{\partial K_{a}}{\partial I_{a}}=\frac{-r}{\left(\frac{e^{I_{b}}}{f_{\mathrm{P} b \mathrm{~Pb}}\left(\frac{r}{e^{I_{b}}}\right)}+\frac{e^{I_{a}}}{f_{\mathrm{PaPa}}\left(\frac{r}{e^{I_{a}}}\right)}\right)} .
\end{aligned}
$$

Wird nun beachtet, dass nach Gleichung (3.17) $f_{\mathrm{PiPi}}=\frac{1}{f_{K i K i}}$ vorliegt, so ergibt sich:$$
\frac{\partial K_{a}}{\partial I_{a}}=\frac{-r}{\left(e^{I_{a}} \cdot f_{K a K a}\left(K_{a}\right)+e^{I_{b}} \cdot f_{K b K b}\left(K_{b}\right)\right)} \text {. }
$$

\section{Abschnitt c}

Nach Gleichung (3.15) und Gleichung (3.22) ist die Kapitalmenge $\left(\mathrm{K}_{\mathrm{b}}\right)$ gegeben als: 
$K_{b}=f_{\mathrm{Pb}}\left(\frac{r}{E\left(G_{b}\right) \cdot\left(1-t_{b}\right)}\right)$.

Berücksichtigt man Gleichung (3.49), so lässt sich diese auch in Abhängigkeit des fiskalpolitischen Instrumenten-Indexes schreiben:

$K_{b}=f_{\mathrm{Pb}}\left(\frac{r}{e^{I_{b}}}\right)$.

Durch Ableitung dieser Gleichung nach $\mathrm{I}_{\mathrm{a}}$ erhält man:

$\frac{\partial K_{b}}{\partial I_{a}}=f_{\mathrm{P} b \mathrm{~Pb}}\left(\frac{r}{e^{I_{b}}}\right) \cdot \frac{\partial\left(\frac{r}{e^{I_{b}}}\right)}{\partial I_{a}}$
$\Leftrightarrow \frac{\partial K_{b}}{\partial I_{a}}=f_{\mathrm{PbPb}}\left(\frac{r}{e^{I_{b}}}\right) \cdot\left(\frac{\partial r}{\partial I_{a}} \cdot \frac{1}{e^{I_{b}}}\right)$

$\Leftrightarrow \frac{\partial K_{b}}{\partial I_{a}}=\frac{f_{\mathrm{PbPb}}\left(\frac{r}{e^{I_{b}}}\right)}{e^{I_{b}}} \cdot\left(\frac{\partial r}{\partial I_{a}}\right)$.

Ersetzt man nun $\frac{\partial r}{\partial I_{a}}$ entsprechend durch $\frac{r}{\left(1+\frac{e^{I_{a}} \cdot f_{\mathrm{PbP} b}\left(\frac{r}{e^{I_{b}}}\right)}{e^{I_{b}} \cdot f_{\mathrm{PaP} a}\left(\frac{r}{e^{I_{a}}}\right)}\right)}$, dann folgt:

$\frac{\partial K_{b}}{\partial I_{a}}=\frac{f_{\mathrm{PbPb}}\left(\frac{r}{e^{I_{b}}}\right)}{e^{I_{b}}} \cdot\left(\frac{r}{\left(1+\frac{e^{I_{a}} \cdot f_{\mathrm{P} b \mathrm{~Pb}}\left(\frac{r}{e^{I_{b}}}\right)}{e^{I_{b}} \cdot f_{\mathrm{PaPa}}\left(\frac{r}{e^{I_{a}}}\right)}\right)}\right)$ 
$\Leftrightarrow \frac{\partial K_{b}}{\partial I_{a}}=\frac{f_{\mathrm{PbPb}}\left(\frac{r}{e^{I_{b}}}\right)}{e^{I_{b}}} \cdot\left(\frac{r}{\frac{f_{\mathrm{PbP} b}\left(\frac{r}{e^{I_{b}}}\right)}{e^{I_{b}}} \cdot\left(\frac{e^{I_{b}}}{f_{\mathrm{PbPb}}\left(\frac{r}{e^{I_{b}}}\right)}+\frac{e^{I_{a}}}{f_{\mathrm{PaPa}}\left(\frac{r}{e^{I_{a}}}\right)}\right)}\right)$

$\Leftrightarrow \frac{\partial K_{b}}{\partial I_{a}}=\frac{r}{\left(\frac{e^{I_{b}}}{f_{\mathrm{PbPb}}\left(\frac{r}{e^{I_{b}}}\right)}+\frac{e^{I_{a}}}{f_{\mathrm{PaPa}}\left(\frac{r}{e^{I_{a}}}\right)}\right)}$.

Wird nun bedacht, dass nach Gleichung (3.17) $f_{\mathrm{PiPi}}=\frac{1}{f_{K I K I}}$ gilt, so ergibt sich:

$\frac{\partial K_{b}}{\partial I_{a}}=\frac{r}{\left(e^{I_{a}} \cdot f_{K a K a}\left(K_{a}\right)+e^{I_{b}} \cdot f_{K b K b}\left(K_{b}\right)\right)}$. 


\section{Anhang 8}

Die Kapitalmarktgleichgewichtsbedingung für Region a entspricht nach Gleichung (4.20):

$E\left(G_{a}\right) \cdot f_{K a}\left(K_{a}\right)=E\left(G_{b}\right) \cdot f_{K b}\left(\bar{K}-K_{a}\right)$.

Leitet man diese implizite Funktion nach $\mathrm{G}_{\mathrm{a}}$ ab, so erhält man:

$\frac{d E}{d G_{a}} \cdot f_{K a}\left(K_{a}\right)+E\left(G_{a}\right) \cdot f_{K a K a}\left(K_{a}\right) \cdot \frac{\partial K_{a}}{\partial G_{a}}=E\left(G_{b}\right) \cdot f_{K b K b}\left(\bar{K}-K_{a}\right) \cdot(-1) \cdot \frac{\partial K_{a}}{\partial G_{a}}$

$\Leftrightarrow \frac{d E}{d G_{a}} \cdot f_{K a}\left(K_{a}\right)=-\left(E\left(G_{b}\right) \cdot f_{K b K b}\left(\bar{K}-K_{a}\right) \cdot \frac{\partial K_{a}}{\partial G_{a}}+E\left(G_{a}\right) \cdot f_{K a K a}\left(K_{a}\right) \cdot \frac{\partial K_{a}}{\partial G_{a}}\right)$

$\Leftrightarrow \frac{d E}{d G_{a}} \cdot f_{K a}\left(K_{a}\right)=-\left(E\left(G_{b}\right) \cdot f_{K b K b}\left(\bar{K}-K_{a}\right)+E\left(G_{a}\right) \cdot f_{K a K a}\left(K_{a}\right)\right) \cdot \frac{\partial K_{a}}{\partial G_{a}}$.

Die Ableitung der Kapitalmenge $\left(\mathrm{K}_{\mathrm{a}}\right)$ nach der Menge an öffentlichem Zwischenprodukt $\left(G_{a}\right)$ ist demnach:

$$
\frac{\partial K_{a}}{\partial G_{a}}=\frac{\frac{d E}{d G_{a}} \cdot f_{K a}\left(K_{a}\right)}{-\left(E\left(G_{b}\right) \cdot f_{K b K b}\left(\bar{K}-K_{a}\right)+E\left(G_{a}\right) \cdot f_{K a K a}\left(K_{a}\right)\right)} .
$$




\section{Anhang 9}

Es soll gezeigt werden, dass die Reaktionsfunktionen der Leviathanregierungen $\tilde{t}_{K} \cdot \frac{d E}{d G_{a}} \cdot \frac{f_{K a}\left(K_{a}\right)}{-\left(E\left(G_{a}\right) \cdot f_{K a K a}\left(K_{a}\right)+E\left(G_{b}\right) \cdot f_{K b K b}\left(K_{b}\right)\right)}=\frac{d C}{d G_{a}}$ bzw. $\tilde{t}_{K} \cdot \frac{d E}{d G_{b}} \cdot \frac{f_{K b}\left(K_{b}\right)}{-\left(E\left(G_{a}\right) \cdot f_{K a K a}\left(K_{a}\right)+E\left(G_{b}\right) \cdot f_{K b K b}\left(K_{b}\right)\right)}=\frac{d C}{d G_{b}}$ nur eine eindeutige Lösung für $G_{i}$ besitzen. Bei der Reaktionsfunktion einer Leviathanregierung handelt es sich letztlich um die 1. Ableitung der Surplusfunktion nach $\mathrm{G}_{\mathrm{a}}$ bzw. $\mathrm{G}_{\mathrm{b}}$, die null entspricht:

$$
\frac{d S_{i}}{d G_{i}}=\tilde{t}_{K} \cdot \frac{\partial K_{i}}{\partial G_{i}}-\frac{d C}{d G_{i}}=0 \text {. }
$$

Da diese Gleichung die notwendige Bedingung für ein Maximum ist, gilt:

$$
\frac{d^{2} S_{i}}{d G_{i}^{2}}<0
$$

Die Reaktionsfunktion ist also streng monoton fallend und somit umkehrbar. ${ }^{1}$ Eine umkehrbare Funktion ist aber gerade dadurch definiert, dass sie nur eine Lösung für $\mathrm{G}_{i}$ besitzt. Somit hat die Reaktionsfunktion der Leviathanregierung nur eine eindeutige Lösung für $G_{i}$. 


\section{Anhang 10}

Um zu zeigen, dass das gefundene Nash-Gleichgewicht einmalig ist, wird von folgender Überlegung ausgegangen:

Die Reaktionsfunktionen besitzen - wie bereits in Abschnitt 4.2 dargelegt - nur eine eindeutige Lösung. Fraglich ist jedoch, ob die Funktionen mehrere Schnittpunkte haben können. Die Funktionen schneiden sich, wenn Gleichung (4.35) erfüllt ist. Als Funktion $\Omega$ in Abhängigkeit von $G_{i}$ wird folgende Funktion definiert:

$$
\Omega\left(G_{i}\right)=\frac{\left(\frac{d E}{d G_{i}}\right)}{\left(\frac{d C}{d G_{i}}\right)} \cdot f_{K i}\left(K_{i}\right) .
$$

Leitet man $\Omega$ nach $\mathrm{G}_{\mathrm{i}}$ ab, so erhält man:

$$
\begin{aligned}
\frac{d \Omega}{d G_{i}}= & \frac{\left(\frac{d^{2} E}{d G_{i}^{2}}\right)}{\left(\frac{d C}{d G_{i}}\right)} \cdot f_{K i}\left(K_{i}\right)+\left(\frac{d E}{d G_{i}}\right) \cdot f_{K i}\left(K_{i}\right) \cdot(-1) \cdot\left(\frac{d C}{d G_{i}}\right)^{-2} \cdot\left(\frac{d^{2} C}{d G_{i}^{2}}\right) \\
& +\frac{\left(\frac{d E}{d G_{i}}\right)}{\left(\frac{d C}{d G_{i}}\right)} \cdot f_{K i K i}\left(K_{i}\right) \cdot\left(\frac{\partial K_{i}}{\partial G_{i}}\right) \\
\Leftrightarrow \frac{d \Omega}{d G_{i}} & =\frac{\left(\frac{d^{2} E}{d G_{i}^{2}}\right)}{\left(\frac{d C}{d G_{i}}\right)} \cdot f_{K i}\left(K_{i}\right)-\left(\frac{d E}{d G_{i}}\right) \cdot f_{K i}\left(K_{i}\right) \cdot \frac{\left(\frac{d^{2} C}{d G_{i}^{2}}\right)}{\left(\frac{d C}{d G_{i}}\right)^{2}} \\
& +\frac{\left(\frac{d E}{d G_{i}}\right)}{\left(\frac{d C}{d G_{i}}\right)} \cdot f_{K i K i}\left(K_{i}\right) \cdot\left(\frac{\partial K_{i}}{\partial G_{i}}\right)<0 .
\end{aligned}
$$


$\Omega$ ist also eine streng monoton fallende Funktion und besitzt somit eine Umkehrfunktion. 'Insofern kann $\Omega\left(G_{a}\right)=\Omega\left(G_{b}\right)$ (Gleichung (4.35)) nur gültig sein, wenn $G_{a}=G_{b}$ vorliegt. Es handelt sich hierbei um die einzig mögliche Lösung. Das Nash-Gleichgewicht ist somit einmalig.

'Vgl. Chiang (1984, S. 171 f.). 


\section{Anhang 11}

Die Regierungen maximieren beide gleichzeitig ihren Surplus, wenn in beiden Regionen

$\frac{\left(\left.\frac{d E}{d G_{i}}\right|_{G_{i}=G_{i}^{\otimes}}\right)}{\left(\left.\frac{d C}{d G_{i}}\right|_{G_{i}=G_{i}^{\otimes}}\right) \cdot E\left(G_{i}^{\otimes}\right)}=\frac{(-2)}{\widetilde{t}_{K}} \cdot \frac{f_{\bar{K} \frac{\bar{K}}{2}}\left(\frac{\bar{K}}{2}\right)}{f_{\frac{\bar{K}}{2}\left(\frac{\bar{K}}{2}\right)}}$

bzw.

$\frac{\tilde{t}_{K} \cdot\left(\left.\frac{d E}{d G_{i}}\right|_{G_{i}=G_{i}^{\otimes}}\right)}{\left(\left.\frac{d C}{d G_{i}}\right|_{G_{i}=G_{i}^{\otimes}}\right) \cdot E\left(G_{i}^{\otimes}\right)}=(-2) \cdot \frac{f_{\bar{K} \bar{K}}\left(\frac{\bar{K}}{2}\right)}{f_{\frac{\bar{K}}{2}}\left(\frac{\bar{K}}{2}\right)}$

gilt.

Leitet man die letzte Gleichung nach $\tilde{t}_{K}$ ab, so gelangt man zu:

$\frac{\left(\left.\frac{d E}{d G_{i}}\right|_{G_{i}=G_{i}^{\otimes}}\right)}{\left(\left.\frac{d C}{d G_{i}}\right|_{G_{i}=G_{i}^{\otimes}}\right) \cdot E\left(G_{i}^{\otimes}\right)}+\frac{\tilde{t}_{K} \cdot\left(\left.\frac{d^{2} E}{d G_{i}^{2}}\right|_{G_{i}=G_{i}^{\otimes}}\right) \cdot\left(\frac{d G_{i}^{\otimes}}{d \widetilde{t}_{K}}\right)}{\left(\left.\frac{d C}{d G_{i}}\right|_{G_{i}=G_{i}^{\otimes}}\right) \cdot E\left(G_{i}^{\otimes}\right)}$

$\frac{\tilde{t}_{K} \cdot\left(\left.\frac{d E}{d G_{i}}\right|_{G_{i}=G_{i}^{\otimes}}\right) \cdot\left(\left.\frac{d^{2} C}{d G_{i}^{2}}\right|_{G_{i}=G_{i}^{\otimes}}\right) \cdot \frac{d G_{i}^{\otimes}}{d \widetilde{t}_{K}} \tilde{t}_{K} \cdot\left(\left.\frac{d E}{d G_{i}}\right|_{G_{i}=G_{i}^{\otimes}}\right)^{2} \cdot \frac{d G_{i}^{\otimes}}{d \widetilde{t}_{K}}}{\left(\left.\frac{d C}{d G_{i}}\right|_{G_{i}=G_{i}^{\otimes}}\right)^{2} \cdot E\left(G_{i}^{\otimes}\right)}=0$ 
$\Leftrightarrow \frac{\left(\left.\frac{d E}{d G_{i}}\right|_{G_{i}=G_{i}^{\otimes}}\right)}{\left(\left.\frac{d C}{d G_{i}}\right|_{G_{i}=G_{i}^{\otimes}}\right) \cdot E\left(G_{i}^{\otimes}\right)}=\frac{\tilde{t}_{K} \cdot\left(\left.\frac{d^{2} E}{d G_{i}^{2}}\right|_{G_{i}=G_{i}^{\otimes}}\right) \cdot \frac{d G_{i}^{\otimes}}{d \widetilde{t}_{K}}}{\left(\left.\frac{d C}{d G_{i}}\right|_{G_{i}=G_{i}^{\otimes}}\right) \cdot E\left(G_{i}^{\otimes}\right)}+$

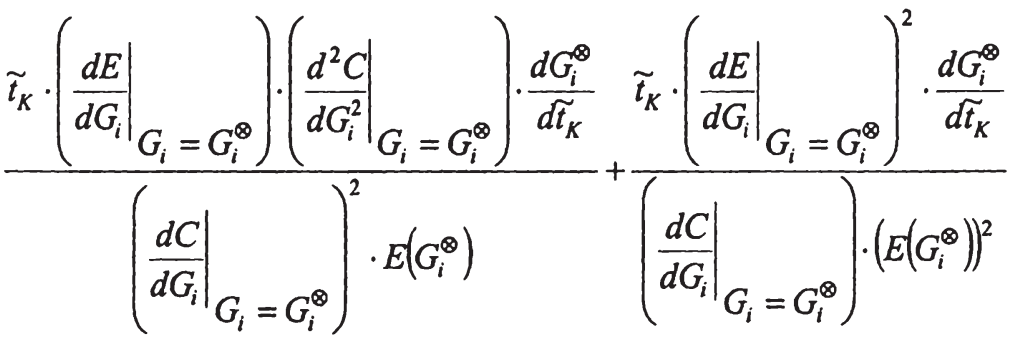

$\Leftrightarrow \frac{\left(\left.\frac{d E}{d G_{i}}\right|_{G_{i}=G_{i}^{\otimes}}\right)}{\left(\left.\frac{d C}{d G_{i}}\right|_{G_{i}=G_{i}^{\otimes}}\right) \cdot E\left(G_{i}^{\otimes}\right)}=-\frac{\tilde{t}_{K} \cdot\left(\left.\frac{d^{2} E}{d G_{i}^{2}}\right|_{G_{i}=G_{i}^{\otimes}}\right.}{\left(\left.\frac{d C}{d G_{i}}\right|_{G_{i}=G_{i}^{\otimes}}\right) \cdot E\left(G_{i}^{\otimes}\right)}+$

$\left.\frac{\tilde{t}_{K} \cdot\left(\left.\frac{d E}{d G_{i}}\right|_{G_{i}=G_{i}^{\otimes}}\right) \cdot\left(\left.\frac{d^{2} C}{d G_{i}^{2}}\right|_{G_{i}=G_{i}^{\otimes}}\right)}{\left(\left.\frac{d C}{d G_{i}}\right|_{G_{i}=G_{i}^{\otimes}}\right)^{2} \cdot E\left(G_{i}^{\otimes}\right)}+\frac{\tilde{t_{K}} \cdot\left(\left.\frac{d E}{d G_{i}}\right|_{G_{i}=G_{i}^{\otimes}}\right)^{2}}{\left(\left.\frac{d C}{d G_{i}}\right|_{G_{i}=G_{i}^{\otimes}}\right) \cdot\left(E\left(G_{i}^{\otimes}\right)\right)^{2}}\right) \cdot \frac{d G_{i}^{\otimes}}{d \tilde{t}_{K}}$. 
Als Ergebnis erhält man folglich:

$\frac{d G_{i}^{\otimes}}{d \tilde{t}_{K}}=\left(\frac{\left.\left|\frac{d E}{d G_{i}}\right|_{G_{i}=G_{i}^{\otimes}}\right)}{\left.\left|\frac{d C}{d G_{i}}\right|_{G_{i}=G_{i}^{\otimes}}\right) \cdot E\left(G_{i}^{\otimes}\right)} \cdot\left(-\frac{\left.\tilde{t}_{K} \cdot\left|\frac{d^{2} E}{d G_{i}^{2}}\right|_{G_{i}=G_{i}^{\otimes}}\right)}{\left.\left|\frac{d C}{d G_{i}}\right|_{G_{i}=G_{i}^{\otimes}}\right) \cdot E\left(G_{i}^{\otimes}\right)}+\right.\right.$

$\left.\frac{\tilde{t}_{K} \cdot\left(\left.\frac{d E}{d G_{i}}\right|_{G_{i}=G_{i}^{\otimes}}\right) \cdot\left(\left.\frac{d^{2} C}{d G_{i}^{2}}\right|_{G_{i}=G_{i}^{\otimes}}\right)}{\left(\left.\frac{d C}{d G_{i}}\right|_{G_{i}=G_{i}^{\otimes}}\right)^{2} \cdot E\left(G_{i}^{\otimes}\right)}+\frac{\tilde{t_{K}} \cdot\left(\left.\frac{d E}{d G_{i}}\right|_{G_{i}=G_{i}^{\otimes}}\right)^{2}}{\left(\left.\frac{d C}{d G_{i}}\right|_{G_{i}=G_{i}^{\otimes}}\right) \cdot\left(E\left(G_{i}^{\otimes}\right)\right)^{2}}\right)^{-1}$. 


\section{Literatur}

- Apolte, Thomas (2001). How tame will Leviathan become in institutional competition? In: Public Choice, 107, S. 359-381.

- Apolte, Thomas (1999). Chancen und Risiken nationaler Wirtschaftspolitik bei hoher Kapitalmobilität, in: Apolte, Thomas / Caspers, Rolf / Welfens, Paul J.J. (Hrsg.), Standortwettbewerb, wirtschaftliche Rationalität und internationale Ordnungspolitik, S. 21-44.

- Arnold, Volker / Fuest, Clemens (1999). Fiscal Competition and the Efficiency of Public Input Provision with Asymmetric Jurisdictions, in: Finanzarchiv, 56, S. 165-173.

- Atkinson, Anthony B. / Stiglitz, Joseph E. (1980). Lectures on Public Economics, Maidenhead.

- Beckmann, Klaus / Lackner, Elisabeth (1998). Vom Leviathan und von optimalen Steuern, Volkswirtschaftliche Reihe, V-6-98, Passau.

- Bewley, Truman F. (1981). A Critique of Tiebout's Theory of Local Public Expenditures, in: Econometrica, 49, S. 713-740.

- Bjorvatn, Kjetil / Schjelderup, Guttorm (2002). Tax Competition and International Public Goods, in: International Tax and Public Finance, 9, S. 111-120.

- Blankart, Charles B. (2001). Öffentliche Finanzen in der Demokratie, München.

- Boadway, Robin W. / Wildasin, David E. (1984). Public Sector Economics, Boston.

- Boskin, Michael J. (1973). Local Government Tax and Product Competition and the Optimal Provision of Public Goods, in: Journal of Political Economy, 81, S. 203-210.

- Braid, Ralph M. (1996). Symmetric Tax Competition with Multiple Jurisdictions in Each Metropolitan Area, in: The American Economic Review, 86, S. 1279-1290.

- Break, George F. (1967). Intergovernmental Fiscal Relations in the United States, Washington.

- Brennan, Geoffrey (1997). Public Choice and Taxation: Leviathan after Twenty Years, in: Krever, R. (Hrsg.), Tax Conversations, S. 87-105.

- Brennan, Geoffrey / Buchanan, James M. (1980). The Power to Tax, Cambridge. 
- Brueckner, Jan K. (1999). Fiscal Federalism and Capital Accumulation, in: Journal of Public Economic Theory, 1, S. 205-224.

- Brueckner, Jan K. (1983). Property Value Maximization and Public Sector Efficiency, in: Journal of Urban Economics, 14, S. 1-15.

- Brümmerhoff, Dieter (1996). Finanzwissenschaft, München.

- Buchanan, James M. / Lee, Dwight R. (1994). On a Fiscal Constitution for the European Union, in: Journal des Economistes et des Etudes Humaines, 5, S. 219-232.

- Bucovetsky, Sam (1991). Asymmetric Tax Competition, in: Journal of Urban Economics, 30, S. 167-181.

- Bucovetsky, S. / Marchand, M. / Pestieau, P. (1998). Tax Competition and Revelation of Preferences for Public Expenditure, in: Journal of Urban Economics, 44, S. 367-390.

- Bucovetsky, Sam / Wilson, John Douglas (1991). Tax competition with two tax instruments, in: Regional Science and Urban Economics, 21, S. 333-350.

- Burbidge, John B. / Myers, Gordon M. (1994). Population mobility and capital tax competition, in: Regional Science and Urban Economics, 24, S. 441459.

- Chiang, Alpha C. (1984). Fundamental Methods of Mathematical Economics, London.

- Colombier, Carsten (2001). Spezifikation einer Produktionsfunktion mit einem nichtrival nutzbaren Produktionsfaktor, Berlin.

- Colombier, Carsten / Pickhardt, Michael (1998). Die Bedeutung der Gutseigenschaften für die Modellierung von Zwischenprodukten und Konsumgütern, FISK Diskussionsbeitrag, Wuppertal.

- Cournot, A. A. (1838). Recherches sur les principes mathématiques de la théorie des richesses, Paris.

- DePater, James A. / Myers, Gordon M. (1994). Strategic Capital Tax Competition: A Pecuniary Externality and a Corrective Device, in: Journal of Urban Economics, 36, S. 66-78.

- Deutscher Bundestag (2000). Entwurf eines Gesetzes zur Senkung der Steuersätze und zur Reform der Unternehmensbesteuerung (Steuersenkungsgesetz - StSenkG), Bundestagsdrucksache 14/2683 vom 15.02.2000.

- Ebert, Werner / Meyer, Steffen (1999). Die Anreizwirkungen des Finanzausgleichs, in: Wirtschaftsdienst, 79, S. 106-114. 
- Edwards, Jeremy / Keen, Michael (1996). Tax competition and Leviathan, in: European Economic Review, 40, S. 113-134.

- Eggert, Wolfgang (1999). Capital Tax Competition with Inefficient Government Spending, Zentrum für Finanzen und Ökonometrie, Konstanz

- Eggert, Wolfgang / Haufler, Andreas (1999). Capital taxation and production efficiency in an open economy, in: Economics Letters, 62, S. 85-90.

- Feehan, James P. (1989). Pareto-Efficiency with three Varieties of Public Input, in: Public Finance, S. 237-248.

- Feehan, James P. / Matsumoto, Mutsumi (2002). Distortionary Taxation and Optimal Public Spending on Productive Activities, in: Economic Inquiry, 40, S. 60-68.

- Flatters, Frank / Henderson, Vernon / Mieszkowski, Peter (1974). Public Goods, Efficiency, and Regional Fiscal Equalization, in: Journal of Public Economics, 3, S. 99-112.

- Fleißig, Eva (2000). Die Harmonisierung der Zinsbesteuerung in der EU, Lohmar / Köln.

- Flochel, Laurent / Madies, Thierry (2002). Interjurisdictional Tax Competition in a Federal System of Overlapping Revenue Maximizing Governments, in: International Tax and Public Finance, 9, S. 121-141.

- Frey, Bruno S. / Eichenberger, Reiner (1996). To harmonize or to compete? That's not the question, in: Journal of Public Economics, 60, S. 335-349.

- Friedman, James W. (1983). Oligopoly theory, Cambridge.

- Friedman, James W. (1977). Oligopoly and the theory of games, Amsterdam.

- Fuest, Clemens (2000). The political economy of tax coordination as a bargaining game between bureaucrats and politicians, in: Public Choice, 103, S. 357-382.

- Fuest, Clemens (1997). Internationale Koordination der Fiskalpolitik: Wohlfahrtsökonomische versus Politökonomische Sicht, in: Jahrbuch für Wirtschaftswissenschaften, 48, S. 174-192.

- Fuest, Clemens (1995). Interjurisdictional Competition and Public Expenditure: Is Tax Co-ordination Counterproductive? In: Finanzarchiv, 52, S. 478496.

- Gordon, Roger H. (1983). An Optimal Taxation Approach to Fiscal Federalism, in: Quarterly Journal of Economics, XCVIII, S. 567-586. 
- Graf, Gerhard (1999). Grundlagen der Finanzwissenschaft, Heidelberg.

- Hange, Ulrich / Wellisch, Dietmar (1998). The Benefit of Fiscal Decentralization, in: Finanzarchiv, 55, S. 315-327.

- Heap, Shaun P. Hargreaves / Varoufakis, Yanis (1995). Game theory, London and New York.

- Hensberg, Claudia (2002). Capital Market in Intergovernmental Tax Competition, FISK Diskussionsbeitrag, Wuppertal.

- Hensberg, Claudia (2000). Internationale Studie über die Bedeutung der Unternehmenbesteuerung für Investitionen, Frankfurt am Main.

- Hercowitz, Zvi / Pines, David (1991). Migration with fiscal externalities, in: Journal of Public Economics, 46, S. 163-180.

- Holler, Manfred J. / Illing, Gerhard (1991). Einführung in die Spieltheorie, Berlin.

- Homburg, Stefan (1999). Competition and Co-ordination in International Capital Income Taxation, in: Finanzarchiv, 56, S. 1-17.

- Hoyt, William H. (1999). Leviathan, local government expenditures, and capitalization, in: Regional Science and Urban Economics, 29, S. 155-171.

- Kanbur, Ravi / Keen, Michael (1993). Jeux Sans Frontières: Tax Competition and Tax Coordination When Countries Differ in Size, in: The American Economic Review, 83, S. 877-892.

- Kaizuka, Keimei (1965). Public Goods and Decentralization of Production, in: The Review of Economics and Statistics, 47, S. 118-120.

- Kamada, Koichiro (1989). Tax Harmonization from Leviathan's Point of View. Bachelor of Economics, University of Tokyo.

- Keen, Michael / Marchand, Maurice (1997). Fiscal competition and the pattern of public spending, Journal of Public Economics, 66, S. 33-53.

- Kollintzas, Tryphon / Philippopoulos, Apostolis / Vassilatos, Vanghelis (2000). Is Tax Policy Coordination Necessary? Discussion Paper No. 2348, Centre for Economic Policy Research, London.

- Krelove, R. (1993). The persistence and inefficiency of property tax finance of local public expenditures, in: Journal of Public Economics, 51, S. 415-435.

- Laussel, Didier / Le Breton, Michel (1998). Existence of Nash equilibria in fiscal competition models, in: Regional Science and Urban Economics, 28, S. 283-296. 
- Mans, A. (1996). Steuerpolitik in der Europäischen Union zwischen Wettbewerb und Harmonisierung, in: Deutsches Institut für Wirtschaftsforschung, Vierteljahresheft 3/96, S. 331-353.

- McLure, Charles E. (1986). Tax Competition: Is What's Good For The Private Goose Also Good For The Public Gander, in: National Tax Journal, 39, S. 341-348.

- Michael, Michael S. (1995). Foreign Capital Inflow with Public Input Production, in: Journal of Economic Integration, 10, S. 72-86.

- Mieszkowski, Peter / Zodrow, George R. (1989). Taxation and the Tiebout Model: The Differential Effects of Head Taxes, Taxes on Land Rents, and Property Taxes, in: Journal of Economic Literature, XXVII, S. 1098-1146.

- Mintz, Jack / Tulkens, Henry (1986). Commodity Tax Competition between Member States of a Federation: Equilibrium and Efficiency, in: Journal of Public Economics, 29, S. 133-172.

- Musgrave, Richard A. (1971). Economics of Fiscal Federalism, in: Nebraska Journal of Economics and Business, 10, S. 3-13.

- Myers, Gordon M. (1990). Optimality, Free Mobility, and the Regional Authority in a Federation, in: Journal of Public Economics, 43, S. 107-121.

- Nash, John (1951). Non-Cooperative Games, in: Annals of Mathematics, 54, S. 286-295.

- Nechyba, Thomas J. (1997). Local Property and State Income Taxes: The Role of Interjurisdictional Competition and Collusion, in: Journal of Political Economy, 105, S. 351-384.

- Niskanen, William A. (1997). Autocratic, Democratic, and Optimal Government, in: Economic Inquiry, XXXV, S. 464-479.

- Nowotny, Ewald (1999). Der öffentliche Sektor, Berlin.

- Oates, Wallace E. (1990). Decentralization of the Public Sector: An Overview, in: Bennett, R. (Hrsg.), Decentralization, Local Governments, and Markets, Oxford, S. 43-58.

- Oates, Wallace E. (1977). An Economist's Perspective on Fiscal Federalism, in: Oates, Wallace E. (Hrsg.), The Political Economy of Fiscal Federalism, Lexington, S. 3-20.

- Oates, Wallace E. (1972). Fiscal Federalism, New York. 
- Oates, Wallace E. / Schwab, Robert M. (1988). Economic Competition among Jurisdictions: Efficiency Enhancing or Distortion Inducing? In: Journal of Public Economics, 35, S. 333-354.

- Peffekoven, Rolf (1994). Reform des Finanzausgleichs - eine vertane Chance, in: Finanzarchiv, 51, S. 281-311.

- Pitlik, Hans (1998). Steuerwettbewerb oder Steuerharmonisierung in der WWU? In: Caesar, Rolf / Scharrer, Hans-Eckart (Hrsg.). Die Europäische Wirtschafts- und Währungsunion: regionale und globale Herausforderungen, Bonn.

- Rauscher, Michael (1998). Leviathan and Competition among Jurisdictions: The Case of Benefit Taxation, in: Journal of Urban Economics, 44, S. 59-67.

- Razin, Assaf / Sadka, Efraim (1991). International tax competition and gains from tax harmonization, in: Economics Letters, 37, S. 69-76.

- Richter, Wolfram F. (1994). The efficient allocation of local public factors in Tiebout's tradition, in: Regional Science and Urban Economics, 24, S. 323340.

- Richter, Wolfram F. / Seitz, Helmut / Wiegard, Wolfgang (1996). Steuern und unternehmensbezogene Staatsausgaben als Standortfaktoren, in: Siebert, Horst (Hrsg.), Steuerpolitik und Standortqualität, Tübingen, S. 13-47.

- Richter, Wolfram F. / Wellisch, Dietmar (1996).The provision of local public goods and factors in the presence of firm and household mobility, in: Journal of Public Economics, 60, S. 73-93.

- Richter, Wolfram F. / Wellisch, Dietmar (1993). Allokative Theorie eines interregionalen Finanzausgleichs bei unvollständiger Landrentenabsorption, in: Finanzarchiv, 50, S. 433-457.

- Rieck, Christian (1993). Spieltheorie: Einführung für Wirtschafts- und Sozialwissenschaftler, Wiesbaden.

- Roloff, Otto (2001). Der eigennützige Staat in der Konfliktgesellschaft, Marburg.

- Roloff, Otto (1999). Fiskalische Standortkonkurrenz in Europa, in: FriedrichEbert-Stiftung (Hrsg.), Steuerreformen in Deutschland im europäischen Kontext, Bonn, S. 47-55.

- Roloff, Otto (1997). A Surplus from Investment in Infrastructure. An Incentive for Intergovernmental Competition, FISK Diskussionsbeitrag, Wuppertal. 
- Roloff, Otto / Brander, Sibylle / Barens, Ingo / Wesselbaum, Claudia (1994). Direktinvestitionen und internationale Steuerkonkurrenz, Frankfurt am Main.

- Samuelson, Paul A. (1954). The Pure Theory of Public Expenditure, in: Review of Economics and Statistics, 36, S. 387-389.

- Sapusek, A. (1997). Ökonomische und juristische Analyse der Steuerharmonisierung in der Europäischen Union, Frankfurt/M.

- Sinn, Hans-Werner (1997). Das Selektionsprinzip und der Systemwettbewerb, in: Alois Oberhauser (Hrsg.), Fiskalföderalismus in Europa, Berlin, S. 9-60.

- Sinn, Hans-Werner (1990). Tax Harmonization and Tax Competition in Europe, in: European Economic Review, 34, S. 489-504.

- Sinn, Stefan (1992). The Taming of Leviathan, in: Constitutional Political Economy, 3, S. 177-196.

- Tausch, Cornelia (1998). Non-Identical Regions, Congestible Public Factors, and Fiscal Competition, in: Finanzarchiv, 55, S. 328-342.

- Tiebout, Charles M. (1956). A Pure Theory of Local Expenditures, in: Journal of Political Economy, S. 416-424.

- Wellisch, Dietmar (2000). Theory of Public Finance in a Federal State, Cambridge.

- Wellisch, Dietmar (1993). On the decentralized provision of public goods with spillovers in the presence of household mobility, in: Regional Science and Urban Economics, 23, S. 667-679.

- Wiegard, Wolfgang (1980). Distortionary Taxation in a Federal Economy, in: Zeitschrift für Nationalökonomie, 40, S. 183-206.

- Wildasin, David E. (1991). Some rudimetary 'duopolity' theory, in: Regional Science and Urban Economics, 21, S. 393-421.

- Wildasin, David E. (1989). Interjurisdictional Capital Mobility: Fiscal Externality and a Corrective Subsidy, in: Journal of Urban Economics, 25, S. 193 212.

- Wildasin, David E. (1988). Nash Equilibria in Models of Fiscal Competition, in: Journal of Public Economics, 35, S. 229-240.

- Wildavsky, Aaron (1990). A Double Security: Federalism as Competition, in: Cato Journal, 10, S. 39-58. 
- Wilson, John Douglas (1991). Tax competition with interregional differences in factor endowments, in: Regional Science and Urban Economics, 21, S. 423-451.

- Wilson, John Douglas (1986). A Theory of Interregional Tax Competition, in: Journal of Urban Economics, 19, S. 296-315.

- Wrede, Matthias (1999). Tragedy of the fiscal common?: Fiscal stock externalities in a Leviathan model of federalism, in: Public Choice, 101, S. 177193.

- Wrede, Matthias (1998). Household mobility and the moderate leviathan: Efficiency and decentralization, in: Regional Science and Urban Economics, 28, S. 315-328.

- Yang, Yong (1996). Tax competition under the threat of capital flight, in: Economics Letters, 53, S. 323-329.

- Zodrow, George R. / Mieszkowski, Peter (1986). Pigou, Tiebout, Property Taxation, and the Underprovision of Local Public Goods, in: Journal of Urban Economics, 19, S. 356-370. 


\section{FINANZWISSENSCHAFTLICHE SCHRIFTEN}

Band 1 Werner Steden: Finanzpolitik und Einkommensverteilung. Ein Wachstums- und Konjunkturmodell der Bundesrepublik Deutschland. 1979.

Band 2 Rainer Hagemann: Kommunale Finanzplanung im föderativen Staat. 1976.

Band 3 Klaus Scherer: Maßstäbe zur Beurteilung von konjunkturellen Wirkungen des öffentlichen Haushalts. 1977.

Band 4 Brita Steinbach: "Formula Flexibility" - Kritische Analyse und Vergleich mit diskretionärer Konjunkturpolitik. 1977.

Band 5 Hans-Georg Petersen: Personelle Einkommensbesteuerung und Inflation. Eine theoretisch-empirische Analyse der Lohn- und veranlagten Einkommenstever in der Bundesrepublik Deutschland. 1977.

Band 6 Friedemann Tetsch: Raumwirkungen des Finanzsystems der Bundesrepublik Deutschland. Eine Untersuchung der Auswirkungen der Finanzreform von 1969 aut die Einnahmenposition der untergeordneten Gebietskörperschaften und ihrer regionalpolitischen Zieladäquanz. 1978.

Band 7 Wilhelm Pfähler: Normative Theorie der fiskalischen Besteuerung. Ein methodologischer und theoretischer Beitrag zur Integration der normativen Besteuerungstheorie in der Wohlfahrtstheorie. 1978.

Band 8 Wolfgang Wiegard: Optimale Schattenpreise und Produktionsprogramme für öffentliche Unternehmen. Second-Best Modelle im finanzwirtschaftlichen Staatsbereich. 1978.

Band 9 Hans P. Fischer: Die Finanzierung des Umweltschutzes im Rahmen einer rationalen Umweltpolitik. 1978.

Band 10 Rainer Paulenz: Der Einsatz finanzpolitischer Instrumente in der Forschungs- und Entwicklungspolitik. 1978.

Band 11 Hans-Joachim Hauser: Verteilungswirkungen der Staatsverschuldung. Eine kreislauftheoretische Inzidenzbetrachtung. 1979.

Band 12 Gunnar Schwarting: Kommunale Investitionen. Theoretische und empirische Untersuchungen der Bestimmungsgründe kommunaler Investitionstätigkeit in NordrheinWestfalen 1965-1972. 1979.

Band 13 Hans-Joachim Conrad: Stadt-Umland-Wanderung und Finanzwirtschaft der Kernstädte. Amerikanische Erfahrungen, grundsätzliche Zusammenhänge und eine Fallstudie für das Ballungsgebiet Frankfurt am Main. 1980.

Band 14 Cay Folkers: Vermögensverteilung und staatliche Aktivität. Zur Theorie distributiver Prozesse im Interventionsstaat. 1981.

Band 15 Helmut Fischer: US-amerikanische Exportförderung durch die DISC-Gesetzgebung. 1981.

Band 16 Günter Ott: Einkommensumverteilungen in der gesetzlichen Krankenversicherung. Eine quantitative Analyse. 1981.

Band 17 Johann Hermann von Oehsen: Optimale Besteuerung. (Optimal Taxation). 1982.

Band 18 Richard Kössler: Sozialversicherungsprinzip und Staatszuschüsse in der gesetzlichen Rentenversicherung. 1982.

Band 19 Hinrich Steffen: Zum Handlungs- und Entscheidungsspielraum der kommunalen Investitionspolitik in der Bundesrepublik Deutschland. 1983.

Band 20 Manfred Scheuer: Wirkungen einer Auslandsverschuldung des Staates bei flexiblen Wechselkursen. 1983. 
Band 21 Christian Schiller: Staatsausgaben und crowding-out-Effekte. Zur Effizienz einer Finanzpolitik keynesianischer Provenienz. 1983.

Band 22 Hannelore Weck: Schattenwirtschaft: Eine Möglichkeit zur Einschränkung der öffentlichen Verwaltung? Eine ökonomische Analyse. 1983.

Band 23 Wolfgang Schmitt: Steuern als Mittel der Einkommenspolitik. Eine Ergänzung der Stabilitätspolitik? 1984.

Band 24 Wolfgang Laux: Erhöhung staatswirtschaftlicher Effizienz durch budgetäre Selbstbeschränkung? Zur Idee einer verfassungsmäßig verankerten Ausgabengrenze. 1984.

Band 25 Brita Steinbach-van der Veen: Steuerinzidenz. Methodologische Grundlagen und empirisch-statistische Probleme von Länderstudien. 1985.

Band 26 Albert Peters: Ökonomische Kriterien für eine Aufgabenverteilung in der Marktwirtschaft. Eine deskriptive und normative Betrachtung für den Allokationsbereich. 1985.

Band 27 Achim Zeidler: Möglichkeiten zur Fortsetzung der Gemeindefinanzreform. Eine theoretische und empirische Analyse. 1985.

Band 28 Peter Bartsch: Zur Theorie der längerfristigen Wirkungen 'expansiver' Fiskalpolitik. Eine dynamische Analyse unter besonderer Berücksichtigung der staatlichen Budgetbeschränkung und ausgewählter Möglichkeiten der öffentlichen Defizitfinanzierung. 1986.

Band 29 Konrad Beiwinkel: Wehrgerechtigkeit als finanzpolitisches Verteilungsproblem. Möglichkeiten einer Kompensation von Wehrungerechtigkeit durch monetäre Transfers. 1986.

Band 30 Wolfgang Kitterer: Effizienz- und Verteilungswirkungen des Steuersystems. 1986.

Band 31 Heinz Dieter Hessler: Theorie und Politik der Personalsteuern. Eine Kritik ihrer Einkommens- und Vermögensbegriffe mit Blick auf die Leistungsfähigkeitstheorie. 1994.

Band 32 Wolfgang Scherf: Die beschäftigungspolitische und fiskalische Problematik der Arbeitgeberbeiträge zur Rentenversicherung. Eine Auseinandersetzung mit der Kritik an der lohnbezogenen Beitragsbemessung. 1987.

Band 33 Andreas Mästle: Die Steuerunion. Probleme der Harmonisierung spezifischer Gütersteuern. 1987.

Band 34 Günter Ott: Internationale Verteilungswirkungen im Finanzausgleich der Europäischen Gemeinschaften. 1987.

Band 35 Heinz Haller: Zur Frage der zweckmäßigen Gestalt gemeindlicher Steuern. Ein Diskussionsbeitrag zur Gemeindesteuerreform. 1987.

Band 36 Thomas Kuhn: Schlüsselzuweisungen und fiskalische Ungleichheit. Eine theoretische Analyse der Verteilung von Schlüsselzuweisungen an Kommunen. 1988.

Band 37 Walter Hahn: Steuerpolitische Willensbildungsprozesse in der Europäischen Gemeinschaft. Das Beispiel der Umsatzssteuer-Harmonisierung. 1988.

Band 38 Ulrike Hardt: Kommunale Finanzkraft. Die Problematik einer objektiven Bestimmung kommunaler Einnahmemöglichkeiten in der gemeindlichen Haushaltsplanung und im kommunalen Finanzausgleich. 1988.

Band 39 Jochen Michaelis: Optimale Finanzpolitik im Modell überlappender Generationen. 1989.

Band 40 Bernd Raffelhüschen: Anreizwirkungen der sozialen Alterssicherung. Eine dynamische Simulationsanalyse. 1989.

Band 41 Berend Diekmann: Die Anleihe- und Darlehenstransaktionen der Europäischen Gemeinschaften. 1990.

Band 42 Helmut Kaiser: Konsumnachfrage, Arbeitsangebot und optimale Haushaltsbesteuerung. Theoretische Ergebnisse und mikroökonometrische Simulation für die Bundesrepublik Deutschland. 1990. 
Band 43 Rüdiger von Kleist: Das Gramm-Rudman-Hollings-Gesetz. Ein gescheiterter Versuch der Haushaltskonsolidierung. 1991.

Band 44 Rolf Hagedorn: Steuerhinterziehung und Finanzpolitik. Ein theoretischer Beitrag unter besonderer Berücksichtigung der Hinterziehung von Zinserträgen. 1991.

Band 45 Cornelia S. Behrens: Intertemporale Verteilungswirkungen in der gesetzlichen Krankenversicherung der Bundesrepublik Deutschland. 1991.

Band 46 Peter Saile: Ein ökonomischer Ansatz der Theorie der intermediären Finanzgewalten Die Kirchen als Parafisci. 1992.

Band 47 Peter Gottried: Die verdeckten Effizienzwirkungen der Umsatzsteuer. Eine empirische allgemeine Gleichgewichtsanalyse. 1992.

Band 48 Andreas Burger: Umweltorientierte Beschäftigungsprogramme. Eine Effizienzanalyse am Beispiel des "Sondervermögens Arbeit und Umwelt". 1992.

Band 49 Jeanette Malchow: Die Zuordnung verteilungspolitischer Kompetenzen in der Europäischen Gemeinschaft. Eine Untersuchung aufgrund einer Fortentwicklung der ökonomischen Theorie des Föderalismus. 1992.

Band 50 Barbara Seidel: Die Einbindung der Bundesrepublik Deutschland in die Europäischen Gemeinschaften als Problem des Finanzausgleichs. 1992.

Band 51 Ralph Wiechers: Markt und Macht im Rundfunk. Zur Stellung der öffentlich-rechtlichen Rundfunkanstalten im dualen Rundfunksystem der Bundesrepublik Deutschland. 1992.

Band 52 Klaus Eckhardt: Probleme einer Umweltpolitik mit Abgaben. 1993.

Band 53 Oliver Schwarzkopf: Die Problematik unterschiedlicher Körperschaftsteuersysteme innerhalb der EG. 1993.

Band 54 Thorsten Giersch: Bergson-Wohlfahrtsfunktion und normative Ökonomie. 1993.

Band 55 Li-Fang Chou: Selbstbeteiligung bei Arzneimitteln aus ordnungspolitischer Sicht. Das Beispiel der Bundesrepublik Deutschland. 1993.

Band 56 Harald Schlee: Einkommensteuerliche Behandlung von Transferzahlungen. Zur Neuordnung der Familienbesteuerung sowie der Besteuerung von Versicherungsleistungen und Sozialtransfers. 1994.

Band 57 Alexander Spermann: Kommunales Krisenmanagement. Reaktionen baden-württembergischer Stadtkreise auf steigende Sozialhilfekosten und Einnahmenausfälle (198092). 1993.

Band 58 Otto Roloff / Sibylle Brander / Ingo Barens / Claudia Wesselbaum: Direktinvestitionen und internationale Steuerkonkurrenz. 1994.

Band 59 Claudia Wesselbaum-Neugebauer: Internationale Steuerbelastungsvergleiche. 1994.

Band 60 Stephanie Miera: Kommunales Finanzsystem und Bevölkerungsentwicklung. Eine Analyse des kommunalen Finanzsystems vor dem Hintergrund der sich abzeichnenden Bevölkerungsentwicklung am Beispiel Niedersachsens unter besonderer Berücksichtigung des Landkreises Wolfenbüttel und seiner Gemeinden. 1994.

Band 61 Wolfgang Schert: Die Bedeutung des kaldorianischen Verteilungsmechanismus für die gesamtwirtschaftlichen Wirkungen der staatlichen Neuverschuldung. 1994.

Band 62 Rainer Volk: Vergleich der Vergünstigungseffekte der verschiedenen investitionsfördernden Maßnahmen. 1994.

Band 63 Hans-Georg Napp: Kommunale Finanzautonomie und ihre Bedeutung für eine effiziente lokale Finanzwirtschaft. 1994. 2., unveränderte Auflage 1994.

Band 64 Bernd Rahmann / Uwe Steinborn / Günter Vornholz: Empirische Analyse der Autonomie lokaler Finanzwirtschaften in der Europãischen Gemeinschaft. 1994. 
Band 65 Carsten Kühl: Strategien zur Finanzierung der Altlastensanierung. 1994.

Band 66 Stephan Boll: Intergenerationale Umverteilungswirkungen der Fiskalpolitik in der Bundesrepublik Deutschland. Ein Ansatz mit Hilfe des Generational Accounting. 1994.

Band 67 Karl Justus Bernhard Neumärker: Finanzverfassung und Staatsgewalt in der Demokratie. Ein Beitrag zur konstitutionellen Finanztheorie. 1995.

Band 68 Christian Haslbeck: Zentrale versus dezentrale Internalisierung externer Effekte bei unvollständiger Information. 1995.

Band 69 Regina Müller: Horizontale oder vertikale Transfers zur Durchsetzung eines horizontalen Finanzausgleichs. 1995.

Band 70 Christian Hockenjos: Öffentliche Sportförderung in der Bundesrepublik Deutschland. Darstellung und finanztheoretische Analyse. 1995.

Band 71 Manfred Rosenstock: Die Kontrolle und Harmonisierung nationaler Beihilfen durch die Kommission der Europäischen Gemeinschaften. 1995.

Band 72 Christian Rüsch: Wohnungsbau- und Wohneigentumspolitik im Rahmen der Einkommensteuer. Eine Analyse unter steuersystematischen, verteilungspolitischen und fiskalischen Aspekten. 1996.

Band 73 Stephan Winters: Die kollektive Vorsorge für den Pflegefall im Alter. Eine Untersuchung am Beispiel der gesetzlichen Pflegeversicherung in den Niederlanden. 1996.

Band 74 Knut Blind: Allokationsineffizienzen auf Sicherheitsmärkten: Ursachen und Lösungsmöglichkeiten. Fallstudie: Informationssicherheit in Kommunikationssystemen. 1996.

Band 75 Barbara Petrick-Rump: Ökonomische Wirkungen von Steueramnestien. Untersuchung konkreter Enfahrungen ausgewählter Länder mit dem Einsatz von Steueramnestien anhand eines effizienten Steueramnestieprogramms. 1996.

Band 76 Georg Hirte: Effizienzwirkungen von Finanzausgleichsregelungen. Eine Empirische Allgemeine Gleichgewichtsanalyse für die Bundesrepublik Deutschland. 1996.

Band 77 Ulrike Kirchhoff: Die rheinland-pfälzischen Gemeinden im System des Finanzausgleichs. 1996.

Band 78 Kerstin Keil: Der soziale Mietwohnungsbau: Mängel und Alternativen. 1996.

Band 79 Bernhard Manzke: Kinderlastenausgleich versus verstärkte Einwanderung. Alternative Ansätze zur langfristigen Sicherung der Gesetzlichen Rentenversicherung. 1997.

Band 80 Hariolf M. Wenzler: Institutionenökonomik und öffentliche Finanzkontrolle. Eine Analyse am Beispiel der Europäischen Union. 1997.

Band 81 Joachim Nagel: Supply-Side Policy in den USA. Eine theoretische und empirische Analyse der angebotsorientierten Wirtschaftspolitik Reagans unter besonderer Berücksichtigung steuerlicher Aspekte. 1997.

Band 82 Heinz Lampert: Krise und Reform des Sozialstaates. 1997.

Band 83 Monika Hanswillemenke / Bernd Rahmann: Zwischen Reformen und Verantwortung für Vollbeschäftigung. Die Finanz- und Haushaltspolitik der sozial-liberalen Koalition von 1969 bis 1982. 1997.

Band 84 Berthold Fürst: Die Maastrichter Budgetkriterien im Konflikt mit der Verschuldungsautonomie der deutschen Gebietskörperschaften. 1997.

Band 85 Burkhard Pahnke: Einkommensorientierte Förderung des sozialen Mietwohnungsbaues. Bestandsaufnahme und Kritik. 1998.

Band 86 Judith Safford: Staatsverschuldung im Vereinigten Königreich. Die öffentliche Verschuldung unter der Konservativen Regierung von 1979-1994. Ursachen und Auswirkungen. 1998. 
Band 87 Ralf Oberheide: Die Bekämpfung der Steuerumgehung. 1998.

Band 88 Achim Truger: Die neue Finanzwissenschaft zwischen Realitätsferne und Irrelevanz der Annahmen. Eine methodologische Analyse potentieller Verteidigungsstrategien der neuen Finanzwissenschaft gegen den Vorwurf der Realitätsłerne ihres entscheidungstheoretischen Fundamentes. 1998.

Band 89 Karin Bickel: Familienbezogene Elemente im System der gesetzlichen Rentenversicherung. Unter besonderer Berücksichtigung von Ein-Eltern-Familien. 1999.

Band 90 Wolfgang Scherf: Schlüsselzuweisungen und Kreisumlage. Die Problematik der Finanzierung der Landkreise am Beispiel des kommunalen Finanzausgleichs von RheinlandPfalz. 1998.

Band 91 Sandra Ehmann: Familienpolitik in Frankreich und Deutschland - ein Vergleich. 1999.

Band 92 Hendrik Suermann: Einkommensteuerliche Behandlung von Währungsgewinnen und -verlusten. Eine finanzwissenschaftliche Analyse des Steuerrechts in den USA und in Deutschland. 1999.

Band 93 Rolf Bösinger: Die Neuordnung des bundesstaatlichen Finanzausgleichs 1995. Eine theoretische und empirische Analyse unter Berücksichtigung von allokationstheoretischen und polit-ökonomischen Gesichtspunkten. 1999.

Band 94 Ulrich Ermschel: Finanzwirtschattliche Konsequenzen beim Übergang auf das Ursprungslandprinzip im Europälschen Binnenmarkt. Eine Untersuchung am Beispiel des unvollkommenen oligopolistischen Neufahrzeugmarktes. 1999.

Band 95 Ute Hansen: Überwälzte Leistungen der Administration. Eine empirische und theoretische Analyse. 2000.

Band 96 Hans-Werner Seiler: Zur Durchsetzung der Einmalbesteuenung deutscher Körperschaftsgewinne. Strategien zur Vermeidung der im deutschen Körperschaftsteuersystem angelegten Benachteiligung ausländischer Anteilseigner. Eine finanzwissenschaftliche Analyse. 2000.

Band 97 Steffen Meyer: Zwischenstaatliche Finanzzuweisungen im zusammenwachsenden Europa. Zur Gestaltung eines Finanzausgleichs für die Europäische Union. 2000.

Band 98 Marion Hübner: Ökodumping? Umweltpolitik in internationalen Oligopolmärkten. 2000.

Band 99 Christhart Bork: Steuern, Transfers und private Haushalte. Eine mikroanalytische Simulationsstudie der Aufkommens- und Verteilungswirkungen. 2000.

Band 100 Norbert Eichler: Die Probleme des Gemeindefinanzausgleichs im Kooperativen Föderalismus. Eine ökonomische Analyse am Beispiel des Bundeslandes Nordrhein-Westfalen. 2000.

Band 101 Wolfgang Scherf: Der Ländertinanzausgleich in Deutschland. Ungelöste Probleme und Ansatzpunkte einer Reform. 2000.

Band 102 Stefan Dietrich Josten: Staatsverschuldung, intertemporale Allokation und Wirtschaftswachstum. Eine theoretische Analyse staatlicher Verschuldungspolitik in Modellen exogenen und endogenen Wachstums. 2000.

Band 103 Axel Breitbach: Steuerhinterziehung und Schattenwirtschaft aus gesamtwirtschaftlicher Sicht. 2000.

Band 104 Alexander Spermann: Negative Einkommensteuer, Lohnsubventionen und Langzeitarbeitslosigkeit. 2001.

Band 105 Michael Broer: Der kommunale Finanzausgleich in Hessen. Historische Darstellung und ökonomische Analyse unter besonderer Berücksichtigung der Schlüsselzuweisungen. 2001.

Band 106 Jan-Paul Ritscher: Der Einsatz von Finanzderivaten unter einer modemisierten Schuldenstrukturpolitik des Bundes. 2002. 
Band 107 Martin Gasche: Dynamische Fiskalpolitik. Makroökonomische Wirkungen der Fiskalpolitik in einem Real Business Cycle-Modell. 2003.

Band 108 Felix Brosius: Internationaler Steuenwettbewerb und Koordination der Steuersysteme. 2003.

Band 109 Claudia Hensberg: Eigennützige Regierungen im fiskalischen Wettbewerb um Kapital. 2003. 


\section{Internationale Mobilität von und internationaler fiskalischer Wettbewerb um Direktinvestitionen}

Frankfurt/M., Berlin, Bern, Bruxelles, New York, Oxford, Wien, 2002. XXI, 235 S., zahlr. Abb. und Tab.

Schriften zur Internationalen Entwicklungs- und Umweltforschung. Herausgegeben vom Zentrum für internationale Entwicklungs- und Umweltforschung der Justus-Liebig-Universität Gießen. Bd. 4 ISBN 3-631-39600-7 • br. € 40.40*

Die Arbeit konzentriert sich auf Voraussetzungen und Wirkungen des internationalen fiskalischen Wettbewerbs um Direktinvestitionen. Zunächst werden verschiedene Mobilitätsbarrieren identifiziert. Unter Verwendung neokeynesianischer Theorieelemente wird eine Begründung dafür geliefert, weshalb innerhalb von bestimmten Ländergruppen, die als "Mobilitätsclubs" bezeichnet werden können, die Mobilität von Direktinvestitionen besonders hoch ist. Die anschließende Analyse des internationalen fiskalischen Wettbewerbs mit Hilfe von Steuer- und Ausgabenwettbewerb zeigt, dass ein intensiver Fiskalwettbewerb nur innerhalb von Länderclubs mit hoher Mobilität von Direktinvestitionen auftritt. Die Wirkungen hängen von der gewählten Strategie des fiskalischen Wettbewerbs ab und unterscheiden sich für multinationale Unternehmen und Unternehmen mit lokal begrenzten Investitionsaktivitäten.

Aus dem Inhalt: Internationaler Kapitalsteuerwettbewerb - Internationale Kapitaltransfers und Direktinvestitionen in einer Welt mit vollkommener Kapitalmobilität · Regionale Verteilung von grenzüberschreitenden Investitionen · Hindernisse der internationalen Kapitalmobilität - Theoretische Herleitung von Hindernissen der internationalen Mobilität von Direktinvesti-tionen - Staatliche Einnahmen und Ausgaben im internationalen fiskalischen Wettbewerb . Internationaler fiskalischer Wettbewerb - Empirische Befunde zur Entwicklung von Unternehmensbesteuerung und Ausgaben in der Europäischen Union

Frankfurt/M - Berlin - Bern - Bruxelles - New York - Oxford - Wien

Auslieferung: Verlag Peter Lang AG

Moosstr. 1, $\mathrm{CH}-2542$ Pieterlen

Telefax 0041 (0) $32 / 3761727$

*inklusive der in Deutschland gültigen Mehrwertsteuer

Preisänderungen vorbehalten

Homepage http://wuww.peterlang.de 
Claudia Hensberg - 978-3-631-75213-5

Downloaded from PubFactory at 01/11/2019 07:02:00AM

via free access 